\title{
PLASTICITY OF INHIBITION IN OLD AGE: \\ DURABILITY, PRACTICE, AND TRANSFER EFFECTS \\ by
}

Andrea J. Wilkinson

M.A. Psychology, Ryerson University, 2009

B.A. (Hons) Psychology \& Gerontology, McMaster University, 2006

\author{
A dissertation \\ presented to Ryerson University \\ in partial fulfillment of the \\ requirements for the degree of \\ Doctor of Philosophy \\ in the Program of \\ Psychology
}

Toronto, Ontario, Canada, 2014

(C)Andrea J. Wilkinson 2014 


\section{Author's Declaration}

I hereby declare that I am the sole author of this dissertation. This is a true copy of the dissertation, including any required final revisions, as accepted by my examiners.

I authorize Ryerson University to lend this dissertation to other institutions or individuals for the purpose of scholarly research.

I further authorize Ryerson University to reproduce this dissertation by photocopying or by other means, in total or in part, at the request of other institutions or individuals for the purpose of scholarly research.

I understand that my dissertation may be made electronically available to the public. 
Plasticity of Inhibition in Old Age: Durability, Practice, and Transfer Effects

Doctor of Philosophy, Spring 2014

Andrea J. Wilkinson

Psychology, Ryerson University

Empirical research indicates age-related declines in three sub-functions of inhibition: access (keeping irrelevant information outside one's focus of attention), deletion (ridding working memory of no longer relevant information), and restraint (withholding automatic responses that are not appropriate for the task at hand). Although single-task inhibition training has been previously explored using a six-session Stroop task program, no research has been done to examine long-term durability of the practice gains or the impact of a multi-task approach to inhibition training in older adults. This dissertation fills these gaps in the literature with three studies. The first study evaluates the maintenance of Stroop training one and three years following initial training and finds evidence in support of long-term durability of single-task inhibition training in older adults. The remaining two studies explored the benefits of training all three sub-functions of inhibition in older adults. First, study 2 seeks to confirm the presence of age differences in all three sub-functions of inhibition - supporting a rationale for training these abilities in older adults. Last, study 3 examines the plasticity of all three subfunctions of inhibition in older adults across six retest practice sessions, and three levels of associated transfer: near-near (transfer to the tasks used at training, but with varying items), near (transfer to tasks that were not trained, but tap the same abilities as the training tasks), and far (transfer to tasks that were not trained and tap abilities different from those trained). The findings indicate that older adults show retest practice gains in 
all three sub-functions of inhibition. Furthermore, strong evidence supports near-near transfer, while there is limited support for near transfer and no support for far transfer effects in older adults following three sub-functions of inhibition training. Taken together these studies contribute to the cognitive aging literature by evaluating several key features of plasticity in inhibition, including durability of training effects, retest practice and transfer effects. These findings have implications for the development of effective cognitive training programs in older adults. 


\section{Acknowledgements}

First and foremost, I would like to thank my academic supervisor, Dr. Lixia Yang, for her expertise, guidance, and support throughout the years. I have learned so much from working with you. I would also like to thank Dr. Todd Girard for his intellectual and thought provoking input at all stages of this dissertation. Also, thank you to all of the Research Assistants for their invaluable help with recruitment and data collection.

I would also like to thank my academic peers for being a great source of information, thoughtful discussion, and support throughout the years.

Last, but certainly not least, I would like to thank my family and friends for being my backbone and cheerleader during my entire graduate school career. I would like to give a special thank you to my partner, Sean, for always believing in me and being an unwavering source of companionship, comfort, and love. 


\section{Dedication}

For my mom and dad,

Words cannot describe how thankful I am for your encouragement and support.

"Education is the most powerful weapon which you can use to change the world"

-Nelson Mandela 


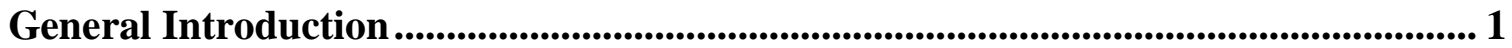

Inhibition: Definitions and Models .................................................................... 3

The primary model: Hasher and colleagues...................................................... 3

Supplementary models of inhibition................................................................. 4

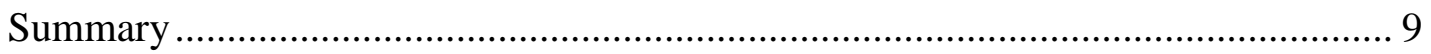

Age-related Deficits in Inhibition and the Associated Neural Correlates.................. 9

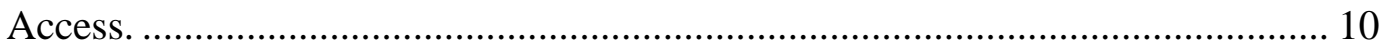

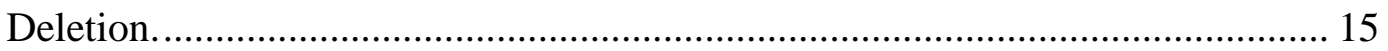

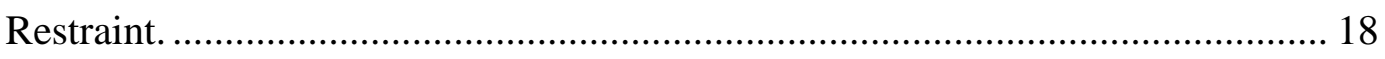

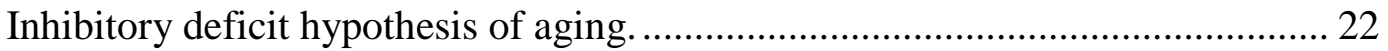

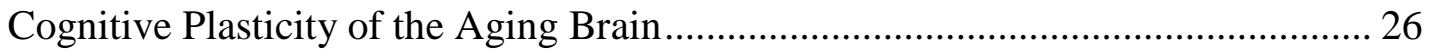

Plasticity of executive functions.................................................................. 27

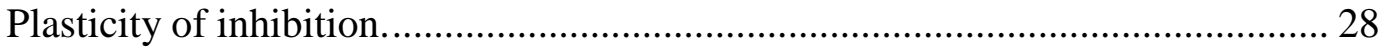

Long-term maintenance of training gains...................................................... 29

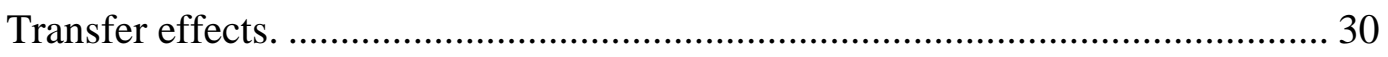

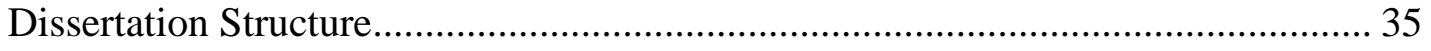

\section{Chapter 1. Long-term Maintenance of Retest Learning of Inhibition in Older}

Adults: 1-year and 3-year Follow-up ............................................................... 37

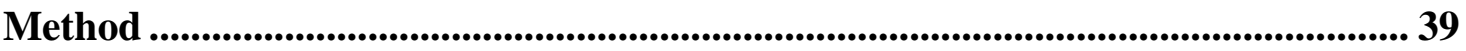

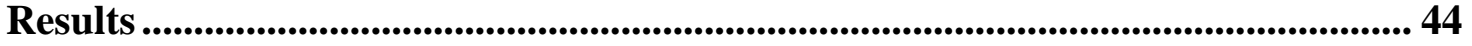

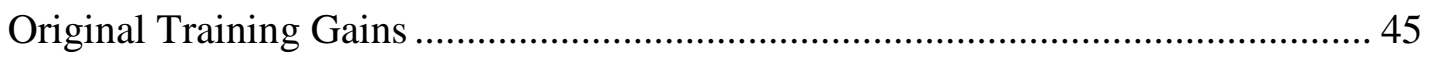

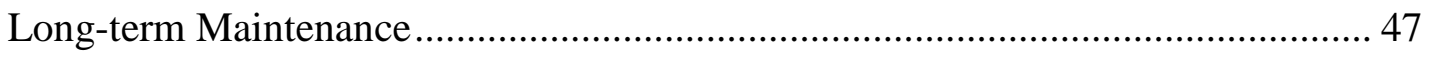

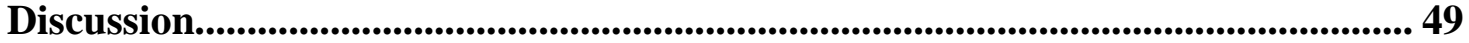




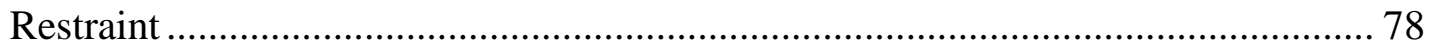

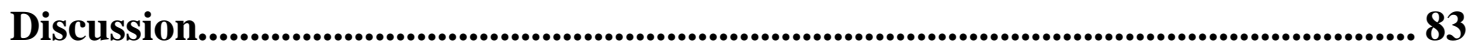

Chapter 3. Plasticity of Three Sub-functions of Inhibition in Older Adults: Retest

Practice and Transfer Effects ............................................................................................. 94

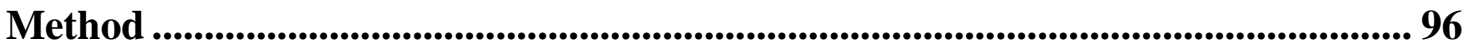

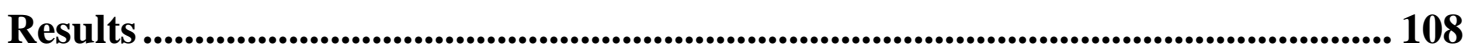

Data Processing (Local-Global, N-Back, and Go-No Go) ....................................... 109

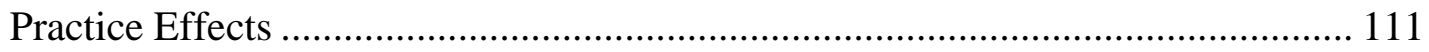

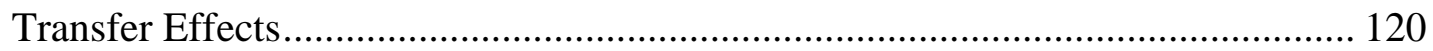

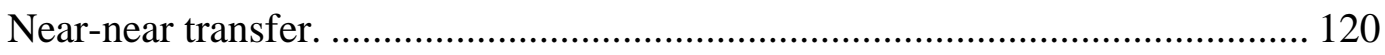

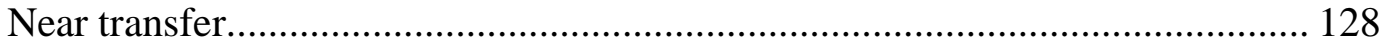

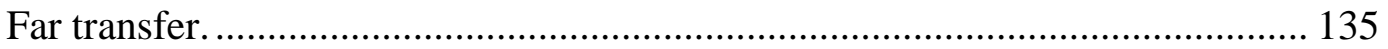

Discussion.................................................................................................................. 138

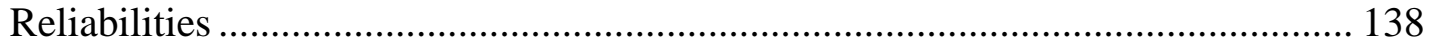

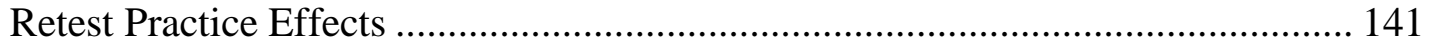

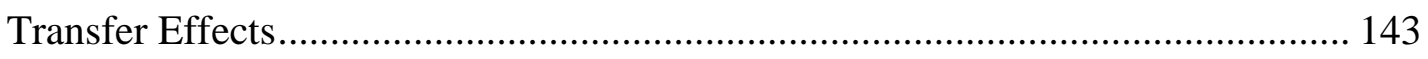

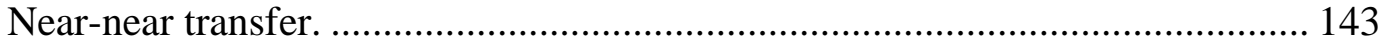

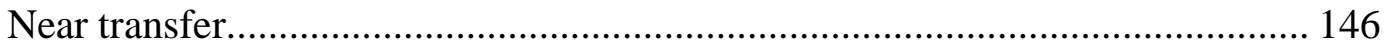

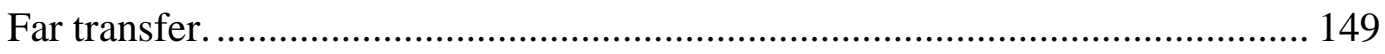

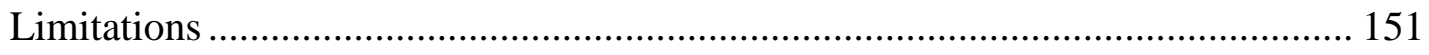

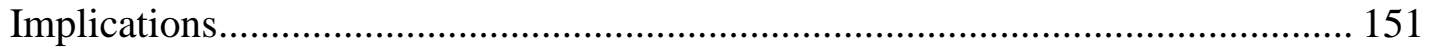

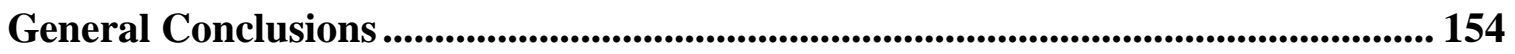

Appendices................................................................................................................................... 157

References ...................................................................................................................... 175 


\section{List of Tables}

\section{Chapter 1}

Table 1. Follow-up vs. No Follow-up Participant Demographic Characteristics and Baseline Cognitive Performance Assessed at Pretest

\section{Chapter 2}

Table 2. Participant Demographic Characteristics and Cognitive Performance

Table 3. Local-Global: Reaction Time, Accuracy and Interference Scores by Trial Type and Dimension Separately for Young and Older Adults

Table 4. Reading with Distraction: Passages Reading Speed, Multiple Choice Performance, and Proportion of Distractor Intrusions Separately for Young and Older Adults

Table 5. Directed Forgetting: Mean Reaction Time, Hit Rate and False Alarm Rate for Young and Older Adults

Table 6. Go - No Go: False Alarm Rate for Full and Reduced Sample Separately for Young and Older Adults

Table 7. Stroop: Accuracy, Reaction Time, and Interference Scores Separately for Young and Older Adults

\section{Chapter 3}

Table 8. Demographic Characteristics and Baseline Cognitive Performance Assessed at Pretest Separately for Training and No Training Control

Table 9. List of Tasks Administered at Pretest, Training, and Posttest Sessions

Table 10. Pretest - Posttest Performance in Reading with Distraction Separately for Training and No Training Control

Table 11. Pretest - Posttest Performance in Directed Forgetting Separately for Training 
and No Training Control

Table 12. Pretest - Posttest Performance in Stroop Separately for Training and No Training Control

Table 13. Pretest - Posttest Accuracy Performance in Corsi Block Separately for Training and No Training Control

Table 14. Pretest - Posttest Performance in Word List Recall Separately for Training and No Training Control

Table 15. Pretest - Posttest Performance in Letter Series and Digit Symbol Separately for Training and No Training Control

Table 16. Test-retest Reliabilities using Cronbach’s Alpha - Collapsed across Training and Control Groups 


\section{List of Figures}

\section{General Introduction}

Figure 1. Sample of Local-Global stimuli for target letters ' $A$ ' or 'D', with global dimension focus on the left and local dimension focus on the right.

\section{Chapter 1}

Figure 2. Mean RT Stroop interference scores (in ms) separately for those that completed the 1-year $(n=33)$ and 3-year follow-up sessions $(n=26)$ across the original six practice sessions. Error bars represent the standard error.

Figure 3. Mean RT Stroop interference scores (in ms) in week 1 and 2 of original training and the two follow-up sessions separately for those that completed the 1-year $(n=33)$ and 3-year follow-up sessions $(n=26)$. Error bars represent the standard error.

\section{Chapter 2}

Figure 4. Sample of Local-Global stimuli for target numbers ' 1 ' or '4', with global dimension focus on the left and local dimension focus on the right.

Figure 5. RT interference scores during local and global dimension focus separately for young and older adults. Error bars represent the standard error.

Figure 6. Proportion of accurate responses in N-Back across four conditions separately for young and older adults. Error bars represent the standard error.

Figure 7. Mean RT in Directed Forgetting for 'new' response to TBF and NEW words separately for young $(n=36)$ and older adults $(n=34)$. Error bars represent the standard error.

Figure 8. Proportion of accurate responses in Stroop for congruent, incongruent, and neutral trials separately for young and older adults. Error bars represent the standard error. 
Figure 9. Mean RT in Stroop for congruent, incongruent, and neutral trials separately for young and older adults. Error bars represent the standard error.

\section{Chapter 3}

Figure 10. Mean RT in Local-Global with local dimension focus for congruent, incongruent, and neutral trials across six practice sessions. Error bars represent the standard error.

Figure 11. Mean RT in Local-Global with global dimension focus for congruent, incongruent, and neutral trials across six practice sessions. Error bars represent the standard error.

Figure 12. RT interference scores in Local-Global separately for local and global dimension focus. Error bars represent the standard error.

Figure 13. Proportion of accurate responses in N-Back - collapsed across condition across six practice sessions. Error bars represent the standard error.

Figure 14. Proportion of accurate responses in N-Back for 1-Back, 2-Back and 3-Back conditions across six practice sessions. Error bars represent the standard error.

Figure 15. Proportion of false alarms in Go - No Go across six practice sessions. Error bars represent the standard error.

Figure 16. Proportion of false alarms in Go - No Go across six practice sessions with reduced sample $(n=14)$. Error bars represent the standard error.

Figure 17. Overall RT in Local-Global at pretest and posttest sessions separately for Training $(n=23)$ and Control $(n=24)$. Error bars represent the standard error. 
Figure 18. RT interference scores in Local-Global for local and global dimension focus at pretest and posttest sessions separately for Training $(n=23)$ and Control $(n=24)$. Error bars represent the standard error.

Figure 19. Proportion of accurate responses in N-Back for 0-Back, 1-Back, 2-Back, and 3-Back conditions at pretest and posttest sessions separately for Training and Control. Error bars represent the standard error.

Figure 20. Proportion of accurate responses in N-Back - collapsed across condition - at pretest and posttest sessions separately for Training and Control. Error bars represent the standard error.

Figure 21. Proportion of false alarms in Go - No Go at pretest and posttest sessions separately for Training and Control. Error bars represent the standard error.

Figure 22. Proportion of false alarms in Go - No Go at pretest and posttest sessions separately for Training $(n=18)$ and Control $(n=17)$ - with reduced samples. Error bars represent the standard error.

Figure 23. RT interference scores in Stroop at pretest and posttest sessions separately for Training and Control. Error bars represent the standard error. 


\section{List of Appendices}

Appendix A. REB Approval Form for Wilkinson and Yang (2012; Chapter 1)

Appendix B. REB Approval Form for the 'Age Differences in Three Sub-functions of Inhibition’ Study (Chapter 2)

Appendix C. REB Approval Form for the 'Plasticity of Three Sub-functions of Inhibition in Older Adults’ Study (Chapter 3)

Appendix D. Consent Agreement for the 1-year Follow-up Sample (Chapter 1)

Appendix E. Consent Agreement for the 3-year Follow-up Sample (Chapter 1)

Appendix F. Oral Debriefing for the 1 and 3-year Follow-up (Chapter 1)

Appendix G. Consent Agreement for the Young Adult Sample (Chapter 2)

Appendix H. Debriefing Form for the Young Adult Sample (Chapter 2)

Appendix I. Consent Agreement for the Training Group (Chapter 3)

Appendix J. Consent Agreement for the Control Group (Chapter 3)

Appendix K. Debriefing Form for the Training Group (Chapter 3)

Appendix L. Debriefing Form for the Control Group (Chapter 3) 


\section{GENERAL INTRODUCTION}

\section{General Introduction}

Executive functions are higher-level cognitive processes that guide and direct lower-level processing (Miyake et al., 2000). According to Miyake and colleagues (2000), there are three main executive functions: updating (i.e., the ability to refresh the contents of working memory), shifting (i.e., the ability to switch attention between different sets of task goals), and inhibition (i.e., the ability to suppress distraction). The focus of this dissertation is on inhibition, an executive function that is critical for successful living. Inhibition works to block out distracting information and control impulses, thus to keep thinking clear and attention sharply focused on the task at hand (Garavan, Ross, \& Stein, 1999; Hasher, Lustig, \& Zacks, 2007; Hasher, Zacks, \& May, 1999; Kipp Harnishfeger, 1995; Miyake et al., 2000). Attention and inhibition work together in that attention focuses on a target and inhibition works to suppress distracting information, allowing one to overcome interference and maintain attention on the target (Anderson, 2001) $)^{1}$. In other words, efficient attention requires not only the activation of the to-be-attended items, but also, importantly, the suppression of the to-be-ignored items (Kipp Harnishfeger, 1995; Houghton \& Tipper, 1994; Jonides, Badre, Curtis, ThompsonSchill, \& Smith, 2002). Inhibition is used regularly in everyday life - for example, blocking out surrounding conversations while trying to read the newspaper at a coffee shop or withholding the urge to check emails when trying to write a paper. "None of us enjoys accidentally walking to yesterday’s parking spot three blocks away from today’s, or misdialing an old telephone number after it has been changed. Furthermore, we all

\footnotetext{
${ }^{1}$ It is important to distinguish inhibition and interference. These two concepts work together - inhibition is the mechanism and interference is the phenomena. In particular, inhibition will be defined as the mechanism by which interference is reduced or resolved (Dempster, 1992; Friedman \& Miyake, 2004).
} 


\section{GENERAL INTRODUCTION}

have occasions when it is difficult to concentrate on a train of thought, because recent events or thoughts (pleasant or unpleasant) call our attention too powerfully” (Anderson, 2001, p.186). Even though we use inhibition on a regular basis, research consistently shows that this executive function declines as we age (e.g., Connelly, Hasher \& Zacks, 1991; Dobbs \& Rule, 1989; Gazzaley, Cooney, Rissman \& D’Esposito, 2005; Milham et al., 2002; West \& Alain, 2000; Zacks, Radvansky, \& Hasher, 1996). Of great importance, it has been suggested that deficits in inhibition underlie other cognitive declines observed in older adults (Hasher et al., 1999; Hasher et al., 2007). Given the critical role of inhibition in older adults' cognition and daily living, the main aims of the current dissertation are to assess the plasticity (i.e., improvement in ability using training; Baltes \& Lindenberger, 1988) of inhibition in older adults, as well as to examine whether this training-induced improvement is endurable across time and can be transferred to other tasks.

This general introduction contains three main sections. The first section focuses on the operational definition of inhibition, highlighting the model of inhibition proposed by Hasher and colleagues (e.g., 1999) that serves as the framework for the current dissertation. This section will also review various models in literature that parcel inhibition into several sub-functions and describe how these divisions can be mapped onto the three sub-function model of inhibition proposed by Hasher and colleagues. The second section provides an overview of the literature on age-related declines in each of the three sub-functions of inhibition as outlined by Hasher and colleagues. In addition, here, the inhibitory deficit hypothesis of aging will be reviewed to elucidate the link between poor inhibitory control and age-related declines in other cognitive domains, such 


\section{GENERAL INTRODUCTION}

as memory and processing speed. The third section will examine evidence demonstrating cognitive plasticity in older adults across various cognitive domains, including executive functions. In this section, the literature on the maintenance of training gains across time will be reviewed, as well as the transfer of improvement to other cognitive domains as shown in older adults. Based on the gaps in the literature identified in this section, three studies will be proposed and described for the dissertation.

\section{Inhibition: Definitions and Models}

In line with the majority of the literature (e.g., Dempster, 1993; English \& English, 1958; Friedman \& Miyake, 2004; Kipp Harnishfeger, 1995; Hasher et al., 1999; Hasher et al., 2007; Nigg, 2000; Wilson \& Kipp, 1998), herein inhibition will be considered not as a unitary construct, but as a cognitive ability that consists of several different sub-functions. Although there is a consensus in the literature about what inhibition means generally, there is little agreement about the terminology used to define its various sub-functions (Kipp Harnishfeger, 1995; Nigg, 2000). In the following section, an extensive overview of Hasher and colleagues' three sub-function model of inhibition (e.g., Hasher et al., 1999; Hasher et al., 2007) - a model that serves as the framework for this dissertation - will first be provided. Following this foundational definition, several competing multi-process models of inhibition will be outlined highlighting how each can be interpreted within Hasher's three sub-function framework to illustrate the generalizability of this model, as well as its parsimonious nature.

The primary model: Hasher and colleagues. According to Hasher and colleagues, there are three sub-functions of inhibition: access, deletion, and restraint each with a specific role at a different stage along the information processing stream that 


\section{GENERAL INTRODUCTION}

works to control the contents of working memory (Hasher et al., 1999; Hasher, Tonev, Lustig, \& Zacks, 2001; Hasher et al., 2007). The access sub-function of inhibition works at the entry point of working memory and functions to block irrelevant information from entering. This can be thought of as a gate to working memory, which opens to let relevant information in and closes to keep irrelevant information out (Badre, 2012). The deletion sub-function of inhibition, in contrast, acts on information only after it has entered the working memory storage space. Deletion works to remove or suppress information that is no longer relevant for the task at hand. The irrelevant information may have gained entry to working memory for several reasons: (a) it was previously or is potentially relevant to the task at hand given the instructions, or (b) it may have accidentally slipped into working memory due to a deficient access sub-function of inhibition. The last subfunction of inhibition, restraint, occurs at the output stage in the processing stream, when automatic, but inappropriate, responses are triggered, and must be withheld or suppressed given the task demands. The automatic responses can be either motoric (e.g., an automatic key press response) or cognitive (e.g., an automatic word reading response; Hasher et al., 1999; Hasher et al., 2001; Hasher et al., 2007). In sum, the three subfunctions of inhibition work to control the contents of working memory at different stages along the flow of an information processing stream: input (access), within working memory (deletion), and output (restraint).

Supplementary models of inhibition. The next section presents a series of alternative views of the construct of inhibition. One of the main goals of this section is to demonstrate how various alternative models of inhibition can be interpreted within Hasher and colleagues' three sub-function model. 


\section{GENERAL INTRODUCTION}

English \& English (1958). English and English (1958) defined inhibition as consisting of three functions. The first refers to the ability to stop a process (psychological or physiological) from continuing or stopping it from starting altogether. Similarly, the second type of inhibition refers to a psychological state in which mental action is curtailed. Both of these types of inhibition could be interpreted as being in line with Hasher's (1999, 2007) restraint sub-function of inhibition, as they refer to withholding or altering an overlearned response. The third type of inhibition defined by English and English (1958) refers to preventing an instinctual process from coming into consciousness. This type of inhibition overlaps with Hasher's access sub-function of inhibition given that it involves keeping something from conscious awareness. Other researchers have interpreted these definitions differently. For example, Macleod, Dodd, Sheard, Wilson, and Bibi (2003) termed the inhibitory categories outlined by English and English (1958) as suppression, restraint and blocking, respectively.

Dempster (1993). According to Dempster (1993), there are also three types of inhibitory sub-functions, each of which deals with a distinct type of interference. In particular, there are motoric, perceptual and linguistic sources of interference with which inhibition must deal. Motoric inhibition refers to the ability to withhold an automatic motor response that is inappropriate given the task goals. This definition loads perfectly onto Hasher's (1999, 2007) definition of restraint. Perceptual inhibition refers to the ability to keep internally or externally generated distracting information outside one’s focus of attention; thereby, helping to keep attention focused on the important, relevant bits of information. This type of inhibition fits in line most closely with Hasher's access sub-function of inhibition; but, can also be interpreted as deletion in that internally 


\section{GENERAL INTRODUCTION}

generated irrelevant pieces of information need to be suppressed. Last is linguistic inhibition where interference is specified to originate from language-related stimuli, such as in the Stroop task. In this task, participants are asked to respond to the ink colour of the stimuli. On incongruent trials, the colour word and ink colour is mismatched; therefore, the colour word is an interfering source of information that needs to be inhibited when trying to identify the ink colour. This, therefore, requires the use of linguistic inhibition. According to Dempster's (1993) interpretation of linguistic inhibition, it falls in line with Hasher's access sub-function of inhibition. However, others suggest that in order to successfully complete the Stroop task, withholding the automatic word reading response is required in order to be able to correctly identify the ink colour of the word (e.g., Hasher et al., 2007; Jonides et al., 2002; MacLeod \& MacDonald, 2000). This alternative view of the inhibitory function involved in the Stroop task falls in line with the restraint subfunction.

Kipp Harnishfeger (1995). Kipp Harnishfeger (1995) proposes that inhibition be defined by the process on which it is working, therefore resulting in two types of inhibition: behavioural and cognitive. Accordingly, behavioural inhibition is the act of suppressing motoric impulses, which is in line with Hasher’s (1999, 2007) restraint subfunction of inhibition. In contrast, cognitive inhibition is defined as control over mental processes that can be either conscious (overt) or subconscious (covert). The method used to control mental processes will dictate the type of inhibition that is involved. For example, if one is keeping unwanted thoughts outside of conscious awareness, this is in line with the access sub-function of inhibition. However, if one has an unwanted thought and then eliminates it from working memory, then one is utilizing the deletion sub- 


\section{GENERAL INTRODUCTION}

function of inhibition. Interestingly, Kipp Harnishfeger (1995) makes a distinction between controlled and automatic inhibition. In the former, irrelevant information is consciously identified and kept out of or removed from working memory, whereas, automatic inhibition occurs when irrelevant information is suppressed prior to conscious awareness - e.g., as in negative priming (also see Wilson \& Kipp, 1998).

Nigg (2000). Nigg (2000) proposes an eight level taxonomy of inhibition; however, here, the focus will only be on that which is most relevant to the current dissertation: executive inhibition. Within the executive inhibition domain, Nigg outlines four types of inhibition: (a) interference control (i.e., keeping conflicting perceptual information outside of one's focus of attention). This is in line with Hasher's access subfunction of inhibition; (b) cognitive inhibition (i.e., suppressing irrelevant information within working memory, such as suppressing unwanted thoughts). This is in line with Hasher's deletion sub-function of inhibition; (c) behavioural inhibition (i.e., withholding automatic responses). This is in line with Hasher's restraint sub-function of inhibition; and (d) oculomotor inhibition (i.e., withholding automatic eye movements). This is also in line with Hasher’s restraint sub-function of inhibition.

Wilson \& Kipp (1998). According to Wilson and Kipp (1998), inhibition can only function on information that is within the working memory storage system. "Inhibited items are first encoded into working memory, determined to be [relevant or] irrelevant and are then expelled from working memory” (p. 88-89). Since the items are within conscious awareness and subsequently removed, this is in line with Hasher and colleagues' deletion sub-function of inhibition. According to Wilson and Kipp (1998), however, if irrelevant information is kept out of working memory entirely, it is then 


\section{GENERAL INTRODUCTION}

referred to as 'resistance to interference.' Given this, Wilson and Kipp’s 'resistance to interference' is in line with Hasher's access sub-function of inhibition. Importantly, the definition of inhibition is not conceptually separate from interference. Recall that inhibition is defined as the mechanism by which interference is modulated (Dempster, 1992; Friedman \& Miyake, 2004). In this way, Wilson and Kipp’s 'resistance to interference' still falls within the definitional confines of inhibition.

Friedman \& Miyake (2004). The last framework of inhibition that will be discussed is proposed by Friedman and Miyake (2004), and consists of three subfunctions, namely prepotent response inhibition, resistance to distractor interference, as well as resistance to proactive interference. Prepotent response inhibition refers to stopping an automatic or dominant response from occurring, because it is not appropriate for the task at hand. This ability directly overlaps with the restraint sub-function proposed by Hasher and colleagues. Following in line with Wilson and Kipp (1998), Friedman and Miyake (2004) define resistance to distractor interference as the capacity to keep irrelevant information from disrupting task performance. This is said to be accomplished both by activating task-relevant stimuli, and, importantly, ignoring or suppressing task-irrelevant distracting stimuli. This concept loads nicely onto Hasher and colleagues' access sub-function of inhibition, but could also be interpreted as deletion, if the time at which the distracting information was suppressed has been specified as after it has entered working memory. Last, resistance to proactive interference involves the ability to manage intrusions from information that was task relevant at some earlier point in time, but has since become task-irrelevant. This type of inhibition maps nicely onto the deletion sub-function proposed by Hasher and colleagues. 


\section{GENERAL INTRODUCTION}

\section{Summary}

This section reviewed many different ways in which the cognitive construct inhibition has been segmented and defined in the literature. Importantly, all of the aforementioned models share two key features: (a) inhibition is not a unitary construct, it consists of a variety of different sub-functions; (b) there is substantial overlap and consistency across models, in terms of how the various sub-functions are defined. After examining various definitions of inhibition - across several different groups of researchers, it is apparent that all of the definitions can be mapped, fully or partially, onto the three sub-functions proposed in Hasher's model: access, deletion, and restraint. The first (i.e., access sub-function) blocks distracting, irrelevant information from conscious awareness or attention. The second (i.e., deletion sub-function) is the ability to identify and remove or suppress no longer relevant information from attentional focus. The third (i.e., restraint sub-function) is the capacity to withhold an automatic reflex or response. Given the overarching commonalities and the close connections across various models of inhibitory functioning in the literature, the current study will utilize the model of inhibition proposed by Hasher and colleagues (1999, 2007), which outlines three subfunctions of inhibition: access, deletion, and restraint, as the theoretical foundation.

\section{Age-related Deficits in Inhibition and the Associated Neural Correlates}

It has been well established in the literature that each sub-function of inhibition shows substantial age-related declines (for a review see Hasher et al., 1999; Hasher et al., 2007). The details of such findings will be elaborated on below, with special consideration given to the tasks used in the current dissertation. 


\section{GENERAL INTRODUCTION}

Access. The access sub-function of inhibition is commonly assessed in the literature using the Local-Global and Reading with Distraction tasks - both of which show age-related declines.

In the Local-Global task (e.g., Navon, 1977), participants are shown a large/global letter that is composed of small/local letters. They are instructed to attend to one dimension (i.e., local or global), while ignoring the other, and indicate whether the to-beattended dimension is one of two possible targets (e.g., 'A' or 'D'). In this task, there are three different types of trials: congruent, incongruent, and neutral. In congruent trials, the local and global dimensions are matched (e.g., a large 'A' made out of small 'A's). In incongruent trials, the local and global dimensions are conflicting (e.g., a large 'A' made out of small ' $\mathrm{D}$ 's) - recall the task instruction is to indicate the presence of an 'A' or a 'D'. Last, in the neutral trials, the local and global dimensions are not matched, but do not conflict with each other (e.g., a large 'A' made out of small 'H's; see Figure 1).

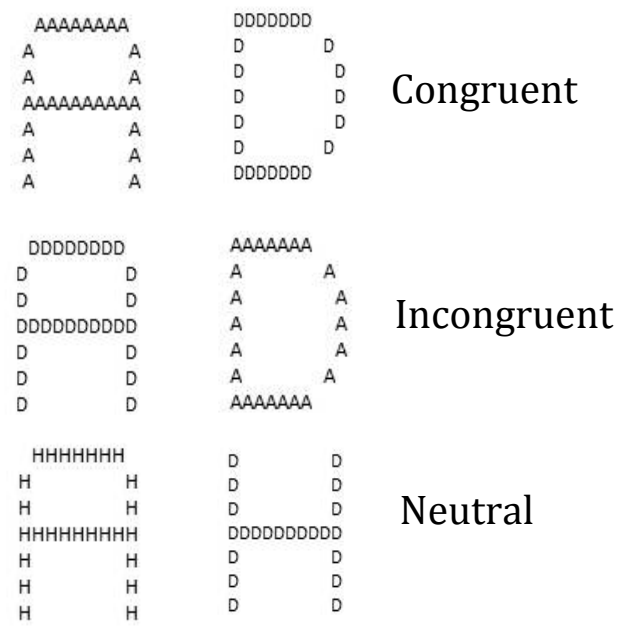

Figure 1. Sample of Local-Global stimuli for target letters 'A' or 'D', with global dimension focus on the left and local dimension focus on the right. 


\section{GENERAL INTRODUCTION}

Previous work with healthy young adults demonstrates faster responses to and more disruption from the global dimension as compared to the local dimension, which is referred to as the global precedence effect (Kotchoubey, Wascher, \& Verleger, 1997; Navon, 1977; Slavin et al., 2002; Thomas et al., 2007). Older adults, on the other hand, do not demonstrate such an effect (Thomas et al., 2007) or sometimes even show a local precedence effect (Slavin et al., 2002). Precedence effects in the Local-Global task can be measured in two ways: (a) faster overall reaction time (RT) to stimuli when attention is directed to the preferred dimension relative to non-preferred dimension, or (b) greater interference from the preferred dimension. A study by Thomas et al. (2007) demonstrated that young adults showed a global precedence effect with faster responses to and more disruption from global, as compared to local dimensions. In contrast, older adults showed an absence of global precedence - with no dimension differences. Slavin et al. (2002), however, showed a local precedence effect in older adults, with faster overall responses to and more distraction from local, as compared to from global dimension features. Following the seminal work of Navon (1977), the access sub-function of inhibition is indexed by the difference in RT between the incongruent and congruent trials (i.e., interference score) - with smaller differences representing better access abilities (also see Kotchoubey et al., 1997). Age-related deficits in access are predicted in the Local-Global task with greater overall interference in old, as compared to young adults (Thomas et al., 2007). Furthermore, older adults are expected to show larger deficits in access, when ignoring local as compared to global dimensions, while young adults are expected to show greater disruption from the global relative to local dimension of a stimulus. 


\section{GENERAL INTRODUCTION}

Another task that measures the access sub-function of inhibition is the Reading with Distraction task (e.g., Connelly et al., 1991). In this task, participants are asked to read a short passage out loud that contains both to-be-attended target information (in italicized letters) and to-be-ignored irrelevant information (not italicized). Task difficulty is modulated by the quality of the to-be-ignored information. Specifically, in the low distracting condition, the to-be-ignored information is strings of Xs and thus easy to keep out of one's focus of attention. Alternatively, in the high distracting condition, the distracting information is semantically related to the passage and thus is harder to ignore. For example, in a passage about a visit to the optometrist's office, the word 'secretary' is to-be-ignored. The access sub-function of inhibition is indexed by the difference in passage reading speed between the high and low distracting conditions, with smaller differences representing better access. Therefore, the ability to keep to-be-ignored information outside one's focus of attention limits the amount of disruption from the tobe-ignored passage appropriate words in the high distraction condition - thus reducing the passage reading speed difference score. Using this paradigm, Connelly et al. (1991) demonstrated that older adults' reading speed was more disrupted by the presence of high-distracting relative to low-distracting information, as compared to young adults. This finding supports age-related decline in the ability to keep irrelevant information from accessing attention.

Age differences in the access sub-function have also been found in neuroimaging studies. For example, a study by Gazzaley et al. (2005) clearly demonstrated age differences in access specific brain regions. Briefly, in this study, participants were shown a short sequence of stimuli: two faces and two natural scenes intermixed. Prior to 


\section{GENERAL INTRODUCTION}

the visual display, participants were given one of three instructions: (a) attend faces and ignore scenes; (b) attend scenes and ignore faces; and (c) passively view faces and scenes without trying to remember either. After a brief delay, participants were shown a probe display. In condition (a) and (b), they were asked to indicate whether or not the probe was one of the task relevant targets shown in the prior sequence. Condition (c) served as the control in which participants were shown a left or right pointing arrow and asked to indicate its direction. Overall, this task taps into the access sub-function of inhibition, because it assesses one's ability to keep task irrelevant, to-be-ignored stimuli (e.g., scenes, condition a) outside one's focus of attention. The neuroimaging data revealed less brain activation in the visual association cortex (VAC) of the parahippocampal/lingual gyrus when the stimulus with an 'ignore' instruction (e.g., scenes, condition a) was presented as compared to an 'attend' instruction (e.g., faces, condition a) in both young and older adults. Interestingly, it was only in the young adults that brain activation to the 'ignore' stimuli was found to be smaller relative to the 'passive viewing' (i.e., control condition c). Behaviourally, older adults showed both reduced accuracy and slowed RT on the working memory task - i.e., probe identification of scenes and faces. Taken together, these findings indicate that activity in the VAC of the left parahippocampal/lingual gyrus is equally activated during processing of task relevant information across age groups; however, suppression in this brain region, when successfully engaging the access sub-function of inhibition and keeping to-be-ignored information outside one's focus of attention, is impaired in older adults.

In a follow-up study by the same group of researchers (Gazzaley et al., 2007), functional connectivity analysis was used to evaluate the source of the dampening of 


\section{GENERAL INTRODUCTION}

brain activation in the VAC to 'ignore' stimuli. Top-down regulatory control signals originating from the prefrontal cortex (PFC) to the more posterior VAC brain regions were established. In other words, the PFC sends instructions to the medial temporal lobe, specifically the parahippocampal/lingual gyrus, to act as a gateway and open/encode relevant and close/dampen the signal from irrelevant information (Gazzaley et al., 2007). While the opening of the gate to task-relevant information seems not to show age-related decline, the suppression of signals in the VAC instructing the gate to close to task irrelevant ('ignore') information undergoes age-related changes (Gazzaley et al., 2005). These findings are in line with the neuroimaging research suggesting age-related degradation of specific brain regions, such as changes to the PFC (including the structure, white matter integrity and dopamine regulation mechanism), as well as hippocampal and parahippocampal regions of the brain (Bartzokis et al., 2003; Braver et al., 2001; Braver \& Cohen, 2000; Cabeza \& Dennis, 2013; DeCarli et al., 1994; Dempster, 1992; ErixonLindroth et al., 2005; Fjell et al., 2009; Head et al., 2004; Hedden, 2007; Hedden \& Gabrieli, 2004; Jack et al., 1998; Kemper, 1994; Pfefferbaum, Adalsteinsson, \& Sullivan, 2005; Raz, 2000; Raz, 2005; Raz et 1997; Resnick, Pham, Kraut, Zonderman, \& Davatzikos, 2003; Salat, Kaye, \& Janowksy, 1999, 2001; Salat et al., 2005; Suhara et al., 1991; Volkow et al. 1998; West, 1996). The specific brain mechanisms involved in 'closing the gate' to keep irrelevant information from accessing working memory (e.g., dopaminergic signals; D’Ardenne et al., 2012; see Badre, 2012 for a brief review), as well as how and why they change with age is beyond the scope of this dissertation. 


\section{GENERAL INTRODUCTION}

Deletion. The deletion sub-function of inhibition has been commonly measured with tasks including the N-Back and Directed Forgetting - both of which have shown age-related declines in performance.

Generally, in the N-Back task, participants are shown a series of individually presented stimuli (e.g., letters or numbers) and asked to indicate whether the current stimulus matches the one at $n$ trials back. This task involves the deletion sub-function of inhibition in that all of the stimuli enter working memory initially, but as the participant progresses through the sequence of stimuli, the no longer relevant items (e.g., items presented 4-Back in the sequence) should be removed from working memory. Task difficulty depends on the number of trials that must be kept in mind at a given time (typical range $=$ zero to three), while simultaneously removing earlier presented, but no longer relevant stimuli (Braver et al., 1997).

As an example, consider that a participant is shown the following series: 2-3-3-2 and asked to match trials presented 2-Back in the sequence. The first ' 2 ' in the example series is initially relevant and so must be kept in mind for several trials. However, by the time the last ' 2 ' in the series is presented, the first ' 2 ' is now 3-Back in the series, and thus should be deleted given the task instructions 'match 2-Back'. This last ' 2 ' in the sequence is considered a 3-Back foil, because it is a non-target item (i.e., not a 2-Back match), but it is identical to an item that was presented 3 items earlier in the sequence. If the first ' 2 ' in this series is not effectively deleted from working memory, the participant is more likely to incorrectly respond 'TARGET' to the second '2' (i.e., the 3-Back foil).

Seminal work by Kirchner (1958) investigated age differences in the N-Back task, including 0-Back to 3-Back conditions. However, many of the older adult participants 


\section{GENERAL INTRODUCTION}

that they tested had trouble completing the 3-Back condition. Kirchner found no age differences in the 0-Back condition, but incrementally greater age differences with greater N-back condition difficulty. Similarly, Dobbs and Rule (1989) found no age differences in accuracy for the 0-Back condition, but better performance in the young compared to the old for the 1-Back and 2-Back conditions. Verhaeghen and Basak (2005) compared performance of young and older adults using a modified N-Back task with conditions ranging from 1 to 5-Back. Overall accuracy performance was shown to decline with increasing $\mathrm{N}$ overall and, importantly, age differences expanded with increasing $\mathrm{N}$ across conditions.

Another task that assesses the deletion sub-function of inhibition is the Directed Forgetting task. In one form of this paradigm, participants are shown a list of words - one by one - followed by a cue, which instructs participants to either 'remember' (to-beremembered; TBR) or 'forget' (to-be-forgotten; TBF) the word for a later memory test. As is evident from this paradigm structure, participants first encode the word (i.e., it enters working memory) and then are subsequently informed to either remember it (i.e., store in memory for later use) or forget it (i.e., delete it from working memory). During the subsequent memory test, participants are surprisingly asked to recognize all of the words - including the ones that they were instructed to forget. The directed forgetting effect results in worse memory for TBF relative to TBR items, because the TBF words were effectively removed from working memory and thus cannot easily be retrieved during recognition.

Age-related declines in the deletion sub-function of inhibition have been demonstrated behaviourally using a Directed Forgetting task. Specifically, Zacks et al. 


\section{GENERAL INTRODUCTION}

(1996) demonstrated that older adults, as compared to young adults, showed better memory performance for TBF items during the later memory testing, suggesting that the TBF words are less effectively deleted from working memory by older than by young adults (Zacks et al., 1996).

Both of these deletion tasks (i.e., N-Back and Directed Forgetting) have been explored in the neuroimaging literature. In a meta-analysis conducted by Owen, McMillan, Laird and Bullmore (2005), based on 24 primary studies, brain regions activated during the N-Back task included the lateral and medial premotor cortex, dorsal cingulate, dorsolateral and ventrolateral prefrontal cortex, rostal prefrontal cortex or frontal poles, as well as the medial and lateral posterior parietal cortex. Not surprisingly, given the maintenance and manipulation of information in working memory required for the N-Back task, activation in frontal regions is dominant; however, parietal regions are also activated during this task. In addition, research by Mottaghy et al. (2000) used repetitive transcranial magnetic stimulation (rTMS) to mimic the effects of brain lesions, and administered a 2-back task, where participants were required to indicate whether the currently presented stimulus matched the one that was presented two trials earlier in the sequence. Mottaghy et al. (2000) demonstrated that stimulation to the dorsolateral prefrontal cortex (DLPFC) disrupted performance on the 2-Back working memory task, while rTMS to midline frontal did not. Additional analyses revealed that rTMS not only affected activity in the focal brain region (i.e., DLPFC); but, importantly, also disrupted activity in other regions, e.g., the parietal lobe. These rTMS findings support the down regulatory role of the PFC in executive control, such as selective attention, inhibition, and 


\section{GENERAL INTRODUCTION}

working memory tasks (also see Paus et al. 1997; see Miller \& Cohen, 2001 for a review).

Using a Directed Forgetting task, Wylie, Foxe and Taylor (2008) revealed that successfully forgotten TBF words were associated with increased activation in the right inferior frontal gyrus. This finding suggests that the deletion sub-function of inhibition is not simply an absence of encoding, but is rather an active cognitive process associated with greater activity in the right frontal gyrus brain region (also see Rizio \& Dennis, 2013). In line with these findings, Conway and Fthenaki (2003) demonstrated that patients with damage to the right frontal lobe were worse at intentionally forgetting information during a Directed Forgetting task. Findings from this lesion study, which link the deletion sub-function of inhibition to frontal brain regions, suggest that age-related deficits in the deletion sub-function of inhibition may, at least partially, be explained by age-related changes to the frontal regions of the brain (including the DLPFC) - e.g., a reduction in size and synaptic density, as well as changes to mechanisms that regulate dopamine (e.g., Braver \& Cohen, 2000; Cabeza \& Dennis, 2013; DeCarli et al., 1994; Dempster, 1992; Erixon-Lindroth et al., 2005; Hedden \& Gabrieli, 2004; Raz, 2000; Raz et al., 1997; Resnick et al., 2003; West, 1996).

Restraint. Last, the restraint sub-function of inhibition has been commonly measured with the Go-No Go and Stroop tasks. In the Go - No Go task, participants are shown a single stimulus on the screen (out of two possible stimuli) and are instructed to hit the space bar, when they see one stimulus ('Go' trial), and to withhold their response, when they see the other stimulus ('No Go' trial). In most paradigms, the 'Go' trials occur much more frequently than the 'No Go' trials, making the former more automatic and 


\section{GENERAL INTRODUCTION}

thus harder to withhold during the 'No Go' trials (Donders, 1969). The false alarm rate (i.e., hitting the space bar on a 'No Go' trial) is used to index the restraint sub-function of inhibition, with a smaller false alarm rate indicating better performance.

Surprisingly, published research on age differences in the Go-No Go task - as indexed by the false alarm rate - is sparse. Of those few studies that do report such data, no age differences have been reported (e.g., Allen, Weber, \& Madden, 1994; Falkenstein, Hoormann, \& Hohnsbein, 2002). The lack of age effects, however, may be explained by the equivalent probabilities for 'Go' and 'No Go' trials included in these paradigms.

Nevertheless, there is some empirical support for age differences in Go-No Go. For example, research using a modified Go-No Go task, requiring responses only to alternating target letters, trending age differences were demonstrated in the restraint subfunction of inhibition (worse performance with increasing age) and additional recruitment of frontal brain regions in older adults (not evident in young adults; Nielson, Langenecker, \& Garavan, 2002). This finding offers some support for the age-related decline in the restraint sub-function of inhibition as measured by the Go-No Go task. Additional support for age-related declines in the restraint sub-function of inhibition can be found with a task similar to the Go-No Go task, called the sustained-attention-toresponse task (SART; Robertson, Manly, Andrade, Baddeley, \& Yiend, 1997). In this task, participants are presented with a single digit - ranging from 1 to 9, and instructed to press a response button to every digit, except to an infrequent 'No-Go' stimulus (e.g., 3). A study conducted by McAvinue et al. (2012) examined performance in this task across the lifespan. Of interest herein is the relation between age and the number of times participants pressed the response key on the 'No-Go' trials (i.e., false alarms). Using 


\section{GENERAL INTRODUCTION}

pseudo-random trial presentation, it was demonstrated that the false alarm rate followed a ' $U$ ' shape pattern across the lifespan, with high rates in childhood and late adulthood. They also compared false alarm rates in young (i.e., in their 20s and/or 30s) to older adults (i.e., in their 60s and/or 70s) and found significantly higher levels in the latter (McAvinue et al., 2012). Although this study does not use the typical Go-No Go task paradigm design, it does support a reduction in the capacity to withhold inappropriate motor responses in older adults.

Another task used to measure restraint is the Stroop task. In this task, participants are instructed to make their responses based on the ink colour of the stimuli. There are three types of stimuli in a typical Stroop task: congruent (e.g., 'GREEN' in green ink), incongruent (e.g., 'GREEN' in blue ink), and neutral (e.g., 'XXXXX' in green ink). Since the word and colour information is contradictory on incongruent trials, participants must suppress the automatic word reading responses in order to correctly identify the ink colour of the word. Inhibitory efficiency is measured by a Stroop interference score, which quantifies the difference in performance (RT or accuracy) between incongruent and neutral trials. This score reflects one's ability to withhold the automatic reading response - with smaller interference reflecting better restraint (Hasher et al., 2007; Jonides et al., 2002; MacLeod \& MacDonald, 2000). Literature consistently shows higher Stroop interference scores for older as compared to young adults (e.g., Davidson, Zacks, \& Williams, 2003; Dulaney \& Rogers, 1994; Milham et al., 2002; West \& Alain, 2000;

for a review see MacLeod, 1991), indicating poorer restraint capabilities with increased age. 


\section{GENERAL INTRODUCTION}

Neuroimaging data has been evaluated in both the Go-No Go, as well as the Stroop task. Rubia et al. (2001) demonstrated the role of the DLPFC and the anterior cingulate cortex (ACC; also located in the frontal brain region) during a Go-No Go task. In addition, they established involvement of the pre-supplementary motor area (preSMA), inferior frontal, and inferior parietal cortices. The involvement of the frontal cortex during the restraint sub-function of inhibition is not disputed in the literature (e.g., Casey et al., 1997; for a review see Grafman, 2006); however, some researchers have suggested a predominant role of the right inferior frontal cortex (e.g., Chikazoe et al., 2009; for a review see Aron, Robbins, \& Poldrack, 2004).

For the Stroop task, neural patterns during incongruent trials revealed activation in the ACC, insula, premotor and inferior frontal regions (Leung, Skudlarski, Gatenby, Peterson, \& Gore, 2000). Interestingly, Milham et al. (2002) investigated age-related changes in brain activation patterns during the Stroop task. They found reduced involvement of the DLPFC during conflict processing (i.e., incongruent trials where the colour word and ink colour are mismatched) in older adults as compared to young adults. Furthermore, activity in the ACC was shown to be specifically sensitive to conflict elicited during the presentation of incongruent trials in young adults, while in older adults ACC activity was elicited during both incongruent and congruent trials. Reduced sensitivity in response to conflicting stimuli may explain age-related performance decrements in the restraint sub-function as measured with the Stroop task.

As demonstrated above, higher-order cognitive processes, including the three subfunctions of inhibition (access, deletion, and restraint), involve the frontal lobes of the brain (e.g., PFC, ACC), which undergo a host of age-related changes including reduced 


\section{GENERAL INTRODUCTION}

size, degradation of white matter integrity and decreased availability of dopamine (Bartzokis et al., 2003; Braver et al., 2001; Cabeza \& Dennis, 2013; Dempster, 1992;

DeCarli et al., 1994; Erixon-Lindroth et al., 2005; Head et al., 2004; Hedden, 2007; Hedden \& Gabrieli, 2004; Kemper, 1994; Pfefferbaum et al., 2005; Raz, 2000; Raz, 2005; Raz et 1997; Resnick et al., 2003; Salat et al., 1999, 2001; Salat et al., 2005; Suhara et al., 1991; Volkow et al. 1998; West, 1996). The connection between brain changes and behavioural performance may explain the vulnerability of older adults to deficits in inhibition and further underscores a need to target this cognitive ability for intervention. Furthermore, exploring the plasticity of inhibition across all three sub-functions in older adults is important, because each works to control the contents of working memory at a different point along the information processing stream; therefore, all of these components may need to be trained as a whole in order for true benefits to be observed. In addition, the link between inhibition and performance in other cognitive domains known to decline with age, such as processing speed and memory, further highlights the need to evaluate training gains in inhibition among older adults. This connection has been elaborated on in the inhibitory deficit hypothesis of aging, as initially proposed by Hasher and Zacks (1988; also see Hasher et al., 1999; Hasher et al., 2007), and will be further described in the section below.

Inhibitory deficit hypothesis of aging. Age-related declines in inhibitory subfunctions are consistently demonstrated and have been widely implicated in the aging literature as underlying deficits in other cognitive domains (e.g., Dempster, 1992; Gazzaley, et al., 2005; Hasher et al., 1999; Hasher et al., 2007). One prominent theory supporting this association is the inhibitory deficit hypothesis of aging. According to this 


\section{GENERAL INTRODUCTION}

theory, deficits in the ability to keep the working memory storage system clear of irrelevant information is fundamental for efficient cognitive functioning in other domains, including processing speed and memory (Hasher \& Zacks, 1988; Hasher et al., 1999; Hasher et al., 2007; also see Dennis, 2006). Other researchers, including Wilson and Kipp (1998), have also referred to this association, but termed it the inefficient inhibition hypothesis, and discuss it in reference to young children (also see Bjorklund \& Kipp Harnishfeger, 1990; Kipp Harnishfeger, 1995; Kipp Harnishfeger \& Bjorklund, 1993).

The inhibitory deficit hypothesis is best understood in the context of a limited resources model (Case, 1985; Case, Kurland, \& Goldberg, 1982; Craik, 1986; Craik \& Byrd, 1982; Dennis, 2006). This model suggests that we have limited cognitive resources available to both store and process information (Hasher \& Zacks, 1979; Schneider \& Schiffrin, 1977; Kipp Harnishfeger, 1995; Wilson \& Kipp, 1998). Furthermore, the amount of resources available for cognitive operations is thought to decrease with age; therefore, the reduced attentional resources model would predict age-related declines in cognitively demanding tasks such memory (e.g., working memory storage and processing, episodic memory encoding and retrieval; Craik, 1983; Craik \& Byrd, 1982; Craik \& Jennings, 1992). According to the inhibitory deficit hypothesis of aging, however, it is not the size of the pool of resources available for cognitive activity that changes with age, but the efficiency with which the available resources are used (Hasher \& Zacks, 1988; Hasher et al. 1999; Hasher et al., 2007; Kipp Harnishfeger, 1995; Wilson \& Kipp, 1998). 


\section{GENERAL INTRODUCTION}

In accordance with this line of thinking, a study by May, Hasher, and Kane (1999) demonstrated that working memory performance (i.e., storage and manipulation of information), as measured by various span tasks, could be altered by modifying the experimental paradigm. Although past research shows that resource demanding working memory abilities decline with age (e.g., Gick, Craik, \& Morris, 1988; Salthouse \& Babcock, 1991), May et al. (1999) eliminated age differences in working memory performance by reducing the influence of no longer relevant information. In particular, because older adults show deficits in the deletion sub-function of inhibition, no longer relevant information is not efficiently removed from their working memory. Therefore, information that was previously relevant - during earlier span task trials - filled up the working memory storage space, resulting in an apparent age-related reduction in working memory capacity. However, by flipping the order of trial presentation to descending span size (i.e., large to small) - as opposed to the typical ascending presentation (i.e., small to large), the working memory clutter that gathered across trials - and assumedly made large span size trials especially challenging - was experimentally reduced. This resulted in improved performance in older adults and demonstrates the role of inhibition in modulating storage and processing capacities within working memory (May et al., 1999).

Furthermore, inhibitory deficits have also been shown to affect measures of processing speed. Lustig, Hasher, and Tonev (2006) modified a speed of processing measure, the Digit Symbol, to create two computerized versions of the task - one high distracting and one low. The high distracting condition was similar to the original paperpencil version of this task in that participants were simultaneously presented with digits in squares, each with a corresponding empty square underneath (4 rows of 25 items, 


\section{GENERAL INTRODUCTION}

including seven practice trials). In the low distracting condition, items were presented one at a time on the computer screen. In both conditions, participants were required to identify the corresponding symbol based on the number conversion code presented at the top of the screen. Results demonstrated that young adults performed at the same speed in both conditions, while older adults' speed was significantly improved in the low distracting condition. The findings are interpreted in the context of the inhibitory deficit hypothesis of aging, whereby reduced ability to keep to-be-ignored information outside one’s focus of attention (access) negatively impacts processing speed in older adults.

Additional evidence of the association between inhibitory efficiency and cognitive performance can be found in the study by Gazzaley et al. (2005) - discussed above. To briefly reiterate, using fMRI it was demonstrated that older adults were not effectively suppressing task-irrelevant information, and also showed poorer working memory performance to task relevant information (i.e., slower and less accurate). This was in comparison to young adults who demonstrated active suppression of task irrelevant information and better working memory performance to task relevant information. Taken together, these findings demonstrate a link between effectiveness of the access sub-function of inhibition and efficiency of working memory performance.

Following these lines of research, a core assumption of the inhibitory deficit hypothesis is that age-related deficits in inhibition impact the storage and processing capacity of working memory, which leads to declines in a variety of other cognitive domains. As a result of older adults' inhibitory deficits, valuable cognitive resources are being used to store and process task irrelevant information, at the cost of storing and processing task relevant information. The inefficient use of limited resources in older 


\section{GENERAL INTRODUCTION}

adults thereby restricts speed and accuracy of processing and impacts a variety of cognitive domains, including memory (Bjorklund \& Kipp Harnishfeger, 1990; Dempster, 1992; Gazzaley et al., 2005; Hasher \& Zacks, 1988; Hasher et al., 1999; Hasher et al., 2007; Lustig et al., 2006; May et al., 1999; Wilson \& Kipp, 1998). Given the central importance of inhibition in cognitive functions and the prevalent age-related declines in the various sub-functions of inhibition, the goals of this dissertation are threefold: (a) to evaluate the durability of single sub-function of inhibition training in older adults, (b) to examine the retest practice effect of training three sub-functions of inhibition in older adults, and (c) to examine whether training of inhibition can enhance other cognitive domains. As discussed above, the three sub-functions of inhibition work to control the contents of working memory at different points in time along the information processing stream; therefore, it is expected that training all three (access, deletion, and restraint) concurrently will highly encourage transfer to other cognitive domains, such as memory and processing speed. Furthermore, the current research serves to add to the empirical literature on the association between inhibition and other cognitive domains through an examination of transfer effects following inhibition training.

\section{Cognitive Plasticity of the Aging Brain}

Research demonstrates that older adults maintain the capacity to slow down or even reverse age-related cognitive declines with training (e.g., Baltes \& Lindenberger, 1988; Thompson \& Foth, 2005). Cognitive maintenance in older age is important for several reasons, including keeping an independent life and living in the community. Furthermore, declines in cognitive abilities, including executive functions, have been shown to precede a diagnosis of probable or possible Alzheimer's disease by several 


\section{GENERAL INTRODUCTION}

years (Bäckman, Jones, Berger, Jonsson Luakka, \& Small, 2005). With 106.2 million people projected to have probable or possible Alzheimer’s disease by 2050 (Brookmeyer, Johnson, Ziegler-Graham, \& Arrighi, 2007), the pursuit of effective methods for maintaining or even enhancing cognitive functioning in older adults is of significant importance. One such method is cognitive training (e.g., practice on tasks requiring a specific cognitive ability). In older adults, cognitive training has been explored and shown to be successful in a variety of domains including reasoning, processing speed, visual attention, and memory (e.g., Ball et al., 2002; Baltes, Sowarka, \& Kliegl, 1989; Edwards et al., 2005; Mahncke et al., 2006; Yang, Krampe \& Baltes, 2006; Yang, Reed, Russo \& Wilkinson, 2009a).

Plasticity of executive functions. Of particular importance to the current dissertation, cognitive training with older adults has also been shown to be effective in executive functions, such as dual-task processing (e.g., Bherer et al., 2005), updating (e.g., Dahlin, Nyberg, Bäckman, \& Stigsdotter Neely, 2008a), and task-switching (e.g., Kramer, Hahn, \& Gopher, 1999) - each of which will be described briefly below.

Bherer et al. (2005) examined training related gains across five 1-hour sessions (frequency not specified) of dual-task processing in young and older adults. In general, dual-task performance is assessed by one's ability to complete two tasks concurrently. In Bherer et al. (2005), participants were asked to classify a letter (i.e., as 'C' or 'B'), while simultaneously deciding whether a tone was high or low. Amazingly, the authors found similar training gains between young and older adults in terms of RT performance and even greater gains in older adults in terms of accuracy. This study supports the plasticity of dual-task processing in older adults. 


\section{GENERAL INTRODUCTION}

Dahlin et al. (2008a) explored the plasticity of updating - i.e., the ability to refresh the contents of working memory - in young and older adults. In this study, participants completed fifteen 45-minute training sessions (three sessions per week, for five weeks) in which they were presented with sequences of stimuli (e.g., letters, numbers) of varying length. They were instructed to continuously update their working memory with the last four presented items. At an unknown time, the list ended and participants were required to recall the last four items from the list as quickly as possible. Performance was shown to improve in both age groups following the training - with greater improvements in the young as compared to the older adults. Regardless, these findings offer evidence for the plasticity of updating in older adults.

Kramer et al. (1999) explore the plasticity of task switching in young and older adults across four training sessions (within one week; session duration not specified). In this study, participants were presented with number strings of varying length (e.g., '3333’ or '33'). Participants were instructed to either respond in accordance the value of the digit (i.e., is the digit greater than or less than ' 5 ') or in accordance to the number of digits presented (i.e., are there more or less than five digits presented). The dependent variable of interest was the switching costs, indexed by the RT difference between switch trials (when the task instructions changed from one trial to the next) and non-switch trials (when the task instructions stayed the same from one trial to the next). Older adults demonstrated significant reductions in switching costs across training sessions, supporting the existence of plasticity.

Plasticity of inhibition. Despite the well-documented age-related deficits in the various types of inhibition, and their close connection to other cognitive functions, as 


\section{GENERAL INTRODUCTION}

well as their distinct roles within the information-processing stream of working memory, it is surprising that, to-date, no known research has systematically explored the plasticity of all three sub-functions of inhibition in an aging population. This, therefore, is the main focus of my dissertation.

Earlier studies have, however, explored the plasticity of inhibition using a singletask approach (e.g., Davidson et al., 2003; Dulaney \& Rogers, 1994; Li et al., 2008). Li et al. (2008) trained young and older adults using a spatial N-Back task across 45 daily practice sessions (15 minutes/day). Both young and older adults showed substantial improvements in accuracy performance as a result of the training. Furthermore, others including Davidson et al. (2003), as well as Dulaney and Rogers (1994) - have used the Stroop task to train inhibition in older adults and demonstrated improvements within a single session or across two sessions. Extending the Stroop training literature, my Master's thesis (Wilkinson \& Yang, 2012) sought to explore the plasticity of inhibition across six retest sessions - controlling for item-specific effects by using varying colours at different sessions. The results revealed that older adults were able to improve inhibitory processing, as indexed by reduced Stroop interference scores, with practice; however, little is known about whether the training benefits of single-task inhibition training could be maintained beyond the original training sessions. The first chapter of this dissertation will fill this gap.

Long-term maintenance of training gains. It has been demonstrated that older adults are able to maintain cognitive training effects for long periods of time ranging from several months (e.g., Borella, Carretti, Riboldi, \& De Beni, 2010; Brehmer, Westerberg, \& Bäckman, 2012; Dahlin et al., 2008a; Günther, Schäfer, Holzner, \& 


\section{GENERAL INTRODUCTION}

Kemmler, 2003; Kramer et al., 1999; Li et al., 2008; Yang \& Krampe, 2009) up to several years (Ball et al., 2002; Stigdotter Neely \& Bäckman, 1993; Willis et al., 2006; Willis \& Nesselroade, 1990). Although some of these studies established maintenance effects following the inclusion of booster sessions - i.e., additional training sessions months or years after the initial training (e.g., Ball et al., 2002; Willis et al., 2006; Willis \& Nesselroade, 1990; Yang \& Krampe, 2009), others have demonstrated durability without the use of booster sessions (e.g., Borella et al., 2010; Brehmer et al., 2012; Dahlin et al, 2008a; Li et al., 2008; Stigdotter Neely \& Bäckman, 1993). For example, older adults demonstrated maintenance of memory training gains, above baseline levels, for three years without any booster training (Stigdotter Neely \& Bäckman, 1993).

The durability of training effects in older adults has been demonstrated in a variety of cognitive domains, including memory, reasoning, perceptual-motor speed and visual attention (Günther et al., 2003; Willis \& Nesselroade, 1990; Stigdotter Neely \& Bäckman, 1993; Willis et al., 2006; Yang \& Krampe, 2009). It has also been demonstrated in executive functions, such as updating or the deletion sub-function of inhibition (Dahlin et al., 2008a; Li et al., 2008); however, to our knowledge, long-term maintenance of training gains in restraint has not yet been explored in older adults. The first chapter of this dissertation will serve to fill this gap by following up on the Wilkinson and Yang (2012) study to examine the durability of Stroop training across one and three years.

Transfer effects. Broadly speaking, transfer is the generalizability of the skills learned during the training to other contexts (Baltes, Sowarka \& Kliegl, 1989; Willis, Blieszner, \& Baltes, 1981). Wilkinson and Yang (2012) evaluated the transfer effects of 


\section{GENERAL INTRODUCTION}

inhibition training to other untrained cognitive abilities, including inhibition (using Go No Go), speed of processing (Digit Symbol), reasoning (Letter Series), attention (Attention Network Test), and task-switching (Task-Switching task). The analyses revealed that inhibition training using the Stroop task did not transfer to other untrained cognitive domains. Theoretically speaking, in the context of the inhibitory deficit hypothesis of aging (Hasher et al., 1999; Hasher et al., 2007), improvements in inhibition should have freed-up cognitive resources by limiting the presence of task-irrelevant information. This, therefore, should have resulted in performance improvements in other cognitive domains, such as to processing speed. One potential explanation for the lack of transfer following Stroop training in Wilkinson and Yang (2012) is the lack of overlap between the training and the transfer tasks.

Previous work indicates that transfer is most likely to occur when the training and transfer tasks share common underlying processes (e.g., Edwards et al., 2005; Jaeggi, Buschkuehl, Jonides, \& Perrig, 2008). For example, Dahlin, Stigsdotter-Neely, Larsson, Bäckman, and Nyberg (2008b) suggest that successful transfer requires an overlap in cognitive processes as well as associated brain regions. Therefore, one potential explanation for the lack of transfer found in older adults following inhibition training using the Stroop task in Wilkinson and Yang (2012) is that the activated brain network following the training may have been too narrow, because only the restraint sub-function of inhibition was trained, and the cognitive processes enlisted during the Stroop task did not sufficiently overlap with those assessed in the transfer tasks. Given this, the lack of elicited transfer may have been the result of the limited number of inhibitory subfunctions that were trained - i.e., not including access and deletion, which limited the 


\section{GENERAL INTRODUCTION}

extent of brain activation within the PFC and across associated brain networks, ultimately restricting transfer effects. Furthermore, the three sub-function of inhibition are said to control the contents of working memory at different stages along the information processing stream (Hasher et al., 1999; Hasher et al., 2001; Hasher et al., 2007); therefore, it is also possible that all three types of inhibition need to be trained concurrently to improve processing across all stages and encourage transfer to other cognitive domains. In addition, the Stroop task is not process pure given its cognitive complexity (i.e., response inhibition, interference resolution, and goal maintenance; Buitenweg, Murre, \& Ridderinkhof, 2012). Therefore, the segment of the Stroop task that does involve purely inhibitory abilities may not have overlapped enough to promote transfer to other untrained tasks. Following these lines of thinking, the Stroop task may have failed to activate an inhibitory processing brain network that was wide enough to elicit improvement in the untrained mental tasks that are theoretically associated with efficient inhibition.

To follow up on this possibility and to broaden the potential transfer effects, the current dissertation will evaluate the plasticity of all three sub-functions of inhibition (access, deletion, and restraint), which will encompass a broader set of mental functions and involve a wider associated brain network for the evaluation of potential transfer effects. To my knowledge, no previous studies have used a multiple tasks approach to train all three sub-functions of inhibition; therefore, this dissertation takes a unique and novel approach to improving inhibition among older adults.

Specifically, a hierarchical pattern of transfer effects: near-near, near, and far transfer will be explored in this dissertation. This continuum of transfer is based on the 


\section{GENERAL INTRODUCTION}

structural similarity and cognitive overlap between the transfer and the training tasks (Willis et al., 1981). Using this approach, I will be able to tease apart which component (structure, cognitive abilities, or both) is most critical to elicit transfer in older adults. In general, near-near transfer involves training and transfer tasks that overlap in both structure and cognitive processes; near transfer involves training and transfer tasks that overlap only in the required cognitive ability, but not in task structure; and finally, far transfer involves training and transfer tasks that do not overlap in either cognitive ability or task structure - although distant cognitive associations between the training and transfer tasks are typically drawn.

In the literature, it is well established that the nearer the transfer task (i.e., the greater the overlap between the training and transfer tasks in both task structure and cognitive abilities), the greater the likelihood of eliciting transfer effects. To illustrate this point, let's look at examples from each type of transfer along the continuum. First, nearnear transfer refers to the training-induced performance improvement on a transfer task outcome that is the same (in task structure and cognitive operation) as the trained task (often, however, with varying items). This type of transfer has successfully been demonstrated in the training literature in older adults (Baltes et al., 1989; Willis et al., 1981), and, more recently, has been elicited in this population following executive function training such as dual-task processing (e.g., Bherer et al., 2005), task-switching (Karbach \& Kray, 2009), and spatial N-back training (Li et al., 2008). Near-near transfer is the easiest to elicit because transfer is optimized when the cognitive operation being trained maximally overlaps with that being utilized in the transfer tasks (Thorndike \& Woodworth, 1901). 


\section{GENERAL INTRODUCTION}

Near transfer, on the other hand, refers to pretest - posttest performance improvements on transfer tasks that are structurally different, but theoretically measure the same cognitive abilities as the ones implemented during the training. Near transfer is harder to elicit than near-near transfer and is most typically shown only in young adults. For example, training on a letter memory updating task showed near transfer to the NBack task in young, but not older adults (Dahlin et al., 2008a; Dahlin et al., 2008b). However, Li et al. (2008) demonstrated near transfer in older adults to a numerical Nback task following spatial N-back training. The discrepancy in near transfer effects found in older adults may have to do with the quantity and frequency of the training regime. For example, Dahlin et al. (2008a) trained participants across 15 sessions (3/week for five weeks), while in Li et al. (2008), participants were trained daily for 45 days. Therefore, it is possible that the increased frequency of the training program used in Li et al. (2008) promoted greater transfer through a more constant training routine. Dahlin et al. (2008b), however, suggest that age-related limits in near transfer may be due to the changes in associated brain structures involved in the training and transfer tasks.

Last, far transfer refers to pretest - posttest improvement in transfer tasks that are structurally different from, and tap different cognitive abilities than those being trained (Karbach \& Kray, 2009). This type of transfer is rarely elicited in older adults (e.g., Dahlin et al., 2008a; Li et al., 2008), but it is possible. One such example is Karbach and Kray (2009) who found far transfer from executive function task-switching training to other tasks measuring inhibition, spatial working memory and reasoning. The authors theorized that the far transfer effects that they found (even in older adults) were due to the amount of executive processes that were trained using a task-switching paradigm (e.g., 


\section{GENERAL INTRODUCTION}

goal maintenance, task-set selection and ignoring irrelevant information), which were cognitively associated with the far transfer outcome measures.

In sum, the above findings suggest that transfer effects are most often established when the transfer tasks are identical to those used during the training (i.e., near-near transfer). The more distinction (structural and/or cognitive) between the training and transfer tasks (i.e., near and far transfer), the smaller the likelihood of eliciting transfer effects - especially in older adults. To my knowledge, no studies to date have compared the different gradients of transfer effects (i.e., near-near, near and far) in older adults following inhibition training. The current dissertation will fill this gap by training older adults across three sub-functions: access (using the Local-Global), deletion (N-Back), and restraint (Go-No Go) and then assessing near-near transfer using Local-Global, N-Back and Go-No Go with varying items, near transfer using Reading with Distraction, Directed Forgetting and Stroop, and far transfer using Corsi Block (working memory), Word List Recall (episodic memory), Letter Series (reasoning) and Digit Symbol (processing speed).

\section{Dissertation Structure}

This dissertation consists of three chapters. Chapter 1 presents a study that examines the long-term maintenance of retest learning of inhibition in older adults over one and three year periods. It serves to examine the durability of single sub-function of inhibition training (using the Stroop task) in older adults and is a follow-up study to Wilkinson and Yang (2012). Chapter 2 examines the age differences in the three subfunctions of inhibition. All participants within each age group completed the same series of cognitive measures - known in the literature to tap the three sub-functions: access, 


\section{GENERAL INTRODUCTION}

deletion, and restraint. Finally, Chapter 3 presents a study that examines the plasticity of all three sub-functions of inhibition - in most of the older adults tested in Chapter 2 through retest practice and the associated transfer effects. The Ryerson Research Ethics Board (REB) approved all of the studies included in this dissertation (see Appendices A, $\mathrm{B}$, and $\mathrm{C}$ for the REB approval notices for Chapters 1, 2, and 3, respectively). The following exclusion criteria were implemented across the three studies: age (i.e., less than 17 or over 30 years of age for young adults and younger than 60 years of age for older adults); language (i.e., English learned after the age of 6); neurological disorders (e.g., stroke, dementia); a current diagnosis of a psychological disorder (e.g., depression or anxiety); taking medication(s) that impact cognition (e.g., Prozac, Ritalin); unconscious for more than five minutes; uncontrolled medical condition(s) (e.g., diabetes, cardiovascular disease); colourblindness; a Shipley vocabulary score lower than 20; and/or participation in any cognitive training programs within the last five years.

Each chapter is presented as a separate study, including four sections: a brief Introduction, Method, Results, and Discussion. In addition, a General Conclusions section is included at the end of this dissertation to briefly summarize the results, as well as outline implications of the research findings. Recommendations for future research in cognitive training geared towards older adults are also discussed. 


\section{LONG-TERM MAINTENANCE}

Chapter 1. Long-term Maintenance of Retest Learning of Inhibition in Older Adults:

1-year and 3-year Follow-up

This study examined the long-term maintenance of retest learning benefits in inhibition, as measured with the Stroop task, in older adults over one and three year periods. Long-term maintenance of cognitive training effects is important because it demonstrates endurance of the learning induced by the initial training. As reviewed in the General Introduction, empirical studies have shown endurance of training gains in older adults across a variety of time periods, ranging from several months (e.g., Borella et al., 2010; Brehmer et al., 2012; Dahlin et al., 2008a; Günther et al., 2003; Kramer et al., 1999; Li et al., 2008; Yang \& Krampe, 2009) all the way up to several years (Ball et al., 2002; Stigdotter Neely \& Bäckman, 1993; Willis et al., 2006; Willis \& Nesselroade, 1990), and even in the absence of booster sessions (e.g., Borella et al., 2010; Brehmer et al., 2012; Dahlin et al, 2008a; Li et al., 2008; Stigdotter Neely \& Bäckman, 1993).

This study is a follow-up to Wilkinson and Yang (2012), where 42 healthy older adults were trained on inhibition using the Stroop task. Specifically, in the original study, participants were assigned to one of three feedback groups: summary feedback, individualized and adaptive feedback, or a no-feedback control. The whole experiment was spread across 4 weeks. All participants first completed a 1.5-hour pretest session (in the week right before training), followed by six 30-minute retest practice sessions (three per week across two weeks) on the Stroop task, and last completed a 1-hour posttest session (in the week immediately following the training). During the pretest and posttest sessions, participants completed a battery of cognitive tasks, as well as some demographic and background questionnaires. 


\section{LONG-TERM MAINTENANCE}

During the retest practice sessions, the feedback manipulation was implemented during the 'training' block (see Materials and Procedure section below for more details). For those in the individualized and adaptive feedback group, following every correct response, participants received feedback on accuracy, RT, as well as a bar graph which depicted whether performance on the current trial was better (green bar) or worse (red bar) than the average of all previous trials. The height of the bar represented the size of this difference. In addition, at the end of this block, participants in both the individualized and adaptive as well as the summary feedback groups were reminded to always try to improve their speed and accuracy at every session, and were also informed of their average speed and percentage of correct responses for the block. An incorrect response was followed by accuracy feedback only. Those in the no-feedback control group did not receive any feedback during or at the end of this block. In addition, the Stroop colours varied across the six retest practice sessions to minimize item-specific effects (for additional details see Wilkinson \& Yang, 2012).

The original training results demonstrated improved inhibition across the six sessions - as indexed by reduced Stroop interference scores (i.e., the difference in performance between incongruent and neutral trials). Interestingly, this effect was found to be equivalent across all three feedback groups, suggesting that the type and amount of externally generated feedback did not modulate training related improvements in inhibition. As a follow-up, the current study aimed to examine the long-term maintenance of retest learning in inhibition among older adults one and three years after the original training. The maintenance of training will be assessed in terms of performance at the two follow-up sessions - one year and three year delay after the original training - in 


\section{LONG-TERM MAINTENANCE}

comparison with the original training gains, as well as baseline performance. In light of previous findings (e.g., Dahlin et al., 2008a; Stigdotter Neely \& Bäckman, 1993), it is expected that the original training gains will be maintained at both the 1-year and 3-year follow-up sessions - where performance levels are expected to continue to exceed baseline performance levels.

\section{Method}

\section{Participants}

The original training sample consisted of 42 older adults (28 females, age range $=$ $60-84$ years; mean $[M]=70.98$, standard deviation $[S D]=6.42$ ) that were recruited from the internal Ryerson Senior Participant Pool. All participants provided informed consent according to the REB at Ryerson University (see Appendices D and E for the consent agreements for the 1-year and 3-year follow-up sessions, respectively). Participants were evenly divided into three training groups (i.e., summary feedback, individuals and adaptive feedback, and no-feedback). Given the lack of feedback effects in the original training study, as well as the small sample size per feedback group within each follow-up sample, the data presented herein were collapsed across feedback groups.

The 1-year follow-up sample. Thirty-three of the 42 participants from Wilkinson and Yang (2012) returned for the 1-year follow-up session (21 females, age range = $60-84$ years, $M=71.12, S D=6.52$ ). All demographic information and baseline cognitive performance is displayed in Table 1. There were no attrition effects, because the participants who returned for the 1-year follow-up session $(n=33)$ and those who opted not to return $(n=9)$ did not differ in their performance on any baseline cognitive measures or demographic variables collected at pretest (see Table 1). For example, the 


\section{LONG-TERM MAINTENANCE}

ratio of males to females was the same in the sample that chose to come back for the follow-up and those that did not.

The 3-year follow-up sample. One participant was accidentally tested two years after the original training; so, their data were removed from the analyses. Twenty-six of the 33 participants who were tested at the 1-year follow-up returned for the 3-year follow-up session (16 females, age range $=60-80$ years, $M=69.81, S D=5.91$ ). At the 3-year follow-up session, one participant was suspected for possible colour blindness, as indicated by difficulty in answering more than three items on the Dvorine PseudoIsochormatic Plates (1953). This individual, however, showed similar accuracy scores (> 87\%) on the Stroop task as compared to all other participants at the 3-year follow-up; therefore, their data were not excluded from the final analysis. Again, there were minimal attrition effects, because the participants that returned for the 3-year follow-up session ( $n=26)$ and those who opted not to return $(n=15)$ did not differ in their performance on most baseline cognitive measures or demographic variable data collected (e.g., gender ratio) at pretest (see Table 1). However, the 3-year follow-up sample scored significantly higher (suggesting better performance) on the orienting component of the Attention Network Test (ANT; Fan, McCandliss, Sommer, Raz, \& Posner, 2002) relative to those who did not return for the 3-year follow-up session. Correlations were run between the ANT orienting and Stroop interference scores from all of the training session, as well as the two follow-up sessions for those in the 3-year follow-up sample. None of the correlations were significant (all $p s>.20$ ), suggesting that the ANT orienting scores were not likely contributing to the Stoop interference scores. This baseline cognitive variable was thus not considered further in the final data analyses. 


\section{LONG-TERM MAINTENANCE}

All participants were compensated $\$ 10$ for each hour of participation and debriefed (orally) at the end of each session (see Appendix F). 


\section{LONG-TERM MAINTENANCE}

Table 1

Follow-up vs. No Follow-up Participant Demographic Characteristics and Baseline Cognitive Performance Assessed at Pretest

\begin{tabular}{|c|c|c|c|c|c|c|c|c|}
\hline \multirow[b]{2}{*}{ Characteristic } & \multicolumn{4}{|c|}{1 - year } & \multicolumn{3}{|c|}{3 - year } & \multirow[b]{2}{*}{$d$} \\
\hline & $\begin{array}{l}\text { Follow-up } \\
(n=33)\end{array}$ & $\begin{array}{l}\text { No follow-up } \\
(n=9)\end{array}$ & $p$ & $d$ & $\begin{array}{l}\text { Follow-up } \\
(n=26)\end{array}$ & $\begin{array}{l}\text { No follow-up } \\
(n=15)\end{array}$ & $p$ & \\
\hline Age (yrs) & $71.12(6.52)$ & 70.44 (6.39) & .78 & .11 & $69.81(5.91)$ & $72.60(7.10)$ & .18 & .43 \\
\hline Gender ( $n$ female $)^{\gamma}$ & 21 & 7 & .43 & & 16 & 11 & .44 & \\
\hline Education (yrs) & $16.27(3.44)$ & $15.11(4.76)$ & .42 & .28 & $16.34(3.52)$ & $15.40(4.22)$ & .45 & .24 \\
\hline Health & $8.48(.97)$ & $8.61(1.11)$ & .74 & .12 & $8.58(.95)$ & $8.50(1.05)$ & .81 & .08 \\
\hline Visual acuity & $31.52(8.61)$ & $36.11(15.16)$ & .24 & .37 & 31.35 (9.33) & 34.67 (12.17) & .33 & .31 \\
\hline Short Blessed Test & $1.06(1.62)$ & $.44(1.33)$ & .30 & .42 & $.96(1.46)$ & $.93(1.83)$ & .96 & .02 \\
\hline BAI & $6.88(6.39)$ & 7.11 (5.95) & .92 & .04 & $7.31(6.72)$ & $6.53(5.60)$ & .71 & .13 \\
\hline Shipley & $36.70(3.27)$ & $34.67(5.41)$ & .16 & .45 & $36.31(3.40)$ & 36.00 (4.69) & .81 & .08 \\
\hline Go-No Go & $6.52(3.81)$ & $7.11(6.41)$ & .72 & .11 & $5.85(3.26)$ & 8.07 (5.87) & .13 & .47 \\
\hline Digit Symbol & 61.52 (12.85) & $54.56(11.84)$ & .15 & .56 & 62.15 (11.28) & $55.53(14.64)$ & .11 & .51 \\
\hline Letter Series & $9.67(4.42)$ & $8.44(4.28)$ & .46 & .28 & $10.12(4.35)$ & $8.27(4.43)$ & .20 & .42 \\
\hline ANT - Alerting & $28.32(42.41)$ & $31.97(30.70)$ & .81 & .10 & 34.07 (38.61) & $26.78(36.13)$ & .56 & .19 \\
\hline ANT - Orienting & $81.53(42.60)$ & $55.02(27.71)$ & .08 & .74 & $86.47(41.78)$ & $54.19(28.92)$ & $.01 *$ & .90 \\
\hline ANT - Exec control & $114.66(50.68)$ & $95.91(71.21)$ & .37 & .30 & $111.80(48.13)$ & $99.72(58.37)$ & .48 & .23 \\
\hline Task-switching Task & $16.76(236.86)$ & $39.93(115.14)$ & .78 & .12 & 38.56 (179.63) & .18 (276.59) & .59 & .16 \\
\hline
\end{tabular}




\section{LONG-TERM MAINTENANCE}

Note. Mean score with standard deviations presented in parentheses. BAI = Beck's Anxiety Inventory; Shipley = Shipley Vocabulary Test; ANT = Attention Network Test (Alerting, Orienting, Exec control = Executive control). Education was indexed by the average number of years of formal education; Health was indexed by a self-reported score out of 10; Visual acuity was indexed by the near visual acuity score from the Rosenbaum Visual Acuity Pocket Screener (score 20/--); Digit Symbol, Letter Series, and Shipley were scored by the average number of correct solutions; Average scores were displayed for BAI and Short Blessed Test. ; Go - No Go was scored by the average number of false alarms; ANT (Alerting, Orienting, and Exec control) and the Task-switching Task were scored by a difference score in ms.

${ }^{*} p<.05$. $^{\gamma}$ denotes the use of chi-square statistics; all other analyses used independent $t$ test; $d=$ Cohen's $d$ effect size calculation for between subjects.

\section{Materials and Procedure}

At each follow-up session, participants completed the Stroop task. The task consisted of four blocks: key-colour acquisition (Block 1; 40 trials), practice (Block 2; 24 trials), training (Block 3; 216 trials) and standard (Block 4; 144 trials). Each follow-up session implemented a similar format to the training sessions of the original study by Wilkinson and Yang (2012), except that no participants, regardless of the original feedback condition, received any feedback during Block 3 or 4. Responses were made by pressing colour-coded keys on a Stimulus-Response box.

In the original study, there were two counterbalance conditions in which the set of colours used for the Stroop task varied across sessions to minimize item-specific effects (for more details see Wilkinson \& Yang, 2012). At both follow-up sessions, participants completed a computerized version of the Stroop task that was adopted from session 1 of the original training study. At session 1 and the two follow-up sessions, the Stroop colours were as follows: blue, orange, green, and pink (counterbalance Condition 1), or pink, yellow, blue, and green (counterbalance Condition 2). To eliminate any itemspecific effects, at the 1-year follow-up session participants completed the Stroop task in the opposite counterbalance Condition than was assigned to them at session 1 of the original training. For example, if a participant was assigned to the colour set for counterbalance Condition 1 at original training, they were assigned to the colour set for 


\section{LONG-TERM MAINTENANCE}

counterbalance Condition 2 at the 1-year follow-up session or vice-versa. At the 3-year follow-up session, participants were assigned to the same colour set counterbalance Condition that was designated at original training. Following the example above, at the 3-year follow-up, this participant would be assigned to the colour set for counterbalance Condition 1 (same as in the original training).

The follow-up sessions were completed approximately one and three years after the final training session (i.e., session 6) of the original training (average 12.12 and 36.08 months, respectively). The average number of months between the 1-year and 3-year follow-up sessions was 23.96 months. A 2-month time window was allowed for each follow-up session to accommodate any scheduling conflicts.

\section{Results}

All statistical analyses were conducted in SPSS 20. The Stroop interference scores were calculated in RT for the standard block (Block 4) at each involved session to control for variations in the experimental trial procedure (e.g., feedback presented during original training). Only RTs for correct responses were included. RTs were trimmed such that any RT beyond 2.5 SD from the mean for each trial type at each follow-up session for each participant was excluded from the final data analysis. As a result, $2.34 \%$ of the RTs were deleted (for information on the outlier trimming of the original training data, see Wilkinson \& Yang, 2012).

The original training gains and long-term follow-up of Stroop inhibition training were explored using the Stroop RT interference scores (i.e., incongruent RT - neutral RT). Furthermore, for the analysis on the long-term maintenance effect, the effect sizes (Cohen's $d$ ) were calculated using a between-subjects calculation. This approach results 


\section{LONG-TERM MAINTENANCE}

in the smallest - and thus most conservative - effect size value, because the high correlation between repeated-measures is not used to reduce the error term (Dunlap, Cortina, Vaslow, \& Burke, 1996).

\section{Original Training Gains}

The analysis on the original training effect supported reliable training benefits i.e., significant session effects in either linear (suggesting incremental learning) or quadratic contrasts (suggesting saturation or ' $U$ ' shaped effects) for both follow-up samples. There was a significant linear effect of session for both those who completed the one-year follow-up $(n=33), F(1,32)=8.69, p=.006, \eta^{2}=.21$, as well as those who completed the three-year follow-up $(n=26), F(1,25)=7.48, p=.011, \eta^{2}=.23$ (see Figure 2). This indicates that the performance gains that were demonstrated in the original training study were also shown in the subset of participants that returned for the 1-year follow-up, as well as those who decided to also come back for the 3-year followup. 


\section{LONG-TERM MAINTENANCE}

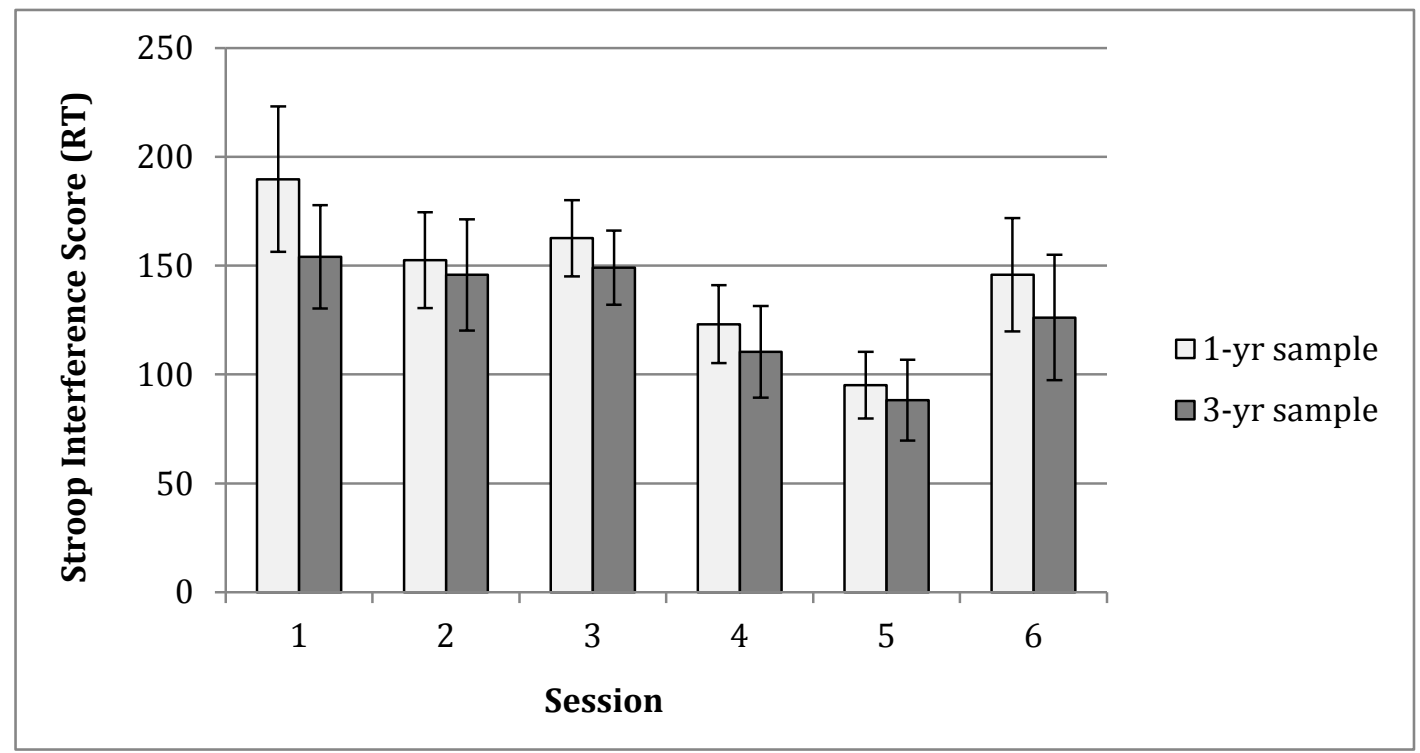

Figure 2. Mean RT Stroop interference scores (in ms) separately for those that completed the 1-year $(n=33)$ and 3-year follow-up sessions $(n=26)$ across the original six practice sessions. Error bars represent the standard error.

Visual inspection indicates that the best performance occurred at session 5 (not the final session - i.e., 6) for both samples. Furthermore, the first 3 sessions (week 1 of the original training) showed a similar performance level (no session effect for either the 1-year or 3-year follow-up samples, all ps $>.22$, and the last three sessions [week 2] showed consistently better performance than the average performance of week 1 ( $p=.001$ for both samples). So, to simplify the results, and best capture the learning effect, performance was averaged across week 1 and week 2 of the original training gains. Baseline performance was indexed by the average performance of week 1 of the original training, and the average performance of week 2 was used to represent the original training gains.

In order to evaluate the long-term maintenance of Stroop inhibition training, performance at week 2 of the original training was compared to each of the follow-up sessions separately (no difference supports the presence of maintenance effects). In 


\section{LONG-TERM MAINTENANCE}

addition, performance at the two follow-up sessions was compared to the performance of week 1 during original training to assess whether performance returned back to baseline levels. Given that there was some attrition between the two follow-up sessions ( $n=33$ for 1-year, $n=26$ for 3-year), these two sets of data were analyzed separately.

\section{Long-term Maintenance}

One-year follow-up. First, the long-term maintenance of the retest learning benefits one year following original training was evaluated. For the sample that returned for the 1-year follow-up session $(n=33)$, the RT interference scores were analyzed in a 3-way (session: week 1, week 2, 1-year follow-up) repeated-measures ANOVA. The analysis revealed a significant session effect, $F(2,64)=5.82, p=.005, \eta^{2}=.15$ (see Figure 3). Planned paired $t$ test were done to compare the original training gains (week 2) and baseline performance (week 1) to performance at the 1-year follow-up. The Stroop interference scores during week $2(M=121.35, S D=100.36)$ did not significantly differ from that at the 1 -year follow-up $(M=124.42, S D=99.56), p=.85, d=.03$. This supports the maintenance of the original gains one year after training. Furthermore, performance at the 1-year follow-up was significantly better (as indexed by smaller Stroop interference scores) than week 1 baseline performance $(M=168.28$, $S D=121.93), p=.01, d=.39$. This indicates that participants did not return to their baseline Stroop scores one year after the original training. 


\section{LONG-TERM MAINTENANCE}

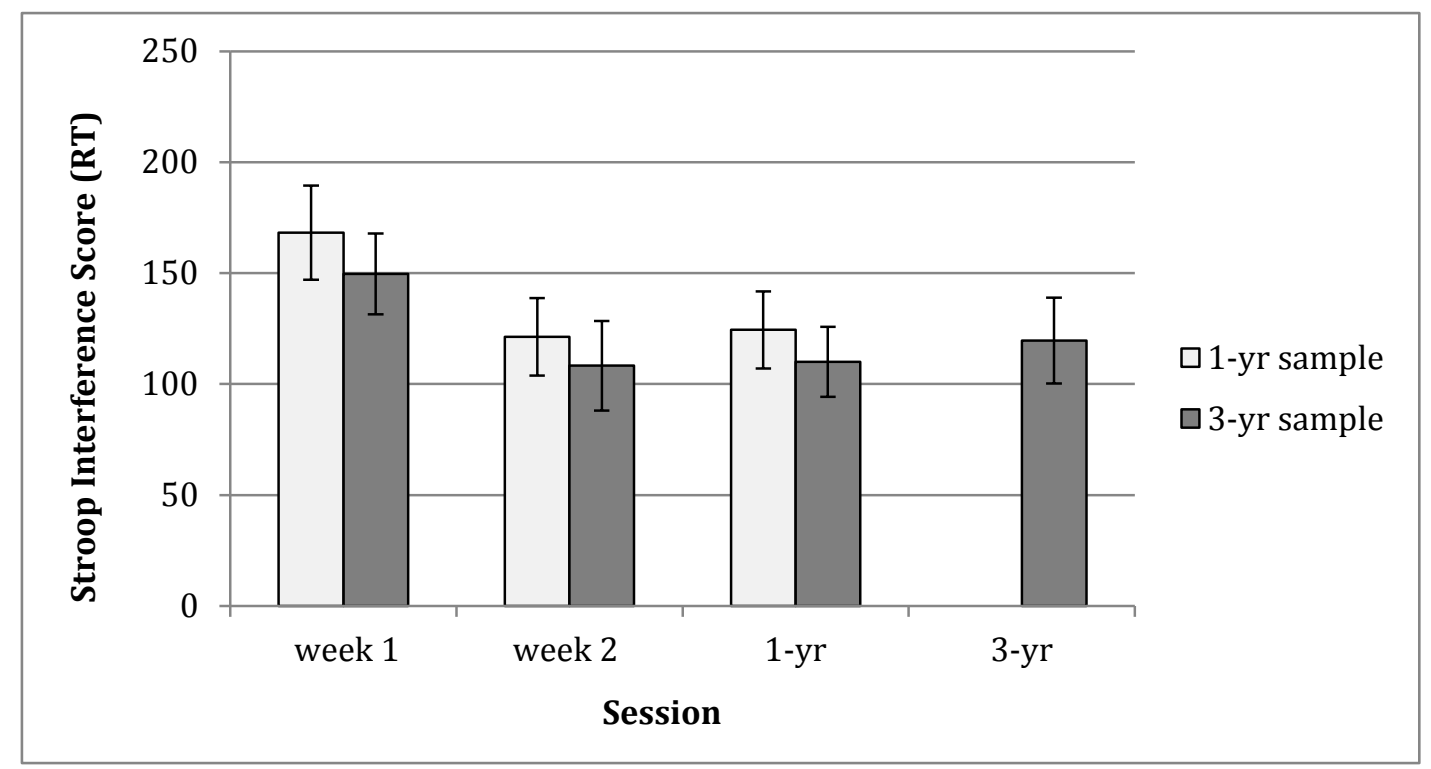

Figure 3. Mean RT Stroop interference scores (in ms) in week 1 and 2 of original training and the two follow-up sessions separately for those that completed the 1-year $(n=33)$ and 3-year follow-up sessions $(n=26)$. Error bars represent the standard error.

Three-year follow-up. For the sample that returned for the 3-year follow-up session (i.e., $n=26$ ), the RT interference scores were submitted to a 4-way (session: week 1, week 2, 1-year, 3-year) repeated-measures ANOVA. The session effect was marginally significant, $F(3,75)=2.59, p=.059, \eta^{2}=.094$ (see Figure 3). Planned $t$ tests were conducted to compare the performance at the two follow-up sessions (1-year and 3-year follow-up) with the original training gains (week 2) and baseline performance (week 1). Also, the interference scores at the two follow-up sessions were compared. Performance during week $2(M=108.26, S D=102.79)$ did not significantly differ from either follow-up session (1-year: $M=110.02$, $S D=80.46$; 3-year: $M=119.54$, $S D=98.64), p s>.51(d s \leq .11)$. This is in line with the above findings suggesting maintenance of training gains one year after training and further extends this to the 3-year follow-up session. In addition, performance at the 1-year follow-up was significantly better than at baseline $(M=149.63, S D=92.89), p=.05(d=.46)$ and trending in this 


\section{LONG-TERM MAINTENANCE}

direction at the 3-year follow-up, $p=.08(d=.31)$. Last, the comparison between the two follow-up sessions revealed equivalent performance, $p=.60(d=.11)$. Taken all together, these results suggest that training induced performance gains are maintained for one and even three years after training. In addition, performance at the two follow-up sessions is shown to be or tend to be better than baseline levels.

\section{Discussion}

Long-term maintenance of retest learning benefits in inhibition was assessed in healthy older adults at 1-year and 3-year follow-up points after a 2-week (3 sessions per week) Stroop training paradigm. The current data support the maintenance of training gains at both follow-up sessions. In other words, the reductions in RT Stroop interference scores, indexing better inhibition, achieved during the second week of a two-week training program was well maintained at both the 1-year and 3-year follow-up sessions. Furthermore, it was found that performance at the two follow-up sessions did not differ, and even show patterns of maintenance over and above the baseline performance levels one year after training, and are trending significant three years after training. To evaluate the effect of power to detect a statistical difference between performance at the 3-year follow-up and baseline, a post hoc power analysis was run using G*Power 3.1 software (Faul, Erdfelder, Lang, \& Buchner, 2007) for the difference between two dependent means (matched pairs) $t$ test. This analysis revealed a low statistical power of .69, given the sample size and design, to detect a medium effect (Cohen's $d=.5$ ), and very low statistical power of .33 to detect the small-to-medium effect (i.e., $d=.31$ ) that was present in the current data (using Cohen's effect size and power indexes; Cohen, 1988, 1992). This suggests that power issues, due to reduced sample size, are likely limiting our 


\section{LONG-TERM MAINTENANCE}

ability to find a significance difference between the 3-year follow-up and baseline (discussed further below).

Regardless, the current results suggest that older adults are able to maintain smallto-medium inhibition training gains over the periods of one and three years. These findings are in line with previous research (e.g., Dahlin et al., 2008a; Stigdotter Neely \& Bäckman, 1993); however, in the existing body of literature, the durability of executive function training gains are only explored for a short period of time - i.e., three months (e.g., Li et al., 2008) up to 18 months (e.g., Dahlin et al., 2008a). The current study adds to this literature by extending the maintenance of executive function training (using a Stroop task training program) to three years. Even though the current study shows performance at the 3-year follow-up does not return to baseline levels, with trending significance and a small-to-medium effect size, the maintenance of inhibition training gains may further benefit from the inclusion of booster sessions - a question for future research to address.

Although this follow-up study makes strong contributions to the literature, two limitations should be noted. First, the sample size at the 3-year follow-up session (i.e., $n=26)$ is small; however, I was restricted to the number of people who were willing to return for testing. Given the small sample size of the original training group (i.e., $n=42$ ), the final sample size at the 3-year follow-up was still quite impressive, with a $61.9 \%$ return rate. The second limitation is with respect to result generalizability. The current sample consists of a group of healthy, well-educated, and high functioning older adults, which may not be representative of the typical older adult population. Given this, generalizability of the current findings may be limited. 


\section{LONG-TERM MAINTENANCE}

Regardless of these limitations, the current data offer novel contributions to the literature by demonstrating that older adults are able to maintain gains in the restraint sub-function of inhibition for up to 3-years following initial training. Benefits in the ability to withhold automatic, but inappropriate responses given the current scenario can help to improve the daily lives of older adults. For example, if your doctor suddenly suggested that you stopped taking a certain medication - which you took regularly, every morning, having good restraint would minimize accidental ingestion, which may impact overall health and well-being. Or, consider this scenario: every morning you drive east to get from your home to work; but, one particular morning you need to drive west, because you have a dentist appointment. Having good restraint will minimize the likelihood of initially driving in the wrong direction - saving on time and reducing stress.

In conclusion, the current follow-up study adds to the literature by demonstrating long-term maintenance of retest learning in inhibition above baseline levels one and three years following original training. Remarkably, this effect was shown using a single session of the Stroop task at follow-up and without any booster sessions. 


\section{AGE DIFFERENCES}

Chapter 2. Age Differences in Three Sub-functions of Inhibition:

\section{Access, Deletion, and Restraint}

The primary goal of this study is to confirm the well-documented age differences in inhibition using six tasks commonly used in the literature to measure the three subfunctions of inhibition: Local-Global and Reading with Distraction (access), N-Back and Directed Forgetting (deletion), as well as Go - No Go and Stroop (restraint). Based on literature reviewed in the General Introduction, young adults are expected to outperform older adults in all three sub-functions. All six inhibitory tasks will be administered to each individual; therefore, the existence of expected age differences in inhibition across different tasks and/or sub-functions can be examined. Previous work by Feyereisen and Charlot (2008) found inconsistent age-related differences in various inhibitory tasks measuring the three sub-functions, with the expected deficits in deletion (Listening Span, Directed Forgetting) and restraint (Haylin Task, Stroop), but not in access (Word Problem Solving, Reading with Distraction). Their findings suggest that age-related deficits may not be uniform across the three sub-functions of inhibition. The current study will extend the findings of Feyereisen and Charlot (2008) by including three additional tasks: Local-Global, N-Back and Go - No Go.

\section{Method}

\section{Participants}

Fifty young adults (41 females, age range $=17-30$ years; $M=20.26, S D=3.42$ ) and 50 older adults (36 females, age range $=60-91$ years; $M=70.88, S D=8.30$ ) were included in the present study. The young adults were recruited from the undergraduate program at Ryerson University. The older adults were recruited from the community 


\section{AGE DIFFERENCES}

through the internal Ryerson Senior Participant Pool. The older adult sample (96\%) was part of the larger training study with three sub-functions of inhibition (see Chapter 3 of this dissertation), while the young adults were recruited solely for the purpose of comparison to performance at time 1 (pretraining session). All participants provided informed consent according to the REB at Ryerson University (see Appendix G for the consent agreement provided to the young adult sample and appendix details listed in Chapter 3 for information related to the older adult sample). Three participants (all older adults) presented with colour blindness, as measured with the Dvorine PseudoIsochromatic Plates (Dvorine Pseudo-Isochromatic Plates, 1953). The analyses for the colour dependent task (i.e., Stroop) were conducted with and without these individuals and the results were the same; so, their data were included in all of the analyses. All participants had reasonably normal near vision, with correction if applicable (range 20/20 - 20/50), as measured with the Rosenbaum near acuity pocket screener (Rosenbaum Pocket Vision Screener, 1986). No older adult participants showed dementia related cognitive impairment as all scored above the cut-off score of six on the Short Blessed Test (SBT; Katzman et al., 1983). Nine participants (all young adults) had severe anxiety, as indicated by a score higher than 25 on the Beck Anxiety Inventory (BAI; Beck, Epstein, Brown, \& Steer, 1988). In addition, twenty-six participants (17 young and 9 older) exceeded the cutoff score of 16 on the Centre for Epidemiological Studies of Depression Scale (CES-D; Radloff, 1977). Analyses were rerun excluding the participants that had severe anxiety and exceeded the CES-D cutoff score. The result patterns largely remained the same; so, these participants remained in the sample. The 


\section{AGE DIFFERENCES}

young and older adults differed in all baseline cognitive abilities and some demographic variables (see Table 2).

Table 2

Participant Demographic Characteristics and Cognitive Performance

\begin{tabular}{|c|c|c|c|c|}
\hline Characteristic & $\begin{array}{l}\text { Young } \\
(n=50)\end{array}$ & $\begin{array}{l}\text { Older } \\
(n=50)\end{array}$ & $p$-value & $d$ \\
\hline Age & $20.26(3.42)$ & $70.88(8.30)$ & $<.001 * *$ & 7.97 \\
\hline Gender ( $n$ female) ${ }^{\gamma}$ & 42 & 36 & .15 & \\
\hline Handedness ( $n$ right $)^{\gamma}$ & $46^{\mathrm{a}}$ & $44^{b}$ & .46 & \\
\hline Education (yrs) & $13.56(2.19)$ & $15.73(3.28)$ & $<.001 * *$ & .78 \\
\hline Visual acuity & 20.90 (2.19) & $26.70(6.03)$ & $<.001 * *$ & 1.28 \\
\hline Colourblindness $(n)^{\gamma}$ & 0 & 3 & .08 & \\
\hline Digit Symbol & $86.30(13.37)$ & $61.84(14.63)$ & $<.001 * *$ & 1.75 \\
\hline Letter Series & 13.80 (4.59) & $10.32(4.14)$ & $<.001 * *$ & .80 \\
\hline BAI (score) & $14.64(10.98)$ & $5.36(5.54)$ & $<.001^{* *}$ & 1.07 \\
\hline BAI ( $n$ severe) ${ }^{\gamma}$ & 9 & 0 & $.002 *$ & \\
\hline Shipley & $27.04(3.55)$ & $36.36(2.40)$ & $<.001 * *$ & 3.08 \\
\hline CES-D & $13.84(8.58)$ & $9.10(7.39)^{a}$ & $.004 *$ & .59 \\
\hline Short Blessed Test & $\mathrm{n} / \mathrm{a}$ & $0.74(1.38)$ & $\mathrm{n} / \mathrm{a}$ & \\
\hline
\end{tabular}

Note. Standard deviations are in parentheses. BAI = Beck's Anxiety Inventory; Shipley $=$ Shipley Vocabulary Test; CES-D = Centre for Epidemiological Studies of Depression Scale. Education was indexed by the average number of years of formal education; Visual acuity was indexed by the near visual acuity score from the Rosenbaum Visual Acuity Pocket Screener (score 20/--); Digit Symbol, Letter Series, and Shipley were scored by the average number of correct solutions; Average scores were displayed for BAI, CES-D, and Short Blessed Test. BAI ( $n$ severe) referred to the number of participants who scored above the cut-off score of 25.

${ }^{a}$ one participant did not complete the question/questionnaire

b one older adults responded 'right', but also identified several activities that were completed left-handed, so they were not included

${ }^{*} p<.05 .{ }^{* *} p<.001{ }^{\gamma}$ denotes the use of chi-square statistics; all other analyses used independent $t$ test; $d=$ Cohen's $d$ effect size calculation for between subjects.

\section{Design and Procedure}

All participants completed a consent form, and six computerized tasks (i.e., LocalGlobal, Reading with Distraction, N-Back, Directed Forgetting, Go - No Go and Stroop)

- counterbalanced across participants. They were also asked to complete some paper- 


\section{AGE DIFFERENCES}

pencil tasks to assess cognitive abilities (i.e., Letter Series, Digit Symbol, and Shipley Vocabulary Test), and to collect demographic information (i.e., background questionnaire). Young adults were given course credit for their participation. In contrast, older adults were compensated \$10/hour for their participation. All participants were debriefed, at the end of the session for young adults (see Appendix H), and at the end of the inhibition training study or (by the time they dropped out the training study) for older adults (see Chapter 3 for more details).

\section{Materials and Stimuli}

A 17-inch monitor PC computer was used for all of the computerized tasks. All participants were comfortably seated in a well-lit testing room at a viewing distance of approximately $60 \mathrm{~cm}$.

Cognitive tasks: Three sub-functions of inhibition. Both young and older adults completed six computerized tasks to assess the three sub-functions of inhibition two for each sub-function of inhibition. Each will be described in detail below.

Access. Local-Global and Reading with Distracting tasks were used to assess the access sub-function of inhibition.

Local-Global. The Local-Global task was modeled after Navon (1977) and Thomas et al. (2007). In this task, participants were presented with images that consisted of large numbers (global dimension; i.e., 1, 2, 3, 4) that were composed of small numbers (local dimension; i.e., 1, 2, 3, 4). Two out of the four numbers were considered as targets (e.g., '1' and '4') and two as control stimuli (e.g., '2' and '3') or vice versa ('2' and '3' as targets and ' 1 ' and ' 4 ' as control stimuli). Prior to each block, participants were instructed to attend to either the large number or the small numbers and to respond with 


\section{AGE DIFFERENCES}

one of two target number options (e.g., ' 1 ' or ' 4 '). The stimuli were approximately $6 \times 6 \mathrm{~cm}$ in size for the global dimension, and $0.5 \times 0.5 \mathrm{~cm}$ for the local dimension. Following, the classical Local-Global paradigm designed by Navon (1977) and replicated in later literature (e.g., Thomas et al., 2007), three types of trials were used: congruent, incongruent and neutral (see Figure 4).

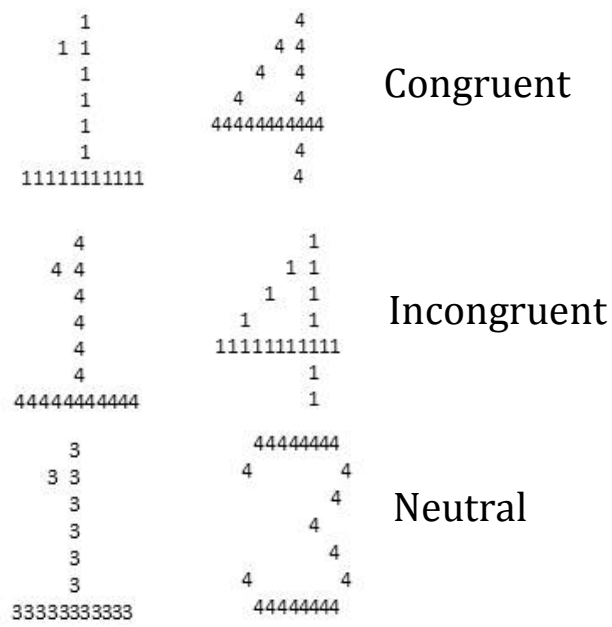

Figure 4. Sample of Local-Global stimuli for target numbers ' 1 ' or ' 4 ', with global dimension focus on the left and local dimension focus on the right.

For congruent trials, the local and global dimensions are matched - e.g., global number ' 1 ' made out of local numbers ' 1 ' (or a global ' 4 ' made out of local ' 4 's). In the case of congruent trials, the correct response is the same regardless of the instruction i.e., 'respond local' or 'respond global'. For incongruent trials, the local and global dimensions of the stimuli are conflicting in terms of the two target stimuli. For example, a global number ' 1 ' made out of local numbers '4' (or a global '4' made out of local ' 1 's). In the case of incongruent trials, the to-be-ignored dimension conflicts with the tobe-attended dimension, and the correct response changes depending on the given instruction. For neutral trials, the local and global dimensions are unmatched, but do not 


\section{AGE DIFFERENCES}

conflict in terms of the given targets. For example, using ' 3 ' as the control stimuli, in the global dimension focus, a global number ' 1 ' made out of local numbers ' 3 ' (respond ' 1 ') or, in the local dimension focus, a global number ' 3 ' made out of local numbers ' 4 ' (respond '4'; see Figure 4).

Instructions to focus on the local or global dimension of the stimuli were presented in a blocked design - counterbalanced across participants. The trials were pseudo-randomized, such that no more than three trials of the same type occurred consecutively. At each session, participants first completed two blocks of 12 practice trials (one for each dimension), followed by two experimental blocks (one for each dimension) of 72 intermixed trials each - 24 congruent, 24 incongruent and 24 neutral trials. Each trial began with a fixation-cross presented at the centre of the screen for 500 ms, which was replaced by a single Local-Global stimulus. The stimulus stayed on the screen until a response was made. During the practice blocks, feedback on accuracy and RT was provided for 2000 ms following each response. No feedback was given during the experimental blocks. Following a key press response, a blank screen was presented for 2000 ms, and then the next trial would begin. Responses were made by using the index finger of the left and right hand to depress the ' $z$ ' or ' $\{/\}$ ' keys labeled with the target numbers (e.g., ' 1 ' and '4'). The key assignment to targets was counterbalanced across participants.

The dependent variables of interest are interference scores calculated by the difference in RT between incongruent and congruent trials separately for the local and global dimensions (Kotchoubey et al., 1997; Navon, 1977). As mentioned in the General Introduction, young adults are expected to show a global precedence as indexed by faster 


\section{AGE DIFFERENCES}

overall RT and greater interference from the global as compared to the local dimension (Navon, 1977). In contrast, older adults are expected to show either an absence of global precedence, as indexed by equivalent dimension focus effects (Thomas et al., 2007) or a local precedence, as indexed by faster overall RT and greater interference from the local as compared to the global dimension (Slavin et al., 2002). In other words, access deficits are expected to be more global for young adults and local for older adults. In addition, greater interference overall is expected in older adults given their inhibitory deficits (Thomas et al., 2007).

Reading with Distraction. In the Reading with Distraction task (modified from Connelly et al., 1991), participants were instructed to read an individually presented short passage, describing a story (e.g., someone’s car falling apart and subsequently needing to take the bus), out loud. After reading the passage, the space bar was pressed, and four 6-option multiple-choice questions were presented one at a time. Participants selected their response by pressing the associated number on the top panel number keypad of the keyboard. They were told that accuracy was more important than being fast. For high distracting passages, one of the response options in the multiple-choice test was a to-beignored word. Selection of this multiple-choice response served as a measure of susceptibility to distracting material (discussed below).

There were two types of conditions: high distracting and low distracting. In both conditions, participants were told to read the italicized words out loud and to ignore the distracting materials that appeared in the display. The to-be-ignored, distracting material was either strings of Xs (low distracting condition) or not italicized words that were relevant to the story presented in the passage (high distracting condition). For example, in 


\section{AGE DIFFERENCES}

a passage about taking the bus after someone's car has fallen apart, the word 'train' was to-be-ignored. Each participant was presented with four passages in total (two high distracting and two low distracting), presented in alternating order (i.e., L-H-L-H or H-L-H-L). The presentation order was counterbalanced across participants.

There were two dependent variables of interest. The first was the reading speed of the high and low distracting passages. This quantifies the ability to keep irrelevant, distracting information outside one's focus of attention - with larger RT in high relative to low distracting conditions indexing poorer access. Second, the proportion of distractor intrusions in the multiple-choice response selection following the high distracting passages was evaluated. Specifically, a higher proportion of distractor intrusion represents worse access in that it suggests that more to-be-ignored information was allowed into working memory, which subsequently influenced passage comprehension.

Deletion. N-Back and Directed Forgetting were used to assess the deletion subfunction of inhibition.

N-Back. The N-Back task was modified from Braver et al. (1997). Participants completed four different N-back conditions: 0-Back, 1-Back, 2-Back and 3-Back. The conditions were presented in a blocked design and always in the same order (ascending from 0-Back to 3-Back).

Each block started with an alerting cue (*****) presented for $1000 \mathrm{~ms}$ followed by a blank screen for 500 ms. Each trial started with a stimulus presented at the centre of the screen for $500 \mathrm{~ms}$, which was replaced by a centrally presented fixation cross $(+)$ for 2000 ms. Participants were instructed to make their responses during the presentation of 


\section{AGE DIFFERENCES}

the fixation-cross. Feedback (i.e., 'Correct!', 'Incorrect', or 'No response detected') was presented at the end of each trial for $1500 \mathrm{~ms}$.

The stimuli were numbers ranging from 1 to 9 . In the 0-back condition, participants were instructed to indicate whether a pre-specified number (e.g., ‘5') appeared at all by pressing the key labeled 'TARGET,' if the target number was presented, and 'NON-TARGET', if any other number was presented. In the 1-Back condition, participants were instructed to press the key labeled 'TARGET', if the current number matched the one presented immediately before it (i.e., one trial back) and 'NONTARGET', if the current number did not match the one presented immediately before it.

Consider, for example, the following series of numbers: 1-2-2-1. In the 0-Back condition (e.g., using '5' as the 'TARGET'), all responses would be 'NON-TARGET', because no ' 5 ' was presented. In the 1-back condition, the correct responses would be 'NON-TARGET' for the first '1' because nothing came before it, 'NON-TARGET' for the ' 2 ' because it does not match the ' 1 ', 'TARGET' for the second ' 2 ', because it matches the stimulus that was presented immediately before it, and 'NON-TARGET' for the ' 1 ', because it does not match what came before. In the 2-Back condition, participants were instructed to press the 'TARGET' key if the number they see matches the one that was presented two numbers before. In the case of 1-2-2-1, the correct responses would be 'NON-TARGET' for all, because none of the numbers match what was presented two numbers prior. Last, for the 3-back conditions, participants were looking for 3-back matches. Again using the above example of 1-2-2-1, the correct responses would be 'NON-TARGET' for the first three stimuli and 'TARGET' for the last ' 1 ' because it matches what was presented three stimuli prior (i.e., the first ' 1 '). Subjects made their 


\section{AGE DIFFERENCES}

responses using the index fingers of their left and right hand. Key assignment (' $z$ ' or ‘/ for 'TARGET’ and 'NON-TARGET’) was counterbalanced across participants.

Participants first completed a 10 trial practice block for each condition (four practice blocks in total), followed by four 45-trial experimental blocks (one for each condition). Each experimental block contained nine target trials and 36 non-target trials presented in pseudorandom order. Nine of the non-target trials were foils - three for each of the non-tested experimental conditions (e.g., during the 2-back block there were three foils for each of the 0-back, 1-back and 3-back conditions). Non-target foils were trials that would have been considered a 'TARGET' trial, if presented in an alternate condition block. For example, if the series of numbers: 2-1-3-2 was presented in the 2-Back block, the second '2' would be considered a 3-Back foil trial, because it would have been a 'TARGET' trial had this number string been presented in the 3-Back block. Accuracy was based on the response to the currently presented trial. Overall accuracy for each condition (0-Back, 1-Back, 2-Back and 3-Back) was considered as the dependent variable of interest. Each condition required the removal of no longer relevant information for accurate completion of the task, except for the 0-Back block - which served as the control. Therefore, efficient deletion is indexed by higher accuracy scores - especially during the more inhibition dependent 2-Back and 3-Back conditions. Foil trials were included in this paradigm to increase interference and encourage engagement of the deletion sub-function of inhibition; however, the limited number of foil trials included in the current N-Back paradigm precludes any meaningful analyses of these data.

Directed Forgetting. The Directed Forgetting task was modeled after Sego, Golding, and Gottlob (2006; also see MacLeod, 1998, 1999). Seventy-two words were 


\section{AGE DIFFERENCES}

selected from the ANEW database (Bradley \& Lang, 1999) and divided into two lists of 36 words each (List A and List B). Lists A and B were further divided into three smaller lists of 12 words each (List 1, 2 and 3) and assigned to the to-be-remembered (TBR), tobe-forgotten (TBF) or NEW word conditions. Lists 1, 2 and 3 for both List A and List B were matched on critical psycholinguistic characteristics, including: valence, arousal, dominance, concreteness, frequency, length, meaning, and number of syllables. Lists A and $\mathrm{B}$ were counterbalanced to be used as the testing stimuli across participants. Furthermore, the assignment of List 1, 2 and 3 to the TBR, TBF or NEW word conditions was also counterbalanced across participants.

Each session consisted of three blocks: study, filler, and recognition. In the study block, participants were shown 24 words (12 TBR and 12 TBF words) - one at a time and instructed to remember some of them and forget others for a later memory test. The block began with a warning '*****' presented for $1000 \mathrm{~ms}$ at the centre of the screen. Each trial began with a single word presented in lower case letters at the centre of the screen for 5000 ms, followed by a 2000 ms blank screen. The blank screen was replaced with a $1000 \mathrm{~ms}$ cue instructing participants to either 'REMEMBER' (for TBR) or 'FORGET' (for TBF) the word for a later memory test. Following the cue, another blank screen was presented for 2000 ms before proceeding to the next trial. The TBR and TBF words were pseudo-randomized with no more than four words of the same condition appearing consecutively. Participants did not have to press any keys during the study block.

Following the study block, participants completed a filler task where they were presented with 50 completed math equations (e.g., $2+3=5$ ) and asked to indicate 


\section{AGE DIFFERENCES}

whether the equation was correctly completed or not. Half of the equations were correctly completed and half were not. Each trial began with a fixation-cross presented in the middle of the screen for $1000 \mathrm{~ms}$, followed by a math equation that remained on the screen until a response was made. Participants made a 'YES' or 'NO' judgment by pressing the '4' or ' 6 ' key on the number keypad. The 'YES'/'NO' key assignment was counterbalanced across participants.

Following the filler task, participants were given a forced response recognition task, where they were surprisingly asked to recognize all of the words presented during the study phase - regardless of cue. Specifically, they were instructed to respond 'old', if they remembered seeing the word during the study phase (i.e., regardless of the 'REMEMBER' or 'FORGET' instruction) and to respond 'new', if they did not remember seeing the word during the study phase. Thirty-six words were presented in total - all 24 words from the study phase plus 12 new words.

Each trial began with a single lower case word presented at the centre of the screen. The word remained on the screen until a response was given, and was then followed by a 1000 ms blank screen. Participants made their response by pressing the ' 1 ' or ' 3 ' key on the number keypad - marked with 'OLD' or 'NEW'. The key assignment was counterbalanced across participants.

There were several dependent variables of interests. First, the RT for 'new' responses to TBF (incorrect response) and NEW words (correct response) was explored, because the time it takes to reject a TBF word as 'new' provides an assessment of the deletion sub-function of inhibition. In particular, a slower 'new' response to a TBF word relative to a NEW word suggests that the former has not been effectively suppressed or 


\section{AGE DIFFERENCES}

removed from working memory, and, therefore, requires a longer time to reject. Zacks et al. (1996) followed this line of thinking and examined RT differences, but used 'no, this was not a word I had to remember' instead of 'new' responses. In addition, the hit rate (proportion of 'old' responses to OLD words) for TBR and TBF words was compared to examine the presence of a directed forgetting effect. In particular, greater hit rates for TBR relative to TBF words support a directed forgetting effect (Sego et al., 2006; Ullsperger, Mecklinger \& Müller, 2000; Zacks et al., 1996).

Restraint. Go-No Go and Stroop were used to assess the restraint sub-function of inhibition.

Go-No Go. The Go-No Go task was modeled after Donders (1868/1969). In this task, participants were shown a number stimulus - one at a time - on the computer screen. They were instructed to press the spacebar when a pre-specified 'Go' stimulus appeared and to withhold their response (i.e., not press the space bar), when a different, prespecified 'No Go' stimulus appeared. Number pairs of 1 and 9 or 4 and 8 were used as the stimuli for 'Go' and 'No Go' responses, respectively. The stimulus pair assignment, as well as the assignment to 'Go' and 'No Go' conditions within a given pair was counterbalanced across participants.

Participants completed 30 practice trials (20 'Go' and 10 'No Go'), followed by 200 experimental trials (150 'Go' and 50 'No Go'²). Each trial began with a fixation cross (+) presented at the centre of the screen for $1000 \mathrm{~ms}$, followed by a 'Go' or 'No Go’ stimulus presented centrally for 500 ms or terminated by a key press. The number of

\footnotetext{
2 One trial list (out of 4) mistakenly had 152 'Go' trials and 48 'No Go' trials. In this case, the proportion of false alarms was calculated by dividing the number of false alarms by 48 instead of 50 .
} 


\section{AGE DIFFERENCES}

hits (i.e., pressing the space bar on a 'Go' trial), misses (i.e., not pressing the space bar on a 'Go' trial), false alarms (i.e., pressing the space bar on a 'No Go' trial) and correct rejections (i.e., not pressing the space bar on a 'No Go' trial) were calculated. Following previous work (Allen et al., 1994; Falkenstein et al., 2002), the false alarm rate (i.e., the number of false alarms committed divided by the total number of 'No-Go' trials) was used to measure the restraint sub-function of inhibition, where a smaller false alarm rate represents greater capacity to withhold an automatic response.

Stroop. The Stroop task was modeled after (Wilkinson \& Yang, 2012 - adapted from Stroop, 1935) and included three types of trials: congruent (e.g., the word 'BLUE' printed in blue ink, respond blue), incongruent (e.g., the word 'BLUE' printed in green ink, respond green), and neutral (e.g., ' $\mathrm{XXXX}$ ' printed in blue ink, respond blue). Using different colour combinations, two versions of the task were created: blue, yellow, purple and brown (version 1) and green, pink, maroon, and orange (version 2). The version assignment was counterbalanced across participants. The structure of the Stroop task was the same as outlined in Chapter 1; therefore, only details where the two paradigms differ will be elaborated on here.

In this Stroop task, participants only completed three blocks of trials (in a fixedsequence): key-colour acquisition, practice, and experimental. The keys 'z', 'x', ‘’, and '/' were colour-coded to reflect the colours of the stimuli in the task. The colour-key assignment was consistent within the two version of the task. For version 1 , blue $=$ ' $z$ ', yellow $=$ ' $x$ ', purple $=\{$.$\} , and brown =\{/\}$. For version 2, green $=$ ' $z$ ', pink $=$ ' $x$ ', maroon $=\{$.$\} , and orange =\{/\}$. The very first two participants (both older adults) used keys on the number keypad to make their responses (i.e., '1', '2', '4', '5'); however, since 


\section{AGE DIFFERENCES}

the fingers could not be placed on these keys simultaneously, which could potentially affect RTs, this was modified to the letter keys mentioned above. All Stroop analyses were conducted with and without these participants; but, the results stayed the same, so their data were included in the analyses below.

The trials were pseudo-randomly presented, such that the same trial type (i.e., congruent, incongruent, and neutral) never appeared more than three times in a row, and there was no negative priming (i.e., the distracting colour word on the current trial was never the correct ink colour response on the subsequent trial; Little \& Hartley, 2000) or vice versa - i.e., the ink colour on the current trial was never the distracting colour word on the subsequent trial.

The first two blocks (key-colour acquisition and practice) followed the same structure as in Chapter 1; however, given the three block structure used here, the experimental block will briefly be described.

The third block was the experimental block and consisted of 216 trials (72 for each trial type: congruent, incongruent, and neutral). The trial procedure was the same as in the practice block, but without the feedback screen. Following previous literature (e.g., Dulaney \& Rogers, 1994; Milham et al., 2002; for a review see MacLeod, 1991) the dependent variables of interest were the Stroop interference scores calculated as the

difference RT and accuracy between incongruent and neutral trials. Smaller interference scores index better restraint. 


\section{AGE DIFFERENCES}

\section{Results}

All statistics were conducted using SPSS 20.0.

\section{Access}

Local-Global. Only correct trials were used in the current analysis (1.98\% of trials were deleted). Mean RTs were calculated for incongruent, congruent and neutral trials separately for the local and global blocks. RT data were trimmed such that any RT more than 2.5 SD away from the means and/or more than 5000 ms were deleted. This resulted in the exclusion of $3.18 \%$ of the trials. No RTs were signally premature (i.e., less than 200ms). Due to the program crashing, one participant skipped 40 global trials; so, the mean for this block was based on 32 trials (instead of 72). Also, one older adult participant responded incorrectly to all incongruent trials in the global block, probably due to misunderstanding of the instruction and/or the response key assignment, so the incongruent trial data in the global dimension for this participant were excluded from the analyses. Given the ceiling effects on accuracy (all means $\geq .97$ ), no analyses were conducted on accuracy (for raw scores see Tables 3). 


\section{AGE DIFFERENCES}

Table 3

Local-Global: Reaction Time, Accuracy and Interference Scores by Trial Type and Dimension Separately for Young and Older Adults

\begin{tabular}{|c|c|c|c|}
\hline Variable & $\begin{array}{l}\text { Young } \\
(n=50)\end{array}$ & $\begin{array}{l}\text { Older } \\
(n=49)^{\mathrm{a}}\end{array}$ & $d$ \\
\hline \multicolumn{4}{|l|}{ Accuracy: Local } \\
\hline Congruent & $.99(.02)$ & $1.00(.01)$ & .63 \\
\hline Incongruent & $.97(.04)$ & $.99(.03)$ & .57 \\
\hline Neutral & $.97(.05)$ & $1.00(.02)$ & .79 \\
\hline \multicolumn{4}{|c|}{ Accuracy: Global } \\
\hline Congruent & $.99(.02)$ & $1.00(.01)$ & .63 \\
\hline Incongruent & $.96(.07)$ & $.98(.03)$ & .37 \\
\hline Neutral & $.98(.03)$ & $1.00(.01)$ & .89 \\
\hline \multicolumn{4}{|c|}{ Reaction time: Local } \\
\hline Congruent & $491.03(91.91)$ & $648.48(263.57)$ & .80 \\
\hline Incongruent & $553.58(112.35)$ & $689.66(273.18)$ & .65 \\
\hline Neutral & $542.72(107.23)$ & $695.73(295.50)$ & .69 \\
\hline \multicolumn{4}{|c|}{ Reaction time: Global } \\
\hline Congruent & $467.60(75.55)$ & $674.60(243.57)$ & 1.15 \\
\hline Incongruent & 504.66 (83.67) & $772.59(285.46)$ & 1.27 \\
\hline Neutral & 484.88 (89.83) & 763.78 (289.89) & 1.30 \\
\hline \multicolumn{4}{|c|}{ Reaction time interference } \\
\hline Local & $62.55(59.94)$ & $41.18(53.61)$ & .38 \\
\hline Global & $37.06(41.72)$ & $97.99(80.87)$ & .95 \\
\hline
\end{tabular}

Note. Standard deviations are in parentheses. Local and Global = accuracy and reaction time (RT) performance during local/global dimension focus; Reaction time interference $=\mathrm{RT}$ incongruent $-\mathrm{RT}$ congruent.

${ }^{\mathrm{a}}$ The raw data presented reflect that used the statistical analyses. Due to missing data for the incongruent trials in the global dimension for one older adult, all analyses were conducted on $n=49 ; d=$ Cohen's $d$ effect size calculation for between subjects.

Overall $\boldsymbol{R T}$. The RTs were submitted to a 3 (trial type: congruent, incongruent, neutral) $\times 2$ (dimension: global vs. local) $\times 2$ (age: young vs. older) mixed model ANOVA. There was a main effect of trial type, $F(2,194)=82.63, p<.001, \eta^{2}=.46$. Post hoc paired $t$ tests revealed that the responses to the congruent trials $(M=569.51$, 


\section{AGE DIFFERENCES}

$S D=197.81)$ were significantly faster than those to the incongruent $(M=629.10$, $S D=217.71)$ and neutral trials $(M=620.69, S D=221.18), p s<.001$. The latter two, however, did not differ significantly, $p=.14$. All effects remained significant after Bonferroni corrections for multiple comparisons ( $p s<.02)$. There was also a main effect of age, $F(1,97)=28.70, p<.001, \eta^{2}=.23$. Unsurprisingly, young adults responded significantly faster $(M=507.41, S D=84.84)$ than did older adults $(M=707.47$, $S D=249.82$ ). These main effects were qualified by a significant trial type $\times$ age interaction, $F(2,194)=5.68, p=.004, \eta^{2}=.06$. Post hoc paired $t$ tests were conducted to compare the three different trial types separately for young and older adults. Young adults responded slower to incongruent trials $(M=529.12, S D=90.18)$ than to neutral trials $(M=513.80, S D=90.44)$, which in turn were responded to slower than congruent trials $(M=479.31, S D=80.30), p s \leq .006$. For older adults, incongruent trials $(M=731.12, S D=259.67)$ did not differ from neutral trials $(M=729.76, S D=259.78)$, $p=.89$, but both were slower than congruent trials $(M=661.54, S D=237.03), p s<.001$. All effects remained significant after Bonferroni corrections for multiple comparisons $(\operatorname{six} ; p s \leq .008)$

There was also a significant dimension $\times$ age interaction, $F(1,97)=11.33$, $p=.001, \eta^{2}=.11$. Young adults responded faster to the global $(M=485.71, S D=79.62)$ than to the local dimension $(M=529.11, S D=99.70), p<.001$. Older adults, in contrast, showed the opposite pattern, with a faster average response to the local ( $M=677.96$, $S D=270.30)$ than to the global dimension $(M=736.99, S D=270.18), p=.05$. The latter, however, did not stay significant after Bonferroni correction for multiple (two) comparisons $(p<.03)$. Taken together, this suggests that young, but not older, adults 


\section{AGE DIFFERENCES}

showed a global precedence effect. Last, there was a significant higher order three-way interaction of trial type $\times$ dimension $\times$ age, $F(2,194)=8.38, p<.001, \eta^{2}=.08$. Post hoc paired $t$ tests were conducted to compare the three trial types within each dimension separately for young and older adults. After applying Bonferroni corrections for multiple (12) comparisons $(p \leq .004)$, young adults showed neutral > congruent in the local block, as well as incongruent $>$ neutral in the global block, $p \leq .002$. Older adults showed neutral $>$ congruent in the global block, $p<.001$ (see Table 3 for raw RTs). Importantly, both young and older adults showed slower RT for incongruent relative to congruent trials in both dimensions (all ps <.001).

$\boldsymbol{R T}$ interference scores. In order to further investigate the existence of age difference in interference from the local or global dimensions, RT interference scores (incongruent RT - congruent RT) were calculated separately for the local and global blocks and submitted to a 2 (dimension: local vs. global) $\times 2$ (age: young vs. older) mixed model ANOVA. The main effect of dimension was approaching, but did not reach significance, $F(1,97)=2.88, p=.09, \eta^{2}=.03$. There was, however, a significant main effect of age, $F(1,97)=6.17, p=.02, \eta^{2}=.06$, with older adults showing greater interference scores overall $(M=69.59, S D=43.09)$ as compared to young adults $(M=49.81, S D=35.86)$. Importantly, there was also a significant dimension $\times$ age interaction, $F(1,97)=19.89, p<.001, \eta^{2}=.17$. In particular, young adults showed larger interference during local focus $(M=62.55, S D=59.94)$ than global focus $(M=37.06$, $S D=41.72$ ), $p=.02$, while the opposite was true for older adults, who showed larger global $(M=97.99, S D=80.87)$ than local focus interference effects $(M=41.18$, $S D=53.61$ ), $p=.001$ (see Figure 5; for raw scores see Table 3). All $p$ s remained 


\section{AGE DIFFERENCES}

significant after Bonferroni corrections for multiple comparisons (two; $p$ s < .03). Looking at this interaction from a different perspective, older adults showed significantly larger interference as compared to young adults in the global dimension $(p<.001, d=.95)$; however, they tended to show smaller interference in the local dimension focus $(p=.07$, $d=.38$ ). This suggests that young adults were more disrupted from the global dimension (quantified by the RT interference score during local focus), whereas older adults were more disrupted from the local dimension (quantified by the RT interference score during global focus).

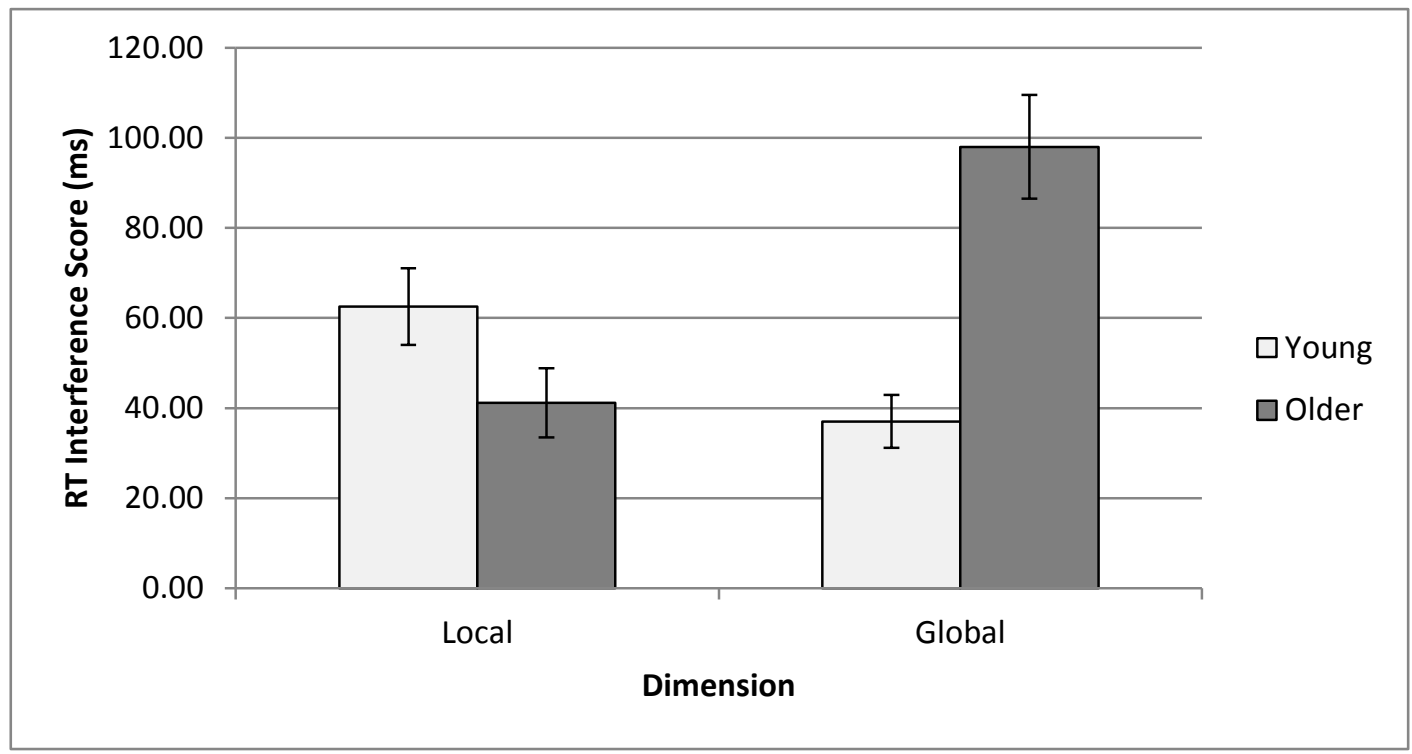

Figure 5. RT interference scores during local and global dimension focus separately for young and older adults. Error bars represent the standard error.

Reading with Distraction. Each participant read four passages in total: two high distracting and two low distracting. The RT for each condition was taken as the average of the two passages. Due to the program crashing, one participant only read one high distracting passage; so, their average for this condition was based only on a single data point. All RTs were within the range of 2.5 SDs from the mean for each condition; therefore, no further trimming was applied. In addition, the proportion of distractor 


\section{AGE DIFFERENCES}

intrusions in the multiple-choice task was assessed. It is important to note that this occurrence was only possible in the high distracting condition, because the to-be-ignored distractors were words instead of strings of Xs as in the low distracting condition.

Passage reading speed. Passage reading RT (in seconds) was submitted to a 2 (condition: high vs. low) $\times 2$ (age: young vs. older) mixed model ANOVA. There was a main effect of condition, $F(1,98)=214.34, p<.001, \eta^{2}=.69$. The high distracting passages $(M=86.35, S D=29.68)$ took longer to read as compared to low distracting passages $(M=62.35, S D=16.89)$. There was also a main effect of age, $F(1,98)=24.98$, $p<.001, \eta^{2}=.20$, with older adults taking longer in overall reading speed $(M=84.52$, $S D=25.53)$ than young adults $(M=64.18, S D=13.29)$. The interaction was marginally significant, $F(1,98)=3.11, p=.08, \eta^{2}=.03$, and the results were in the right direction. Specifically, older adults showed a larger difference in reading speed between the high and low distracting conditions $(M=26.89, S D=19.80)$ than young adults $(M=21.11$, $S D=12.07$ ). Cohen’s $d=.35$, represents a small-medium effect size (Cohen, 1988; 1992; see Table 4).

Multiple-choice (overall accuracy). The proportion of accurate responses in the multiple-choice overall task was submitted to a 2 (condition: high vs. low) $\times 2$ (age: young vs. older) mixed model ANOVA. There was a significant main effect of condition, $F(1,98)=53.22, p<.001, \eta^{2}=.35$, with reduced accuracy in the high $(M=.77$, $S D=.13)$ as compared to the low distracting condition $(M=.89, S D=.10)$. Furthermore, there was a significant main effect of age, $F(1,98)=5.69, p=.02, \eta^{2}=.06$, with greater overall accuracy in young $(M=.85, S D=.09)$ versus older adults $(M=.81, S D=.09)$. 


\section{AGE DIFFERENCES}

There was, however, no significant interaction, $F(1,98)=1.09, p=.30, \eta^{2}=.01$ (see Table 4).

Multiple-choice (distractor intrusions). The proportion of to-be-ignored distractor intrusions were submitted to an independent $t$ test. This analysis revealed that older adults committed significantly more to-be-ignored distractor intrusions $(M=.21$, $S D=.10$ ) during the multiple-choice component of the task as compared to young adults $(M=.16, S D=.10), p=.01($ see Table 4$)$.

\section{Table 4}

Reading with Distraction: Passage Reading Speed, Multiple Choice Performance, and Proportion of Distractor Intrusions Separately for Young and Older Adults

\begin{tabular}{llll}
\hline Variable & $\begin{array}{l}\text { Young } \\
(n=50)\end{array}$ & $\begin{array}{l}\text { Older } \\
(n=50)\end{array}$ & $d$ \\
\hline Passage reading speed & & & \\
Low distraction & $53.62(96.92)$ & $71.08(18.09)$ & .25 \\
High distraction & $74.74(18.22)$ & $97.97(34.24)$ & .85 \\
Distraction score & $21.11(12.07)$ & $26.89(19.80)$ & .35 \\
Multiple-choice performance & & & .19 \\
Low distraction & $.90(.09)$ & $.88(.12)$ & .48 \\
High distraction & $.80(.13)$ & $.74(.12)$ & .50 \\
Distractor intrusion rate & $.16(.10)$ & $.21(.10)$ & .39 \\
\hline
\end{tabular}

Note. Standard deviations are in parentheses. Passage reading speed denoted in seconds and multiple-choice performance denoted in proportion of accurate responses for low and high distracting conditions. Distraction score was indexed by the difference in reading speed between high and low distracting conditions; distractor intrusion rate was indexed by the proportion of tobe-ignored words selected during the multiple-choice task for the high distracting condition only. $d=$ Cohen's $d$ effect size calculation for between subjects.

\section{Deletion}

N-Back. First, all 'no response detected' trials (2.83\%) were removed from the dataset. Then the proportion of accurate responses was calculated separately for each 


\section{AGE DIFFERENCES}

condition (0-Back, 1-Back, 2-Back, and 3-Back). One participant chose to stop responding during the 3-Back block, so the mean of this block was calculated based on a smaller subset of trials. In addition, the E-prime program crashed several times (inconsistently), and thus resulted in fewer generated trials in the 2-Back and 3-Back block for two participants. For these two individuals, the means for these blocks were calculated based on the generated trials only. For the participant where the program crashed during the 2-Back block, no 3-Back trials were completed at all; so, this remained a missing data point.

Overall accuracy. Overall accuracy scores were submitted to a 2 (age: young vs. older) $\times 4$ (condition: 0-Back, 1-Back, 2-Back, 3-Back) mixed model ANOVA. This analysis revealed a significant main effect of age, $F(1,97)=12.22, p=.001, \eta^{2}=.11$. Specifically, young adults showed significantly greater accuracy $(M=.91, S D=.05)$ as compared to older adults $(M=.88, S D=.05)$. There was also a main effect of condition, $F(3,291)=197.49, p<.001, \eta^{2}=.67$. Accuracy was highest in the 0-Back, followed by 1-Back and 2-Back conditions, with lowest accuracy in the 3-Back condition, all ps $<.001$. All effects remained significant after adjusting using Bonferroni correction ( $p$ s $<.01)$. The two main effects were qualified by a significant age $\times$ condition interaction, $\quad F(3,291)=3.61, p=.01, \eta^{2}=.04$. Post hoc independent $t$ tests were conducted comparing the two age groups for each condition separately. All significance levels were adjusted for the number of comparisons (four) using Bonferroni correction $(p \leq .01)$. Given this criterion, the age effect was significant during the 3-Back block, $p=.001$; and was approaching significance for the 2-Back block, $p=.019$ (see Figure 6). No other effects were significant, $p \mathrm{~s} \geq .09$. 


\section{AGE DIFFERENCES}

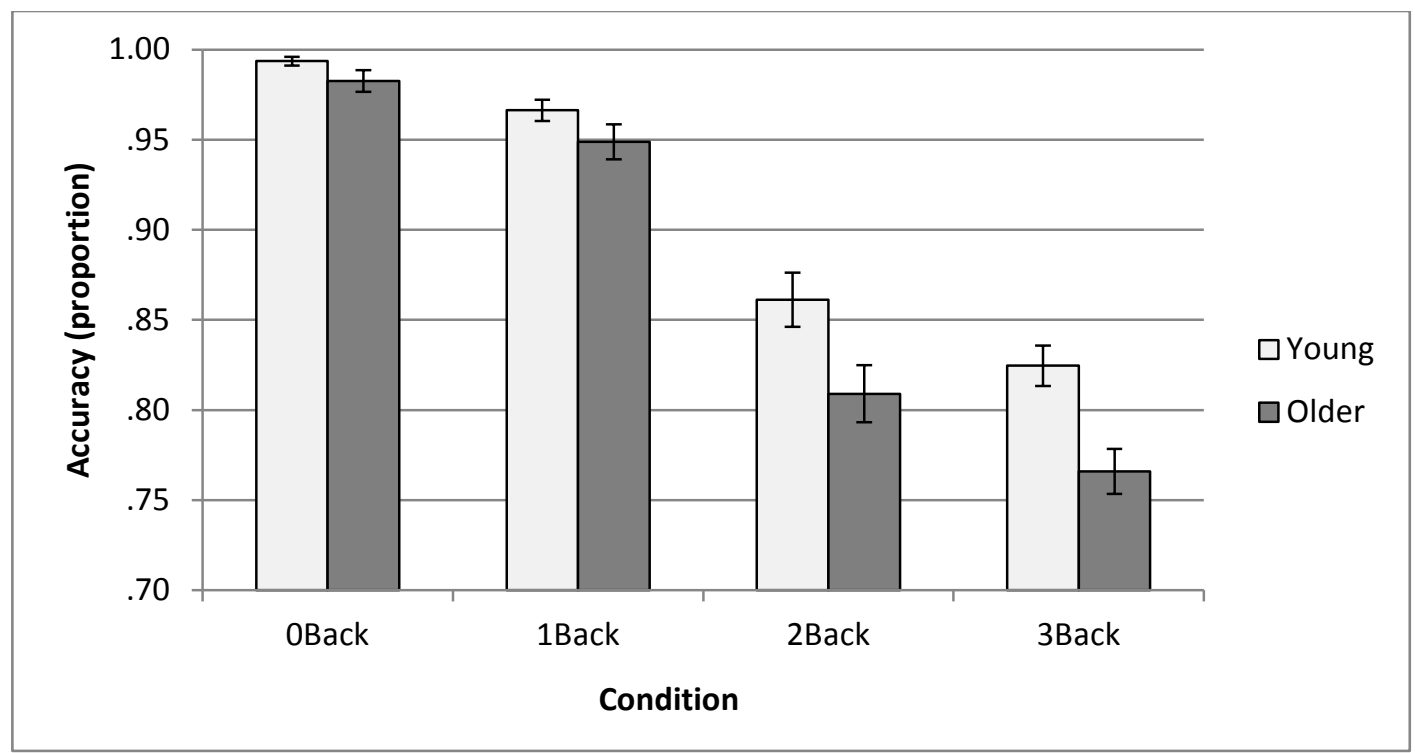

Figure 6. Proportion of accurate responses in N-Back across four conditions separately for young and older adults. Error bars represent the standard error.

Directed Forgetting. RT data (collected during the recognition task) was calculated separately for correct and incorrect responses to TBR, TBF and NEW words. The response time data were trimmed such that RTs greater than 2.5 SDs away from the cell mean for each word type - for incorrect and correct responses - were removed. Given this criterion, $3.97 \%$ of the trials were deleted. First, RT for 'new' responses to TBF (i.e., incorrect response) and NEW words (i.e., correct response) was examined. Following the RT analysis, hit rates (i.e., proportion of 'old' responses to OLD words) were also examined for TBR and TBF words.

Overall RT. Overall RT for 'new' responses to TBF words and NEW words were submitted to a 2 (word type: TBF vs. NEW) $\times$ age (2: young vs. older) mixed model ANOVA. Since only incorrect RTs to TBF words were included in this analysis, participants who never responded 'new' to TBF words were excluded. Therefore, this RT analysis only includes data from 36 young and 34 older adults. 


\section{AGE DIFFERENCES}

There was a main effect of word type, $F(1,68)=47.32, p<.001, \eta^{2}=.41$. In particular, it took longer to respond to TBF words $(M=1443.00, S D=554.64)$ than to NEW words $(M=1134.74, S D=357.01)$, suggesting that rejecting a TBF item as 'new' took a longer time than accepting a NEW item as 'new'. There was also a main effect of age, $F(1,68)=35.98, p<.001, \eta^{2}=.35$. Overall, young adults $(M=1049.54$, $S D=298.23)$ responded faster than older adults $(M=1542.28, S D=385.77)$. Importantly, there was also a significant interaction between word type and age, $F(1,68)$ $=8.21, p=.006, \eta^{2}=.11$. To tease apart this interaction, an independent $t$ test was used to examine the effect of age on the RT difference score between TBF and NEW words. Greater differences were revealed for older $(M=441.88, S D=436.95)$ than young adults $(M=182.06, S D=315.42), p=.006$. This suggests that older adults had a harder time removing TBF words from working memory, and thus took a longer time to reject them as 'new' during the recognition memory test (see Figure 7 and Table 5).

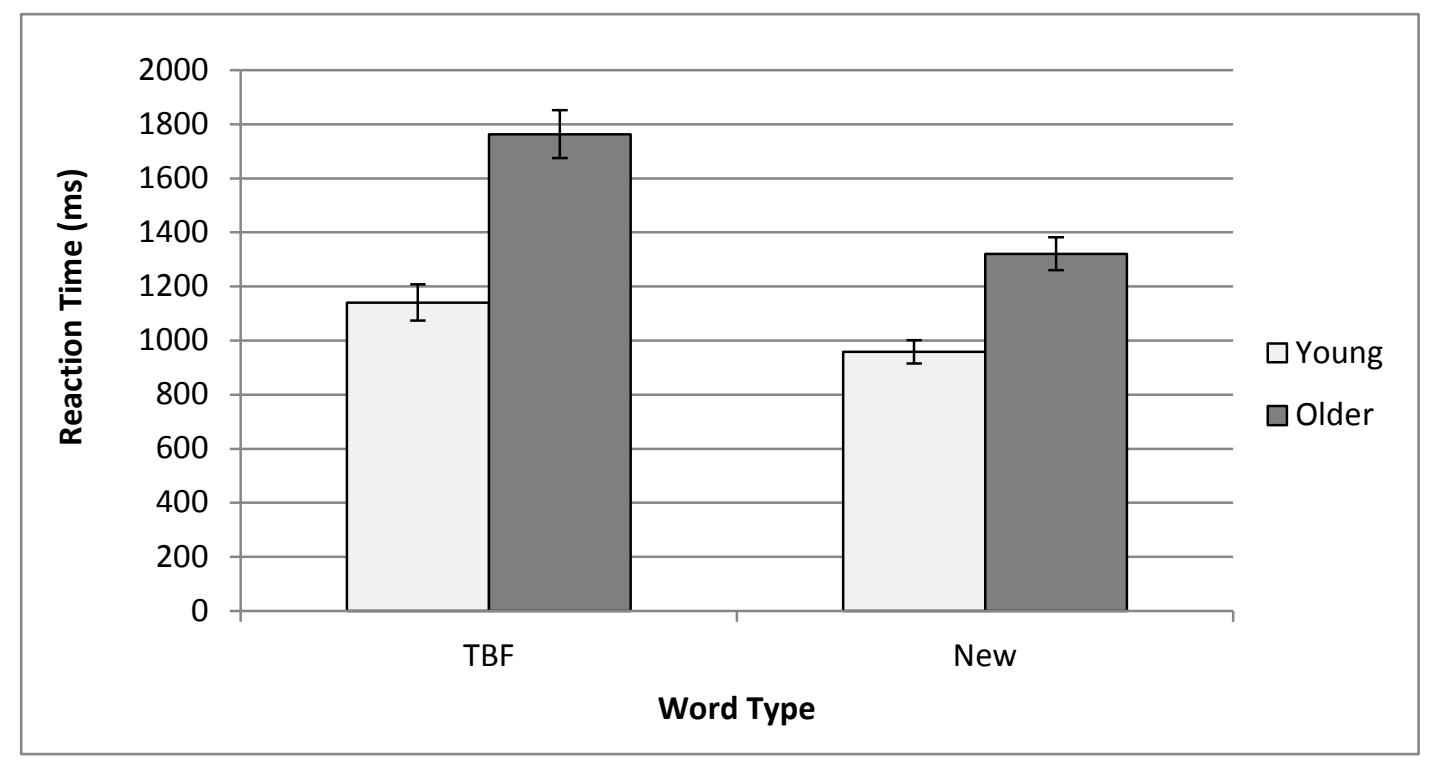

Figure 7. Mean RT in Directed Forgetting for 'new' response to TBF and NEW words separately for young $(n=36)$ and older adults $(n=34)$. Error bars represent the standard error. 


\section{AGE DIFFERENCES}

Hit rate. One young participant did not respond 'old' to any of the TBF words; so, these data were removed from the analysis below. Hit rates for TBR and TRF items were submitted to a 2 (word type: TBR vs. TBF) $\times 2$ (age: young vs. older) mixed model ANOVA. There was a significant effect of word type, $F(1,97)=54.22, p<.001$, $\eta^{2}=.36$. In particular, the proportion of TBR hits $(M=.88, S D=.13)$ was significantly higher than TBF hits $(M=.75, S D=.20)$, which confirms the presence of a directed forgetting effect. No other effects were significant (all other $p s$ >.76).

Table 5

Directed Forgetting: Mean Reaction Time, Hit Rate and False Alarm Rate for Young and Older Adults

\begin{tabular}{llll}
\hline Variable & $\begin{array}{c}\text { Young } \\
(n=50)\end{array}$ & $\begin{array}{l}\text { Older } \\
(n=50)\end{array}$ & $d$ \\
\hline Reaction time & & & \\
TBF ('new') & $1140.57(400.74)^{\infty}$ & $1763.22(517.16)^{\Psi}$ & 1.35 \\
NEW ('new') & $958.52(258.91)^{\infty}$ & $1321.34(354.46)^{\Psi}$ & 1.17 \\
Hit rate & $.89(.10)^{\S}$ & & .08 \\
TBR & $.75(.20)^{\S}$ & $.88(.15)$ & .00 \\
TBF & & $.75(.20)$ & .70 \\
False alarm rate & $.13(.11)^{\S}$ & $.06(.09)$ & \\
NEW & & & \\
\hline
\end{tabular}

Note. Standard deviations are in parentheses. Reaction time (in ms) for TBF words incorrectly identified as 'new' and NEW words correctly identified as 'new'; Hit rate denoted as the proportion of TBR or TBF words correctly identified as 'old'; False alarm rate denoted as the proportion of NEW words incorrectly identified as 'old'.

${ }^{\Psi} n=34 .{ }^{\infty} n=36 .{ }^{\S}=n=49$.

$d=$ Cohen's $d$ effect size calculation for between subjects.

Even though no age difference in the hit rates was found, post hoc independent $t$ tests revealed that young adults committed significantly more false alarms $(M=.13$, 


\section{AGE DIFFERENCES}

$S D=.11)$ than older adults $(M=.06, S D=.09), p=.003$. Given this difference, sensitivity analyses were considered, but could not be evaluated. In sensitivity analyses, it is assumed that a hit (i.e., responding 'old' to an OLD item) is composed of two types of responses - one where items are truly recognized as 'old' and lucky guesses. The false alarm rate (responding 'old' to a NEW item) is used to index guessing. A sensitivity analysis using Snodgrass and Corwin's (1988) discrimination index $\left(\mathrm{P}_{\mathrm{r}}\right)$ is calculated by subtracting false alarms from hit rates, and thus index recognition after correcting for guessing. Given the structure of the Directed Forgetting paradigm used here, only a single false alarm rate could be determined; therefore, calculating $\mathrm{P}_{\mathrm{r}}$ scores would be redundant with the analyses above and so were not considered further.

\section{Restraint}

Go - No Go. False alarm rates were submitted to an independent $t$ test comparing young and older adults, but no significant differences were revealed, $t(98)=.452$, $p=.652$ (see Table 6 for details).

Close inspection of the raw data revealed some very high miss rates (i.e., not pressing the space bar on a 'Go' trial), but the miss rate did not differ between the two age groups $(t=-.26, p=.80)$. This indicates that perhaps due to a computer or programming issue, not all space bar presses were being recorded. To address this issue, individuals with miss rates of $30 \%$ or higher ( $n=15 ; 7$ young and 8 older) were flagged and excluded from data prior to this follow-up analysis. The results, however, remained the same $t(83)=.429, p=.669$ (see Table 6 ), and no age differences were found in the proportion of false alarms committed during the Go - No Go task. 


\section{AGE DIFFERENCES}

Table 6

Go - No Go: False Alarm Rate for Full and Reduced Sample Separately for Young and Older Adults

\begin{tabular}{clll}
\hline Task & $\begin{array}{l}\text { Young } \\
(n=50)\end{array}$ & $\begin{array}{l}\text { Older } \\
(n=50)\end{array}$ & $d$ \\
\hline False alarm rate & & & \\
Full sample $(n=100)$ & $.018(.016)$ & $.016(.021)$ & .11 \\
Reduced sample $(n=85)$ & $.020(.016)^{\Omega}$ & $.018(.023)^{\Phi}$ & .10 \\
\hline
\end{tabular}

Note. Standard deviations are in parentheses. Reduced sample represented the participants with miss rates less than .30. False alarm rate was denoted as the proportion of times the space bar was hit on a 'No Go' trial.

${ }^{\Phi} \mathrm{n}=42 .{ }^{\Omega} \mathrm{n}=43$.

$d=$ Cohen's $d$ effect size calculation for between subjects.

Stroop. Only correct trials were used in the current analysis (4.47\% of trials were removed for being incorrect). RTs were calculated separately for each trial type and were trimmed by removing any RT more than 2.5 SDs away from the mean of each cell. As a result, $2.70 \%$ of the trials were removed. Analyses on the accuracy data are reported first, followed by that of the RT data.

Overall accuracy. Overall proportion of accurate responses was submitted to a 3 (trial type: congruent, incongruent, neutral) $\times 2$ (age: young vs. older) mixed model ANOVA. There was a significant effect of trial type, $F(2,196)=19.73, p<.001$, $\eta^{2}=.17$. Post hoc paired $t$ test revealed a lower accuracy on incongruent trials $(M=.93$, $S D=.10)$ than on both congruent $(M=.97, S D=.04)$ and neutral trials $(M=.97$, $S D=.04), p s<.001$. The latter two did not differ, $p=.26$. All significant effects remained after Bonferroni corrections. There was also a main effect of age, $F(1,98)=16.96, p<.001, \eta^{2}=.15$. Surprisingly, young adults $(M=.94, S D=.05)$ 


\section{AGE DIFFERENCES}

committed significantly more errors than the older adults $(M=.97, S D=.04)$. There was, however, no significant interaction, $F(2,196)=1.17, p=.31, \eta^{2}=.01$. Curiously, visual inspection of the raw data (see Figure 8) shows greater differences between incongruent and neutral trials for the young adults as compared to the older adults (also see Table 7). This potential age effect was further explored below with an examination of interference scores.

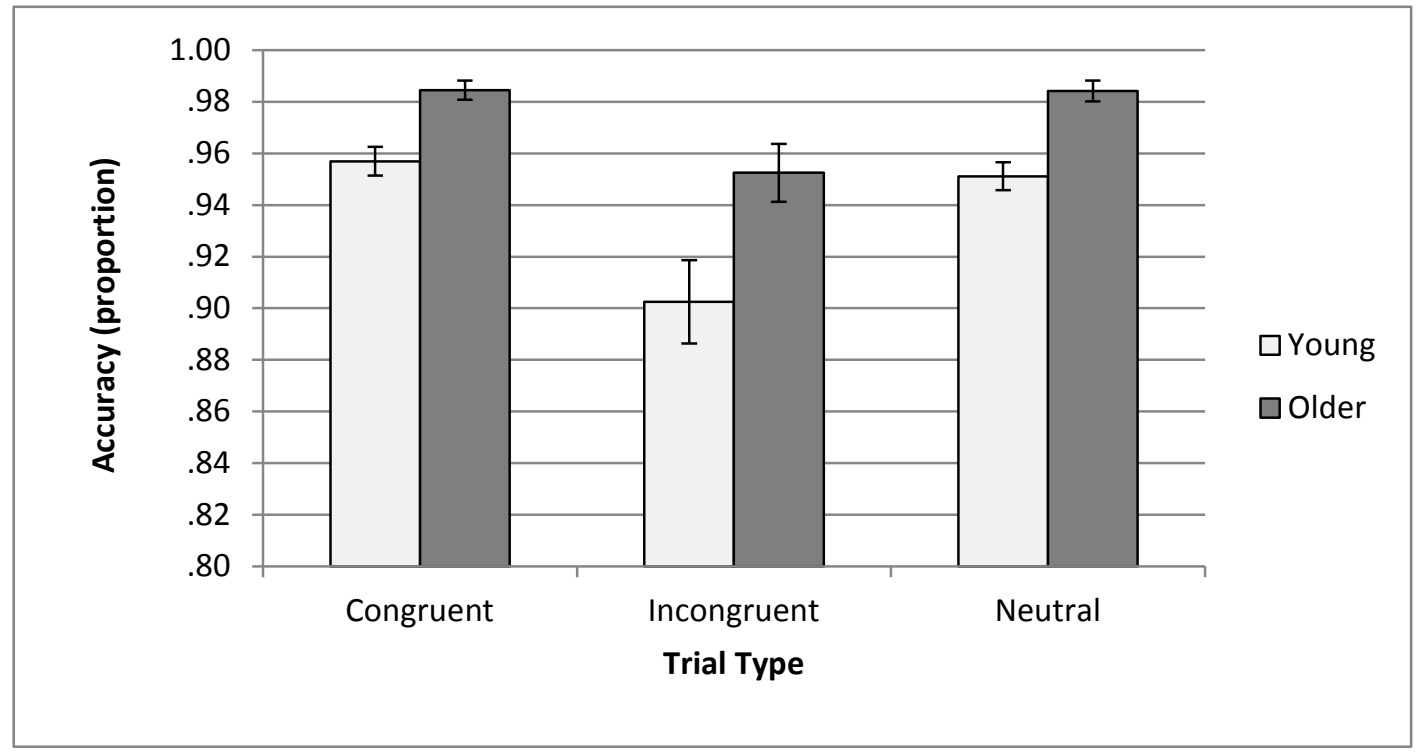

Figure 8. Proportion of accurate responses in Stroop for congruent, incongruent, and neutral trials separately for young and older adults. Error bars represent the standard error.

Stroop interference (accuracy). Stroop interference scores were calculated by subtracting the accuracy of incongruent trials from that of neutral trials. An independent $t$ test on these scores showed no age differences, $p=.36$, indicating equivalent age effect with regards to interference as measured by accuracy performance.

Overall RT. Overall RT was submitted to a 3 (trial type: congruent, incongruent, neutral) $\times 2$ (age: young vs. older) mixed model ANOVA. There was a significant effect of trial type, $F(2,196)=165.95, p<.001, \eta^{2}=.63$. Post hoc paired $t$ test revealed that RT on incongruent trials $(M=939.40, S D=343.10)$ was significantly slower than 


\section{AGE DIFFERENCES}

congruent $(M=782.59, S D=260.79)$ and neutral trials $(M=790.64, S D=247.75)$, $p s<.001$. The latter two were trending, but did not reach significance, $p=.08$. The significant differences remained after using Bonferroni corrections for three comparisons (ps < .02) and suggest that when the ink colour and colour word are mismatched (incongruent), RT was significantly longer.

There was also a main effect of age, $F(1,98)=36.82, p<.001, \eta^{2}=.27$. In particular, young adults $(M=691.50, S D=121.89)$ responded significantly faster than older adults $(M=983.59, S D=317.79)$. Both main effects were qualified by a significant interaction between trial type and age, $F(2,196)=12.14, p<.001, \eta^{2}=.11$. To tease apart this interaction, paired $t$ tests to compare RTs of all three trial types were conducted for each age group separately. Both age groups showed significantly slower RT for incongruent as compared to congruent and neutral trials, $p s<.001$, while the latter two did not differ significantly, $p s>.11$. Visual inspection of the raw data (see Figure 9) shows greater differences between incongruent and neutral trials for older as compared to young adults, which helps to explain the age $\times$ trial type interaction (also see Table 7). This potential age effect was further explored below with Stroop interference scores. 


\section{AGE DIFFERENCES}

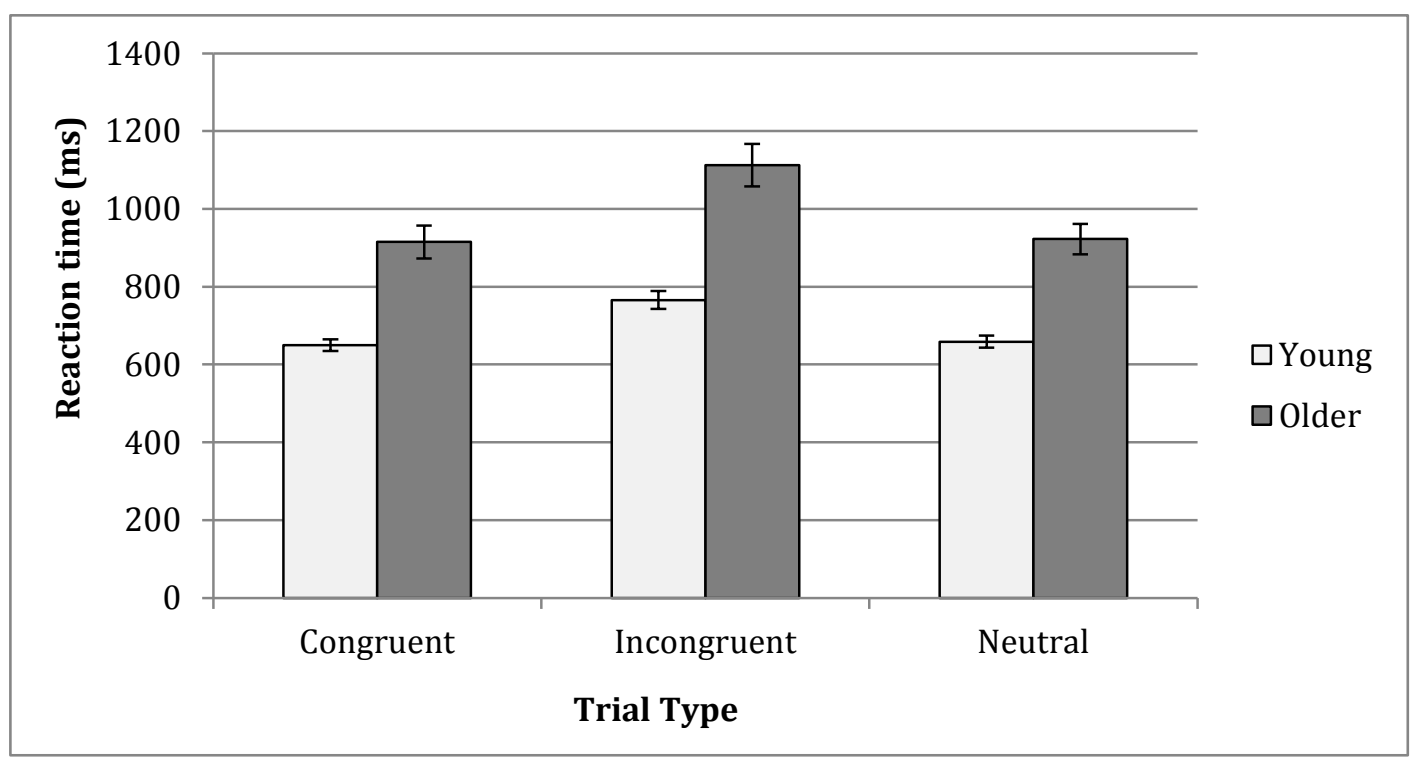

Figure 9. Mean RT in Stroop for congruent, incongruent, and neutral trials separately for young and older adults. Error bars represent the standard error.

Stroop interference (RT). Stroop RT interference scores were calculated by subtracting the RT of neutral from incongruent trials. An independent $t$ test on these scores showed greater interference for older $(M=190.53, S D=148.22)$ as compared to young adults $(M=106.99, S D=85.47), t(98)=-3.45, p=.001, d=.69$, supporting agerelated reductions in the restraint sub-function of inhibition. 


\section{AGE DIFFERENCES}

Table 7

Stroop: Accuracy, Reaction Time, and Interference Scores Separately for Young and Older Adults

\begin{tabular}{llll}
\hline Variable & $\begin{array}{l}\text { Young } \\
(n=50)\end{array}$ & $\begin{array}{l}\text { Older } \\
(n=50)\end{array}$ & $d$ \\
\hline Accuracy & & & \\
Congruent & $.96(.04)$ & $.98(.03)$ & .57 \\
Incongruent & $.90(.11)$ & $.95(.08)$ & .52 \\
Neutral & $.95(.04)$ & $.98(.03)$ & .85 \\
Interference & $.05(.12)$ & $.03(.06)$ & .21 \\
Reaction time & & & 1.18 \\
Congruent & $649.92(108.77)$ & $915.27(299.43)$ & 1.17 \\
Incongruent & $765.78(164.79)$ & $1113.01(386.22)$ & 1.25 \\
Neutral & $658.80(106.72)$ & $922.48(277.75)$ & 19 \\
Interference & $106.99(85.47)$ & $190.53(148.22)$ & .69 \\
\hline
\end{tabular}

Note. Standard deviations are in parentheses. Accuracy was denoted by the proportion of correct responses; RT was denoted in ms. Interference scores were indexed by the difference in performance between incongruent and neutral trials.

$d=$ Cohen's $d$ effect size calculation for between subjects .

\section{Summary}

To summarize, age differences (in either RT or accuracy) for Local-Global, Reading with Distraction, N-Back, Directed Forgetting, and Stroop were found ( $d s \geq .38$ ), including some trending effects ( $d s \geq .35$ ). The only task that did not show any reliable age differences at all was the Go - No Go task $(d \leq .11)$.

\section{Discussion}

In this study, age differences were explored in three sub-functions of inhibition using six different tasks. Overall, age differences were found in all three sub-functions of inhibition, with older adults showing poorer performance as compared to young adults; however, these patterns were not established consistently across all six tasks. The current 


\section{AGE DIFFERENCES}

findings add to previous work by Feyereisen and Charlot (2008) by demonstrating reliable age differences in access, deletion, and restraint - using additional tasks, such as Local-Global and N-Back.

\section{Access}

Local-Global. In the Local-Global task, age differences were established in both the overall interference effects and the preferential processing of local vs. global dimensions of a stimulus. Greater overall interference in older adults supports general age-related deficits in the ability to keep to-be-ignored irrelevant information outside one's focus of attention (i.e., access sub-function of inhibition). Furthermore, young adults showed greater interference scores during the local focus - with disruption coming from the global dimension, while older adults showed greater interference effects during the global focus - with disruption coming from the local dimension. These findings are in line with previous research on age differences in the Local-Global task (Navon, 1977; Slavin et al., 2002; Thomas et al., 2007), and suggest that older adults are particularly susceptible to distraction when it comes from the local dimension of a stimulus.

One explanation for the age-related shift away from global processing biases is neuroanatomical in that the brain regions associated with global processing deteriorate with age. A study by Delis, Roberston, and Efron (1986) investigated brain-lesion patients of varying ages (mean age less than 60 years) and concluded that global processing is more associated with the right posterior temporo-parietal regions, while local processing involves more left posterior temporo-parietal regions (see also Robertson \& Lamb, 1991). Therefore, it is possible that age-related changes to right posterior temporo-parietal brain regions (e.g., the right hemi-aging model; see Dolcos, Rice, \& 


\section{AGE DIFFERENCES}

Cabeza, 2002 for a brief review) or relative preservation of the left posterior temporoparietal regions that is underlying the age-related shift away from global processing and towards local features. It is believed that young adults process global information more readily, because overall visual and directed attention is right hemisphere dominant (Heilman \& Van Den Abell, 1980), and thus, assumedly, global features are prioritized in visual attention. Given that the proportion of right and left-handed participants was equivalent between the young and older adult samples, with disproportionately greater right-handedness in both samples, hand-dominance is an unlikely explanation of our age difference findings.

Slavin et al. (2002) also subscribe to the neuroanatomical explanation in their study in which they found a local precedence effect in older adults with probable or possible Alzheimer's disease, as well as age-matched controls. Interestingly, a subset of the participants with probable or possible Alzheimer's disease was not able to overtly identify the global dimension of the stimulus at all. This suggests that the right posterior temporo-parietal regions - associated with global processing - may undergo even greater changes in individuals suffering from probable or possible Alzheimer's disease than would be expected due to normal aging.

Future research should evaluate the neural correlates associated with the LocalGlobal task in young and healthy older adults to determine whether there are differences in the lateralization of brain activation during the task that coincide with the age-expected global precedence in young and local precedence (Slavin et al., 2002) or an absence of global precedence (Thomas et al., 2007) in older adults. Using a prospective study design, future research could also explore whether local precedence - as opposed to an 


\section{AGE DIFFERENCES}

absence of global precedence - in healthy older adults is indicative of underlying brain changes that predict the later development of pathology - e.g., probable or possible Alzheimer's disease.

Reading with Distraction. Older adults were trending to show greater differences in reading speed between high and low distracting conditions than young adults, with a small-to-medium effect size (see Cohen 1988, 1992 for effect size index). This marginal finding is largely in line with Connelly et al. (1991), where older adults showed significantly more reading speed disruption in high relative to low distracting passages than young adults. These marginal differences, however, are not in line with Feyereisen and Charlot (2008) who did not find age differences in reading speed difference scores. One possible reason for this discrepancy is a critical paradigm difference between studies. Specifically, in Feyereisen and Charlot (2008), the to-beignored words were italicized and the target words were not italicized. In the current study, the opposite was true. The paradigm used in Connelly et al. (1991) was followed where participants were instructed to read the italicized words out loud and ignore the words that were not italicized. Perhaps, older adults have an easier time keeping italicized words outside of their focus of attention, thereby reducing the associated distraction score and eliminating any age differences.

The proportion of distractor intrusions in the multiple-choice task was also examined. This subsequent analysis provide additional support to the trending age differences in passage reading speed and further suggest that older adults are letting more to-be-ignored information into their focus of attention and memory, subsequently incorporating it into their comprehension of the passage. This finding is, however, not in 


\section{AGE DIFFERENCES}

line with Connelly et al. (1991), where no age differences were found in distractor intrusions on the multiple-choice test. In the current study, overall accuracy performance is quite high on average (83\%); however, closer inspection of these data show that performance was greater than $80 \%$ for all except older adults in the high distracting condition, which was $74.5 \%$. This relatively lower accuracy performance in the multiplechoice task for high distracting passages means that older adults had more opportunities to make intrusion errors. This provides further evidence of the age-related deficits in access.

\section{Deletion}

N-Back. In the current study, evidence for reductions in overall accuracy in conditions with increasing demand on deletion was found. In particular, no evidence of age differences in the 0 and 1-Back conditions was found; however, age differences favouring young adults - began to emerge when participants were asked to match stimuli that appeared 2 and 3-Back in the sequence. The finding of greater age differences with higher N set sizes is in line with previous research (e.g., Dobbs \& Rule, 1989; Kirchner, 1958; Verhaeghen \& Basak, 2005) and offers support for age-related decline in the deletion sub-function of inhibition. These findings suggest that older adults are not effectively removing no longer relevant information from their working memory, so their storage space gets cluttered with both relevant and irrelevant stimuli making accurate responses to the current stimuli more challenging. In contrast, no age differences were found when no or minimal demands were placed on the deletion sub-function of inhibition (as in the 0-Back and 1-Back conditions). 


\section{AGE DIFFERENCES}

An alternative explanation for age differences in this task is an increase in task difficulty. In the current data, age differences in N-Back performance only began to emerge as the number of items that had to be held and manipulated in working memory increased - i.e., 2 and 3-Back. In general, as the task became more difficult, demands on cognitive resources also increased (Anderson \& Craik, 2000). Therefore, according to the reduced attentional resources theory of aging (Craik, 1983; Craik \& Byrd, 1982), older adults did not have sufficient resources available to perform under heightened levels of task complexity, which resulted in a reduction in accuracy (see also Craik \& Jennings, 2000). The inhibitory deficit hypothesis viewpoint (Hasher et al., 1999; Hasher et al., 2001; Hasher et al., 2007), however, suggests that older adults are unnecessarily using up valuable cognitive resources by processing information that is no longer task relevant (i.e., foil trials - for example, a stimulus that is three back in the sequence during a 2-Back block). Due to a limit in the number of foil trials included in this task, this specific hypothesis cannot be explored. Future research on age differences in the N-Back task would benefit from including a larger number of foil trials to enable an examination of age differences in accuracy to foils.

Directed Forgetting. An age effect was found in the time it takes to reject a TBF word as 'new'. In particular, older adults took a longer time than young adults to identify TBF words as 'new' as compared to correctly identifying NEW words as 'new'. This suggests that even though older adults were instructed to 'forget' and thus delete a given word from their working memory, they did not do so as effectively as young adults, resulting in a more muddled memory representation for the TBF words. Given this, it took older adults substantially longer to reject a TBF word as 'new' during the 


\section{AGE DIFFERENCES}

recognition task. These RT findings are in line with previous work on age-related deficits in the deletion sub-function of inhibition (Zacks et al., 1996).

The hit rate analysis in recognition revealed an overall directed forgetting effect with greater recognition for TBR relative to TBF words; however, this effect was shown to be equivalent in young and older adults. This finding is in line with previous research (Sego et al., 2006). Using recall, however, age differences in Directed Forgetting are more typically found (Sego et al., 2006; Zacks et al., 1996). Following this line of thinking, a recent meta-analysis by Titz and Verhaeghen (2010) suggest that smaller directed forgetting effects are found when recognition, instead of recall, is used to test memory. The age sensitivity of an outcome measure may also explain why evidence for age differences in directed forgetting was found when indexed by RT slowing to rejecting TBF words as 'new', but not recognition accuracy performance. Another explanation of our findings is that young adults are simply 'recalling to reject' the TBF words as 'new' more effectively than older adults. One way to assess this possibility is to include a questionnaire at the end of the study that asks participants whether they were making true ‘old/new’ judgments or not. Another option would be to implement a tagging procedure.

The use of a tagging procedure during the recognition phase of a Directed Forgetting paradigm (Thompson, Fawcett, \& Taylor, 2011) would minimize any potential issues regarding authenticity in responding and may also help to uncover more age sensitive differences in memory performance. Instead of using 'old' and 'new' response keys during recognition, tagging adds additional information by specifying three response options 'R' (if the participant recognizes the word as a TBR word), 'F' (if the participant recognizes the word as a TBF word), or ' $N$ ' (if the participant does not think they saw the 


\section{AGE DIFFERENCES}

word at all during encoding - i.e., is a 'new' word). Similar to the 'old' vs. 'new' response keys used in this study, efficient directed forgetting is identified by significantly greater ' $R$ ' responses to TBR words as compared to ' $F$ ' responses to TBF words (Thompson et al., 2011). However, in addition, using this procedure enables researchers to identify whether the false alarms (i.e., identifying a NEW word as 'old') were attributed to the TBR or TBF 'old' word and any associated age differences. In light of efficient directed forgetting, it is predicted that false alarms would more often be misattributed to ' $F$ ' rather than to ' $R$ ', because memory traces for TBR words are theoretically stronger than that of TBF words (Thompson et al., 2011). Therefore, it is expected that most of the false alarms committed by young adults in the current study would have been NEW words misattributed to TBF words. Using this tagging procedure would minimize response biasing on the part of the participant (e.g., responding 'new' to a TBF word because it was supposed to be forgotten) and also may help to uncover any hidden age differences in the false alarm rates. Therefore, this approach should be implemented in future Directed Forgetting studies that include a recognition component.

\section{Restraint}

Go-No Go. No age differences in false alarm rates were identified in the Go-No Go task. This is in line with previous findings (e.g., Allen et al., 1994; Falkenstein et al., 2002), suggesting that older adults do not decline in the ability to withhold an automatic motoric response. However, the results presented here must be interpreted with caution, due to the high miss rates observed in the data.

A closer examination of the experimental program identifies one possible explanation for the high miss rate and lack of age differences observed in the data. In 


\section{AGE DIFFERENCES}

particular, the stimulus was only on screen for 500 ms prior to the next trial commencing with a fixation cross. Responses were only recorded during this short 500 ms window, which may not have been a long enough time for participants to respond on 'Go' trials resulting in high miss rates. Given the nature of the false alarms (i.e., pressing the space bar on a 'No Go' trial), it is possible that false alarms were committed, but not recorded by the program. This program issue, therefore, may be masking any age differences in the Go-No Go task.

Previous studies that also failed to show age differences in the Go-No Go have potentially similar issues. For example, in Allen et al. (1994) and Falkenstein et al. (2002) stimulus presentation was only $100 \mathrm{~ms}$ and $300 \mathrm{~ms}$, respectively; however, in both studies an interstimulus interval was presented following the stimulus display during which time participants were able to make a response. Specifically, in Allen et al. (1994), participants were given up to $2000 \mathrm{~ms}$ to respond, and $1600 \mathrm{~ms}$ in Falkenstein et al., (2002). Interestingly, Falkenstein et al. encouraged participants to respond within a 400 ms time window post-stimulus onset using a feedback tone, if the time requirement was not met (2002). Future research, using older adult samples in particular, would benefit from implementing a Go-No Go task design with a longer stimulus display time or more time in which to respond - e.g., using an interstimulus interval.

Another possible explanation for the lack of age differences found was the simplicity of the task. In the Go-No Go task used here, only two types of stimuli were used 'Go' (e.g., '1') and 'No Go' (e.g., '9'). As a result of this simplicity, this task was a relatively pure measure of the restraint sub-function of inhibition; however, this may have contributed to the lack of age differences. Studies that have shown promising age 


\section{AGE DIFFERENCES}

differences in the Go-No Go task have done so using modified versions of the task. For example, in Nielson et al. (2002), participants were instructed to respond only to alternating targets - e.g., ' $\mathrm{X}$ ' and ' $\mathrm{Y}$ ' - imbedded in a series of individually presented letters (e.g., J-X-A-T-B-Y), and to withhold their response when the targets did not alternate (e.g., J-X-A-T-B-X). Although evidence of age differences were supported using this modified version of the Go-No Go, additional cognitive requirements embedded within this task, including dual-task processing, and the deletion of no longer relevant target letter information, make this version too process impure for our purposes.

Stroop. Age differences were established in the RT Stroop interference scores, with older adults showing significantly greater interference as compared to young adults. This finding is in line with the often-reported age-related increase in Stroop interference (Davidson et al., 2003; Dulaney and Rogers, 1994; Milham et al., 2002; West \& Alain, 2000), and suggests that older adults have more trouble withholding the automatic reading response during incongruent trials than young adults. These findings support agerelated declines in the restraint sub-function of inhibition.

Taken together, the current study shows, overall, there are age-related declines in access, deletion, and restraint; however, these effects were not found across all measures. In particular, age differences were established in RT or accuracy measures for both access tasks (i.e., Local-Global and Reading with Distraction), as well as both deletion tasks (i.e., N-Back and Directed Forgetting); however, only one restraint task (i.e., Stroop) showed age-related differences. No reliable effects of age were found in the Go - No Go task. These findings solidify the presence of declining inhibition in older adults and offer a rationale to pursue an investigation in the plasticity of all three sub- 


\section{AGE DIFFERENCES}

functions among older adults. The final chapter in this dissertation will investigate this avenue of research. 


\section{PLASTICITY OF THREE SUB-FUNCTIONS OF INHIBITION}

Chapter 3. Plasticity of Three Sub-functions of Inhibition in Older Adults:

\section{Retest Practice and Transfer Effects}

Age-related deficits in all three sub-functions of inhibition have been demonstrated in previous literature (Connelly et al., 1991; Davidson et al., 2003; Dobbs \& Rule, 1989; Dulaney \& Rogers, 1994; Kirchner, 1958; McAvinue et al., 2012; Milham et al., 2002; Slavin et al., 2002; Thomas et al., 2007; Verhaeghen \& Basak, 2005; West \& Alain, 2000; Zacks et al., 1996). Such findings were replicated in the study reported in Chapter 2; however, to my knowledge, no previous work has been done to examine the plasticity of all three sub-functions of inhibition - within the same group of older adults using a multi-task approach. The current study will fill this gap using three critical training tasks: Local-Global (access), N-Back (deletion), and Go - No Go (restraint). Furthermore, transfer effects will also be examined. The tasks used to train the three subfunctions of inhibition were chosen for several reasons: (a) process purity with respect to the targeted ability, (b) ease of modifiability across six sessions, and (c) ease of adaptability into a near-near transfer task.

According to the inhibitory deficit hypothesis of aging, age-related declines in inhibition result in other cognitive deficits, such as speed of processing and memory. However, our previous work did not find any transfer effects following inhibition training to other cognitive domains using a single-task approach (Wilkinson \& Yang, 2012). Given that inhibition is often considered to be a multi-function cognitive ability (English \& English, 1958; Friedman \& Miyake, 2004; Dempster, 1993; Hasher et al., 1999; Hasher et al., 2007; Kipp Harnishfeger, 1995; Nigg, 2000; Wilson \& Kipp, 1998), one 


\section{PLASTICITY OF THREE SUB-FUNCTIONS OF INHIBITION}

possible explanation for this lack of transfer is that a single task approach to training inhibition does not activate a wide enough set of brain regions to elicit transfer effects.

To assess the benefits of training all three sub-functions of inhibition to other cognitive abilities the current study will take a novel approach and evaluate three levels of transfer: (a) near-near transfer is assessed using the exact same task as used during the training, but with varying items; (b) near transfer is assessed with a task that is different from that used in training, but thought to tap the same ability. Here, Reading with Distraction will be used as the outcome measure for access, Directed Forgetting for deletion, and Stroop for restraint; and (c) far transfer is when the transfer and training tasks do not overlap at all in terms of task structure or tapped cognitive domain. Here, far transfer will be measured in working memory (using the Corsi Block task), episodic memory (Word List Recall), reasoning (Letter Series), and speed of processing (Digit Symbol).

To summarize, the current study will address two research questions: (a) do older adults demonstrate plasticity in all three sub-functions of inhibition: access, deletion and restraint?; and (b) does training three sub-functions of inhibition in older adults elicit near-near, near and/or far transfer effects? Based on literature, it is hypothesized that all three sub-functions of inhibition will show practice-induced improvement, and the training is expected to elicit near-near transfer effects. Transfer to near and far tasks, although theoretically supported (inhibitory deficit hypothesis; see Hasher et al., 1999; Hasher et al., 2007), are more exploratory in nature, so no firm hypotheses are specified. 


\section{PLASTICITY OF THREE SUB-FUNCTIONS OF INHIBITION}

\section{Method}

\section{Participants}

Forty-eight older adults (14 males and 34 females, age range $=60-88$ years; $M=70.25, S D=7.79$ ) were recruited to participate in this study. Pretest data from all 48 participants was included as part of the older adult sample for the age differences study (see Chapter 2 of this dissertation). Twenty-four were assigned to the training group (17 females, age range $=60-88$ years; $M=68.96, S D=8.13$ ) and 24 to the control group (17 females, age range $=61-87$ years; $M=71.54, S D=7.37$ ). All participants provided informed consent according to the REB at Ryerson University (see Appendices $\mathrm{I}$ and $\mathrm{J}$ for the consent agreement provided to the training and control groups, respectively). Three participants (2 training, 1 control) presented with colour blindness, as measured with the Dvorine Pseudo-Isochromatic Plates (Dvorine Pseudo-Isochromatic Plates, 1953). The Stroop analyses were conducted with and without these three subjects and the results stayed the same; so, their data were included in all of the final analyses. All participants had reasonable to normal near vision, after correction if applicable (range 20/20 - 20/50), as measured with the Rosenbaum near acuity pocket screener (Rosenbaum Pocket Vision Screener, 1986). No participants showed dementia related cognitive impairment as screened with the Short Blessed Test (SBT; Katzman et al., 1983), using a cut-off score of 6 (range 0 - 5). No participants reported severe anxiety, as reflected in the scores $(<26)$ on the Beck Anxiety Inventory (BAI; Beck et al., 1988). All participants were debriefed at the end of the study and compensated \$10 per hour for their time (see Appendices $\mathrm{K}$ and L for the debriefing form provided to the training and control groups, respectively). 


\section{PLASTICITY OF THREE SUB-FUNCTIONS OF INHIBITION}

The training and control group did not differ in any baseline cognitive performance or demographic variables (all $p s \geq .09$; see Table 8).

\section{Design and Procedure}

All participants completed a 2.5-hour pretest session, followed by two weeks of experimental manipulation, and then a 2-hour posttest session. For those in the training group, the 2-week interval consisted of six 30-minute in lab retest practice sessions (three times per week for two consecutive weeks). For those in the control group, the 2-week interval consisted of 'no contact' (i.e., they did not receive any training).

\section{Materials and Stimuli}

A 17-inch monitor PC computer was used for all of the computerized tasks. All participants were comfortably seated in a well-lit testing room at a viewing distance of approximately $60 \mathrm{~cm}$. 


\section{PLASTICITY OF THREE SUB-FUNCTIONS OF INHIBITION}

Table 8

Demographic Characteristics and Baseline Cognitive Performance Assessed at Pretest Separately for Training and No Training Control

\begin{tabular}{lllll}
\hline Characteristic & $\begin{array}{l}\text { Training } \\
(n=24)\end{array}$ & $\begin{array}{c}\text { No Training Control } \\
(n=24)\end{array}$ & $p$ & $d$ \\
\hline Age (yrs) & $68.96(8.13)$ & $71.54(7.37)$ & .26 & .33 \\
Education (yrs) & $15.83(3.56)$ & $15.71(2.97)$ & .90 & .04 \\
Health & $8.43(1.34)$ & $8.58(1.25)$ & .70 & .12 \\
Visual acuity & $26.25(6.47)$ & $27.29(5.89)$ & .56 & .17 \\
Letter Series & $10.50(4.28)$ & $10.38(4.17)$ & .92 & .03 \\
Digit Symbol & $61.46(15.37)$ & $62.25(14.79)$ & .86 & .05 \\
Recall - Correct & $.40(.35)$ & $.45(.31)$ & .63 & .15 \\
Recall - Correct Right Sequence & $.14(.21)$ & $.05(.11)$ & .09 & .54 \\
Recall - Correct Wrong Sequence & $.07(.09)$ & $.08(.09)$ & .68 & .11 \\
Recall - Correct Overall & $.61(.32)$ & $.58(.26)$ & .76 & .10 \\
Recall - Wrong Word & $.04(.05)$ & $.06(.09)$ & .34 & .27 \\
Beck’s Anxiety Inventory & $4.63(4.03)$ & $6.17(6.78)$ & .34 & .28 \\
Shipley Vocabulary Test & $36.63(1.97)$ & $35.96(2.79)$ & .34 & .28 \\
CES-D & $8.83(6.16)$ & $9.92(8.52)$ & .62 & .15 \\
Short Blessed Test & $.75(1.29)$ & $.71(1.52)$ & .92 & .03 \\
\hline
\end{tabular}

Note. Standard deviations are in parentheses. CES-D = Centre for Epidemiological Studies of Depression Scale. Education was indexed by the average number of years of formal education; Health was indexed by a self-reported score out of 10; Visual acuity was indexed by the near visual acuity score from the Rosenbaum Visual Acuity Pocket Screener (score 20/--); Letter Series, Digit Symbol, and Shipley were scored by the average number of correct solutions; Recall denotes the proportion of words recalled in each of the pre-specified categories during the Word List Recall Test; Average scores were displayed for Beck’s Anxiety Inventory, CES-D, and Short Blessed Test.

${ }^{*} p<.05 ; d=$ Cohen's $d$ effect size calculation for between subjects.

Practice materials and stimuli. At each of the six training sessions, participants completed three tasks: Local-Global, N-Back, and Go - No Go. The task order was counterbalanced across participants. Since the general task structure has been described 


\section{PLASTICITY OF THREE SUB-FUNCTIONS OF INHIBITION}

previously (see General Introduction and Methods section of Chapter 2), only the most pertinent and unique details to this training study will be described below.

Local-Global. The Local-Global task was modeled after Navon (1977) and Thomas et al. (2007). In this task, participants were presented with images that consisted of large letters (global dimension; e.g., A, D, H, L) that were composed of small letters (local dimension; e.g., A, D, H, L). Two out of the four letters were considered targets (e.g., 'A' and 'D') and two as control stimuli (e.g., 'H' and 'L'). Prior to each block, participants were instructed to attend to either the large letters or the small letters and to respond with one of two target letter options (e.g., 'A' or 'D'). Specifically, the task involved three types of trials: congruent, incongruent and neutral (see page 10 for sample stimuli). As mentioned previously, the dependent variables of interest were overall RT, as well as interference scores calculated separately for the local and global dimension focus (i.e., incongruent RT - congruent RT; Kotchoubey et al., 1997; Navon, 1977).

Instructions to focus on the local or global dimension of the stimuli were presented in a blocked design - counterbalanced across participants, but kept consistent within-participants across sessions. For additional information on task procedure, please see the Method section in Chapter 2. The letter stimuli were selected from the whole alphabet - except ' $M$ ' and ' $W$ '. Four letters were randomly selected for each session, with two serving as the target stimuli and two serving as control. The target and control stimuli - within a session - were counterbalanced across participants.

N-Back. The N-Back task was modified from Braver et al. (1997). During the six practice sessions, three different N-back conditions were included: 1-Back, 2-Back and 3-Back. The conditions were presented in a blocked design, in the same order (ascending 


\section{PLASTICITY OF THREE SUB-FUNCTIONS OF INHIBITION}

from 1-Back to 3-Back) for all sessions. Stimuli were selected from 20 consonant letters presented in upper and lower case (excluding vowels and ' $\mathrm{Y}$ ') for the practice sessions.

Participants first completed three practice blocks, one for each condition and 10 trials for each block, followed by three experimental blocks, one for each condition and 45 trials for each experimental block. Each experimental block contained nine target trials and 36 non-target trials presented in pseudorandom order. Six of the non-target trials were foils - three for each of the non-tested alternative experimental condition. As a reminder, non-target foils were trials that would have been considered a 'TARGET' trial, if presented in a different condition block. For example, if the series of letters: B-a-C-b was presented in the 2-back block, the second 'b' would be considered a 3-Back foil trial, because it would have been a 'TARGET' trial had it been presented in the 3-Back block. As mentioned previously, overall accuracy for each condition (1-Back, 2-Back and 3-Back) was considered as the dependent variable of interest. Specifically, efficient deletion would be indicated by higher accuracy scores - especially during the more difficult 2-Back and 3-Back conditions.

Go-No Go. The Go-No Go task was modeled after Donders (1868/1969). In this task, participants were shown letter stimuli - one at a time - on the computer screen. They were instructed to press the spacebar when a pre-specified letter (e.g., 'O') appeared on the screen - i.e., 'Go' trial - and to withhold their response (i.e., do not press the space bar), when a different, pre-specified letter (e.g., ' $X$ ') appeared on the screen - i.e., 'No Go’ trial. Letter stimuli were selected from B, D, E, F, K, M, P, R, U, V, W, and X. Two letters were randomly selected for each session, and the assignment to 'Go' or ‘No Go’ conditions was counterbalanced across participants. As mentioned previously, 


\section{PLASTICITY OF THREE SUB-FUNCTIONS OF INHIBITION}

the false alarm rate (i.e., the number of false alarms committed divided by the total number of 'No-Go' trials [50]) was used to measure the restraint function of inhibition. In particular, smaller false alarm rate represent greater restraint.

Pretest and posttest materials and stimuli. To assess possible transfer effects, a battery of cognitive tasks was administered at pretest and posttest sessions (see Table 9 for a list). The pretest and posttest tasks were selected to assess three levels of transfer effects: near-near, near, and far. Different parallel versions of the all tasks (except the BAI, Shipley, and CES-D) were administered at pretest and posttest sessions to reduce repetition, and minimize item-specific effect. 


\section{PLASTICITY OF THREE SUB-FUNCTIONS OF INHIBITION}

Table 9

List of Tasks Administered at Pretest, Training, and Posttest Sessions

\begin{tabular}{lccc}
\hline Task & Pretest & Training & Posttest \\
\hline Local-Global & $\checkmark$ & $\checkmark$ & $\checkmark$ \\
N-Back & $\checkmark$ & $\checkmark$ & $\checkmark$ \\
Go-No Go & $\checkmark$ & \\
Reading with Distraction & $\checkmark$ & $\checkmark$ \\
Directed Forgetting & $\checkmark$ & $\checkmark$ \\
Stroop & $\checkmark$ & $\checkmark$ \\
Corsi-Block Visuospatial Working Memory & $\checkmark$ & $\checkmark$ \\
Word List Recall & $\checkmark$ & $\checkmark$ \\
Letter Series & $\checkmark$ & $\checkmark$ \\
Digit Symbol & $\checkmark$ & \\
Beck’s Anxiety Inventory & $\checkmark$ & \\
Shipley Vocabulary Test & $\checkmark$ & \\
Centre for Epidemiological Studies of Depression Scale & $\checkmark$ & \\
Short Blessed Test & $\checkmark$ & \\
\hline
\end{tabular}

Near-near transfer. The Local-Global, N-Back, and Go-No Go tasks with varying items (i.e., digit instead of letter) were administered to evaluate near-near transfer effects in each of the three sub-function of inhibition: access, deletion, and restraint, respectively.

Local-Global. For the Local-Global task as a near transfer outcome measure, the number stimuli were $1,2,3$, and 4 at pretest and $5,6,7$, and 8 at posttest. The target numbers at pretest were assigned as 2 and 3 (i.e., 1 and 4 were control stimuli) or 1 and 4 


\section{PLASTICITY OF THREE SUB-FUNCTIONS OF INHIBITION}

(i.e., 2 and 3 were control stimuli). At posttest, the target stimuli were assigned as 5 and 6 (i.e., 7 and 8 were control stimuli) or 7 and 8 (i.e., 5 and 6 were control stimuli). The target and control number assignment was counterbalanced across participants and response keys ('z’ and ‘/').

$\mathrm{N}$-Back. For the N-back task as a near transfer outcome measure, the numbers included ranged from 1 to 9. The 1-Back, 2-Back and 3-Back blocks were presented in the exact same format as during the training sessions; however, a 0-Back block was included as the initial block. In the 0-back condition, participants were instructed to indicate whether a pre-specified number (e.g., '5') appeared at all by pressing the key labeled 'TARGET,' if it did, and 'NON-TARGET', if any other number was presented. Two trial lists were created - one was administered at pretest and the other at posttest. Key assignment ('z' or '/' for 'TARGET' or 'NON-TARGET') was counterbalanced across participants, but kept consistent within-participants across sessions

Go-No Go. For the digit Go - No Go task, number pairs of 1 and 9 or 4 and 8 were used as stimuli for 'Go' and 'No Go' responses, respectively. Within each pair, the number assignment to the 'Go' or 'No Go' condition was counterbalanced across participants. In addition, the number pairs (1 and 9 or 4 and 8) were counterbalanced across pretest and posttest sessions.

Near transfer. The tasks used to assess near transfer effects in the three subfunctions of inhibition: access, deletion, and restraint were Reading with Distraction, Directed Forgetting, and the Stroop task, respectively.

Reading with Distraction. The Reading with Distraction task was modified from Connelly et al. (1991). In this task, participants were asked to read an individually 


\section{PLASTICITY OF THREE SUB-FUNCTIONS OF INHIBITION}

presented short passage out loud. There were eight passages in total (four high distracting and four low distracting). Four different passages (two high distracting and two low distracting) were presented at pretest and four at posttest - in varying order, counterbalanced across participants. The condition presentation alternated between high and low distraction (i.e., H-L-H-L or L-H-L-H) and was counterbalanced across participants, but kept consistent within-participants across sessions. For example, if a participant was presented with an order of H-L-H-L at pretest, they were presented with the same at posttest (but with different passages). The dependent variable of interest was the difference in reading speed between high and low distracting passages, and accuracy (particularly distractor intrusions for the high distracting condition) on the multiplechoice questions.

Directed Forgetting. The Directed Forgetting task was modeled after Sego et al., (2006; also see MacLeod, 1998, 1999). Two versions of this task were created - with varying words. Administration of the different versions was counterbalanced across participants and session. For additional information on stimuli construction and task procedure, please see the Method section in Chapter 2.

As mentioned previously, there were two dependent variables of interest: (a) RT for responding 'new' to TBF and NEW words, and (b) hit rate for TBR and TBF words.

Stroop. The Stroop task was modeled after the one used in Wilkinson and Yang (2012) and adapted from the classical paradigm developed by Stroop (1935). Briefly, in this task, participants were instructed to respond to the ink colour of the stimuli by pressing the correspondingly assigned colour-coded response keys on the keyboard (i.e., 'z', 'x', '.', '/’). Two participants in the training group used the correspondingly labeled 


\section{PLASTICITY OF THREE SUB-FUNCTIONS OF INHIBITION}

number keypad to make their responses (i.e., '1', '2', '4', ‘5'). All Stroop analyses were conducted with and without these participants, and the results stayed the same; so, their data were included in the final analysis. The two versions of Stroop task were created and presentation was counterbalanced across participants and session. For additional information on stimuli construction and task procedure, please see Method section of Chapter 2. As mentioned previously, Stroop interference scores - calculated as the difference in RT and accuracy between incongruent and neutral trials during the experimental block - was used as the dependent variable.

Far transfer. The tasks used to assess far transfer effects included the Corsi Block, Word List Recall Test, Letter Series and Digit Symbol.

Corsi Block. A computerized version of the Corsi Block visuospatial working memory span task (a modified mouse version from Rowe, Hasher, and Turcotte, 2009; see also Corsi, 1972) was used to assess working memory. In this task, participants were presented with a display of nine grey squares on a white background for $1200 \mathrm{~ms}$. Next, some of the squares would turn black - for 1000 ms each - one at a time in a sequence. Participants were asked to remember and then reproduce the sequence of squares that turned black by clicking the mouse cursor on the squares. Participants completed six practice trials: three 2-span and three 3-span trials. Next, participants completed the experimental trials, where the length of the sequence varied between four to seven squares (three trials for each sequence length). Span length was always presented in an ascending order (i.e., 4 to 7). The proportion of accurate responses within a trial was calculated and then averaged across each span length to provide a measure of working memory as modulated by set size. In addition, following Rowe et al. (2009), overall 


\section{PLASTICITY OF THREE SUB-FUNCTIONS OF INHIBITION}

accuracy performance (i.e., the proportion of trials that were correctly recalled in the right sequence) was also examined. Two trial lists were created and counterbalanced across participants and session.

Word List Recall. To assess episodic memory, a Word List Recall test was administered. In this test, two lists of 13 words each were selected and matched on critical psycholinguistic characteristics, including concreteness, frequency, length, number of syllables, familiarity, imaginability, valence, arousal, and dominance - using Warriner, Kuperman, and Brysbaert's (2013) extension of the ANEW database (Bradley \& Lang, 1999). Two parallel word lists were borrowed from Yang, Reed, and Wilkinson (2009b) and counterbalanced across participants and session.

Participants were given three minutes to study the word list, including the sequence, for a later memory test. Participants then completed two filler tasks for eight minutes: Letter Series (six minutes) and Digit Symbol (two minutes) - described in detail below. Following the filler tasks, participants were asked to write down as many words as they could remember in the same sequence as they were presented in the study list. There was no enforced time limit for the free recall.

Five dependent variables of interest were specified: correct, correct in right sequence, correct in wrong sequence, correct overall, and wrong word. To illustrate the meaning of the five dependent variables of interest, consider that the following list of words was presented during encoding: magazine, elbow, cigar, and author. The focus in this explanation will be on the word elbow. For a given word to be considered 'correct', the exact word from the encoding list would have to be recalled in the identical numbered sequence slot as it was originally presented (e.g., magazine, elbow, cigar, author). For a 


\section{PLASTICITY OF THREE SUB-FUNCTIONS OF INHIBITION}

word to be considered 'correct, right sequence', the exact word would have to be recalled, but in the wrong numbered sequence slot, although replicating the serial position at encoding - i.e., listed directly above or below the same word as presented during encoding (e.g., iron, skin, elbow, cigar). For a word to be counted as 'correct, wrong sequence', the exact word would have to be recalled but in the wrong numbered sequence slot, and not replicating the serial position at encoding (e.g., skin, brain, elbow, author). Correct overall is the number of words correctly recalled, regardless of sequence accuracy. A 'wrong word' was one that did not appear in the encoding list at all (e.g., grill, banana, vacuum, desk). The plural version of a word was accepted as correct as were words recalled in the wrong tense (e.g., 'sprayed' instead of 'spray'). However, a person noun that was incorrectly recalled as an object noun (e.g., 'art' instead of 'artist') or vice versa (e.g., 'trucker' instead of 'truck') was scored as incorrect. Last, if a word was recalled twice, only the first response was scored.

Letter Series. The Letter Series was used as a measure of inductive reasoning (Blieszner, Willis, \& Baltes, 1981). In this test, participants viewed a series of letters and were required to decipher the pattern/rule in each series. Based on this pattern/rule, participants were required to pick one letter, from a possible five, that would best continue the pattern (e.g., for the series ' $\mathrm{z} f \mathrm{y}$ e $\mathrm{x} \mathrm{d} \_$_, the correct response would be 'w'). Participants were given six minutes to complete up to 20 letter series problems. Two parallel versions of the Letter Series were borrowed from Yang et al. (2009a) and counterbalanced across pretest and posttest session. The number of correct responses was used as a measure of reasoning ability. 


\section{PLASTICITY OF THREE SUB-FUNCTIONS OF INHIBITION}

Digit Symbol. The task used to assess speed of processing was the Digit Symbol (Wechsler, 1981). On a single piece of paper, participants were presented with 140 digits in squares (including 7 practice trials), each with a corresponding empty square underneath. Participants were required to draw in the corresponding symbol based on a conversion code presented at the top of the page. The experimenter demonstrated the first practice trial, and then participants were asked to complete the remaining six practice trials on their own. Following this, participants were given two minutes to complete as many items as possible, one at a time, without skipping any. Two parallel versions of the Digit Symbol were borrowed from Yang et al. (2009a) and counterbalanced across pretest and posttest session. The number of correct responses was used as a measure of processing speed.

\section{Results}

All analyses were conducted using the IBM SPSS Statistics 20.0. First, the data processing and cleaning procedure will be described for the Local-Global, N-Back, and Go-No Go tasks across the practice and pretest/posttest data combined. Next, the retest practice effects will be examined. Finally, the transfer effect data will be evaluated in the following order: near-near transfer (Local-Global, N-Back and Go-No Go), near transfer (Reading with Distraction, Directed Forgetting, and Stroop), and far transfer (Corsi Block, Word List Recall Test, Letter Series and Digit Symbol). For each of the near and far transfer tasks, the data processing and cleaning procedures will first be described, and then report the transfer effect findings. All of the analyses reported below (i.e., practice and transfer effects) involving multiple comparisons were adjusted with Bonferroni Correction. 


\section{PLASTICITY OF THREE SUB-FUNCTIONS OF INHIBITION}

\section{Data Processing (Local-Global, N-Back, and Go-No Go)}

The Local-Global, N-Back and Go-No Go data from the practice, and the pretest/posttest sessions were processed together for simplicity.

Local-Global. Only RTs for correct trials were used in the analyses $(1.44 \%$ of trials were removed). Furthermore, RTs that were more than 2.5 SDs away from the cell mean and/or more than 5000 ms were removed as outliers (3.37\%). Last, any RTs less than 200 ms (0.02\%, signaling premature responses) were deleted. Mean RTs were then calculated separately for each trial type (congruent, incongruent and neutral), in each dimension (local and global), at each session (pretest, posttest, six practice sessions).

Missing trials. At the practice sessions, data from three experimental blocks (two local and one global) were not recorded properly due to the program crashing. Practice session 3 and 5 were affected for three different participants. These missing data points were predicted using separate linear regression models for each participant. For example, one participant was missing RT data for congruent trials in the local dimension at session 3. Using session (i.e., $1-6$ ) as the predictor, and the RT data from the completed five sessions as the dependent variable, a linear regression equation was generated: $-4.51 x+507.88$. The missing session number was then inserted into the equation $-4.51(3)+507.88$ to yield the missing data point - i.e., 494.35. In addition, one of the response keys stopped working halfway through the global block for one participant during session 4 . The affected trials were removed (36.1\% of the global condition) and the mean RTs for this block were calculated based on the remaining trials.

N-Back. After removing all of the 'no response detected' trials from the data set (2.31\%), the proportion of accurate responses was calculated separately for each 


\section{PLASTICITY OF THREE SUB-FUNCTIONS OF INHIBITION}

condition (0-Back, 1-Back, 2-Back, and 3-Back) at each session (pretest, posttest, six practice sessions). RT scores were not included in the analysis of the N-Back data for two key reasons. First, the design of the study required that participants wait for the fixation cross to appear prior to making a response, which resulted in some extremely fast RTs perhaps due to hitting the key repeatedly prior to the onset of the fixation cross. Second, participants were limited to a 2000 ms time window for responding. Given these reasons, the RT data may have been artificially reduced, so were not further analyzed.

Missing trials. One participant chose to stop responding during the 3-Back block of the pretest session; so, only those trials with a response were used to calculate this particular mean. In addition, the response key stopped working during session 4 (2-Back and 3-Back) and posttest (0-Back) for the same participant. The missing cells for the session 4 data were imputed using the regression model with session as the predictor. Unfortunately, this method could not be implemented to predict the missing posttest data, because data from only one of two sessions (i.e., pretest and posttest) was available. Therefore, this remained a missing data point. Last, the E-Prime program crashed three times (inconsistently), which resulted in fewer generated trials. The affected sessions and blocks, from different participants, included pretest (2-Back), pretest (3-Back) and session 3 (3-Back). In these cases, only the program-generated trials (prior to the program crashing) were used to calculate the associated means. As a result of the program crashing during the 2-Back block at pretest (mentioned above), this particular participant did not have any 3-Back trials for this session. Again, these pretest data points could not be imputed using a regression model, so they were left as missing data points. In sum, 


\section{PLASTICITY OF THREE SUB-FUNCTIONS OF INHIBITION}

any missing data at the pretest and posttest sessions were left as missing data points, while those at the practice sessions were imputed with a linear regression model.

Go-No Go. The false alarm rate was calculated by dividing the number of times the spacebar was hit on a 'No Go' trial by the total number of 'No Go' trials for each session separately (i.e., pretest, posttest, six practice sessions).

\section{Practice Effects}

To best capture the nature and trajectory of the training benefits, all session effect analyses specified the linear (suggesting incremental improvement) and quadratic contrasts (reflecting saturation or ' $U$ ' shaped effects).

\section{Local-Global.}

Overall RT. Overall RT data were submitted to a 2 (dimension: local vs. global) $\times$ 3 (trial type: congruent, incongruent vs. neutral) $\times 6$ (session) repeated measures ANOVA. The overall RT analysis revealed a significant session effect in both linear, $F(1,23)=12.01, p=.002, \eta^{2}=.34$, and quadratic contrasts, $F(1,23)=9.26, p=.01$, $\eta^{2}=.29$. There was also a significant effect of trial type, $F(2,46)=51.97, p<.001$, $\eta^{2}=.69$. Post hoc paired $t$ tests revealed that the RTs from all three trial types were significantly different from each other: congruent $(M=575.04, S D=91.59)$, incongruent $(M=627.88, S D=117.09)$, and neutral $(M=617.73, S D=114.99)$, all $p s \leq .007$. In addition, the main effect of dimension was approaching, but failed to reach significance, $F(1,23)=3.96, p=.06, \eta^{2}=.15$. In particular, there was a trend towards faster responses to the global dimension $(M=593.10, S D=98.71)$ as compared to local dimension $(M=620.67, S D=125.00)$. Given the significant session $\times$ dimension interaction (described below), it is possible that the trending global precedence effect found in our 


\section{PLASTICITY OF THREE SUB-FUNCTIONS OF INHIBITION}

older adult sample is driven by greater improvements in the global dimension across sessions. To explore this possibility, the difference in overall RT performance between the local and global dimensions at session 1 (before any practice related improvements had taken place) was examined using a paired sample $t$ test. Results revealed no significant difference between the overall RT of the local and global dimensions at session $1, t(23)=-1.35, p=.19$, suggesting an absence of global precedence effect in this older adult sample.

The main effects were qualified by four significant interactions. First, there was a significant session $\times$ trial type interaction, $F(10,230)=2.96, p=.002, \eta^{2}=.11$. In order to tease apart this interaction, repeated measure ANOVAs (session) were run for each trial type separately - collapsed across condition. All three trial types showed significant linear and quadratic session effects (all $p s \leq .02$ ). To explore which trial type showed the larger session effect slopes from the linear regression model were examined and curve fit analysis, respectively, to provide a measure of improvement rate. The linear slope was -8.39 for the congruent trials, -14.88 for the incongruent trials, and -14.54 for the neutral trials, suggesting a steeper linear improvement in incongruent and neutral relative to congruent trials. Given that the session effect was significant in the quadratic contrast for all three trial types as well, the slopes generated for the two segments of this curved data pattern (using curve fit analyses) were examined. Given that the purpose was to evaluate which trial type underwent greater practice induced improvement, the focus will be on the first segment of the curve - i.e., the initial part of the curve that slopes downwards (until about session 4). The associated slope was -53.60 for congruent trials, -71.00 for 


\section{PLASTICITY OF THREE SUB-FUNCTIONS OF INHIBITION}

incongruent trials, and -57.71 for neutral trials. This suggests that the incongruent trials showed larger session effects during earlier sessions (i.e., 1 to 4).

There was also a significant session $\times$ dimension interaction in both linear, $F(1,23)=15.19, p=.001, \eta^{2}=.40$ and quadratic contrasts, $F(1,23)=4.68, p=.04$, $\eta^{2}=.17$. To further explore this interaction, repeated measure ANOVAs (session) were run for each dimension separately - collapsed across trial type. For the local dimension, only the linear session effect was significant, $F(1,23)=4.90, p=.04, \eta^{2}=.18$. For the global dimension, the session effect was significant in both linear and quadratic contrasts, ps $\leq .001$. The linear slope was -6.76 for the local dimension and -18.45 for the global dimension, suggesting greater incremental improvements in the global as compared to the local dimension.

There was also a significant trial type $\times$ dimension interaction, $F(2,46)=4.47$, $p=.02, \eta^{2}=.16$. To evaluate this interaction, the three trial types were compared separately for each dimension using paired $t$ tests. For the local dimension, congruent trials $(M=585.07, S D=105.69)$ were responded to significant faster than incongruent $(M=638.51, S D=134.79)$ and neutral trials $(M=638.42, S D=137.16), p s<.001$. The latter two did not differ significantly, $p=.98$. For the global dimension, responses to congruent trials $(M=565.01, S D=84.48)$ were significantly faster than to neutral trials $(M=597.04, S D=103.16)$, which in turn were faster than those to incongruent trials $(M=617.25, S D=111.33)$, all $p s<.001$.

Last, the higher-order three-way session $\times$ trial type $\times$ dimension interaction was significant, $F(10,230)=3.69, p<.001, \eta^{2}=.14$. To further explore this interaction, repeated measure ANOVAs (session) were run for each trial type separately for the local 


\section{PLASTICITY OF THREE SUB-FUNCTIONS OF INHIBITION}

and global dimension. For the local condition, the session effect was significant in linear contrast for incongruent and neutral trials ( $p$ s $\leq .02$ ), but not for congruent trials, $p=.71$. The quadratic contrast, however, was significant for congruent trials only, $p=.03$ (other $p s>.50$ ), suggesting that congruent trials followed a ' $U$ ' shaped pattern (apparent most prominently at session 6), while incongruent and neutral trials showed continuous incremental improvement across sessions. The linear regression slope showed a surprisingly steeper slope in neutral (-11.39) relative to incongruent trials (-7.75). For the quadratic curve analysis (specifying the slope of the initial portion of the curve), the local congruent trials had a slope of -42.86 (see Figure 10).

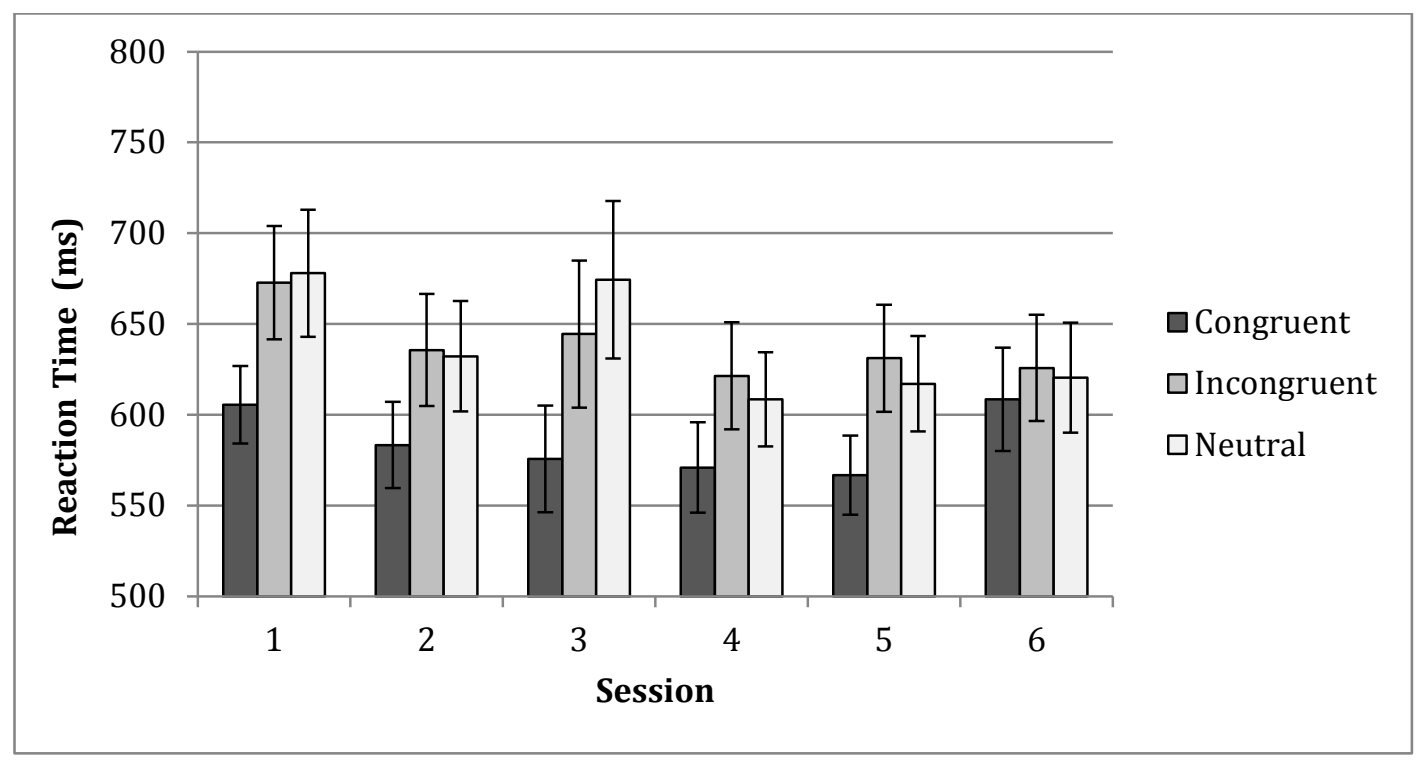

Figure 10. Mean RT in Local-Global with local dimension focus for congruent, incongruent, and neutral trials across six practice sessions. Error bars represent the standard error.

For the global dimension, the session effect was significant in both linear and quadratic contrasts for all three trial types (all $p s \leq .01$ ). In particular, the linear slope was steeper for incongruent trials (-22.00) relative to congruent (-15.64) and neutral trials (-17.70). For the quadratic curve analysis (specifying the slope of the initial portion of the 


\section{PLASTICITY OF THREE SUB-FUNCTIONS OF INHIBITION}

curve), the congruent slope was -64.34 , incongruent was -113.95 , and neutral was -90.14 (see Figure 11). The slope values, as well as visual inspection, suggest a steeper improvement across practice sessions in the global dimension overall. Furthermore, the breakdown of the 3-way interaction suggests that the steepest improvement is found in the incongruent trials during global dimension focus (where local information is interfering with performance). These observations are in line with the diminishing global precedence effect typically found with increasing age and support the idea that the ability to keep to-be-ignored local information outside one's focus of attention can be improved with practice. This possibility will be further explored with interference scores (incongruent RT - congruent RT) calculated separately during local and global dimension focus.

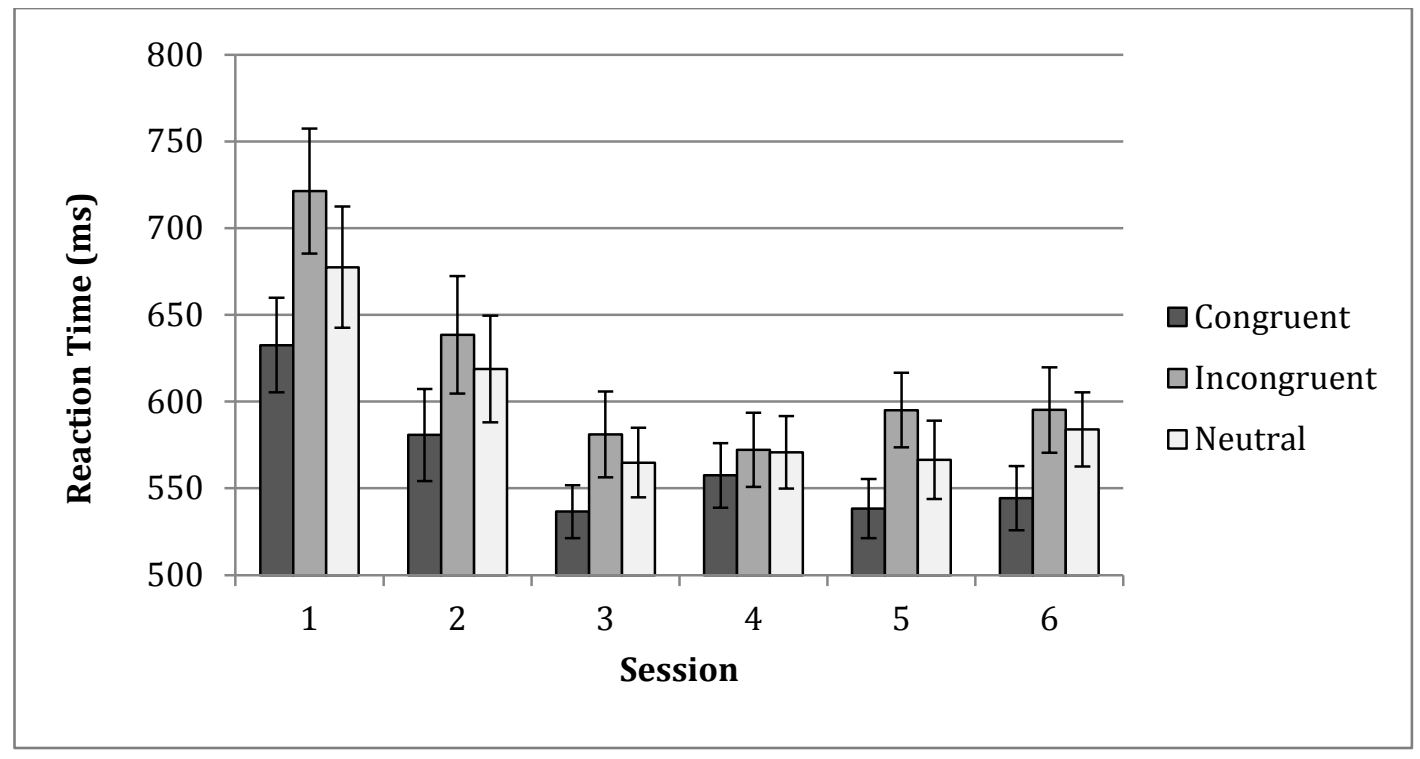

Figure 11. Mean RT in Local-Global with global dimension focus for congruent, incongruent, and neutral trials across six practice sessions. Error bars represent the standard error.

Interference scores (RT). Interferences scores were submitted to 2 (dimension: local vs. global) $\times 6$ (session) repeated measures ANOVA. Results revealed a significant 


\section{PLASTICITY OF THREE SUB-FUNCTIONS OF INHIBITION}

linear session effect, $F(1,23)=6.13, p=.02, \eta^{2}=.21$, suggesting incremental reduction in interference scores overall. However, there was also a significant session $\times$ dimension interaction, $F(5,115)=4.40, p=.001, \eta^{2}=.16$ (see Figure 12). In order to tease apart this interaction, one-way (session) ANOVAs were run separately for the local and global dimensions.

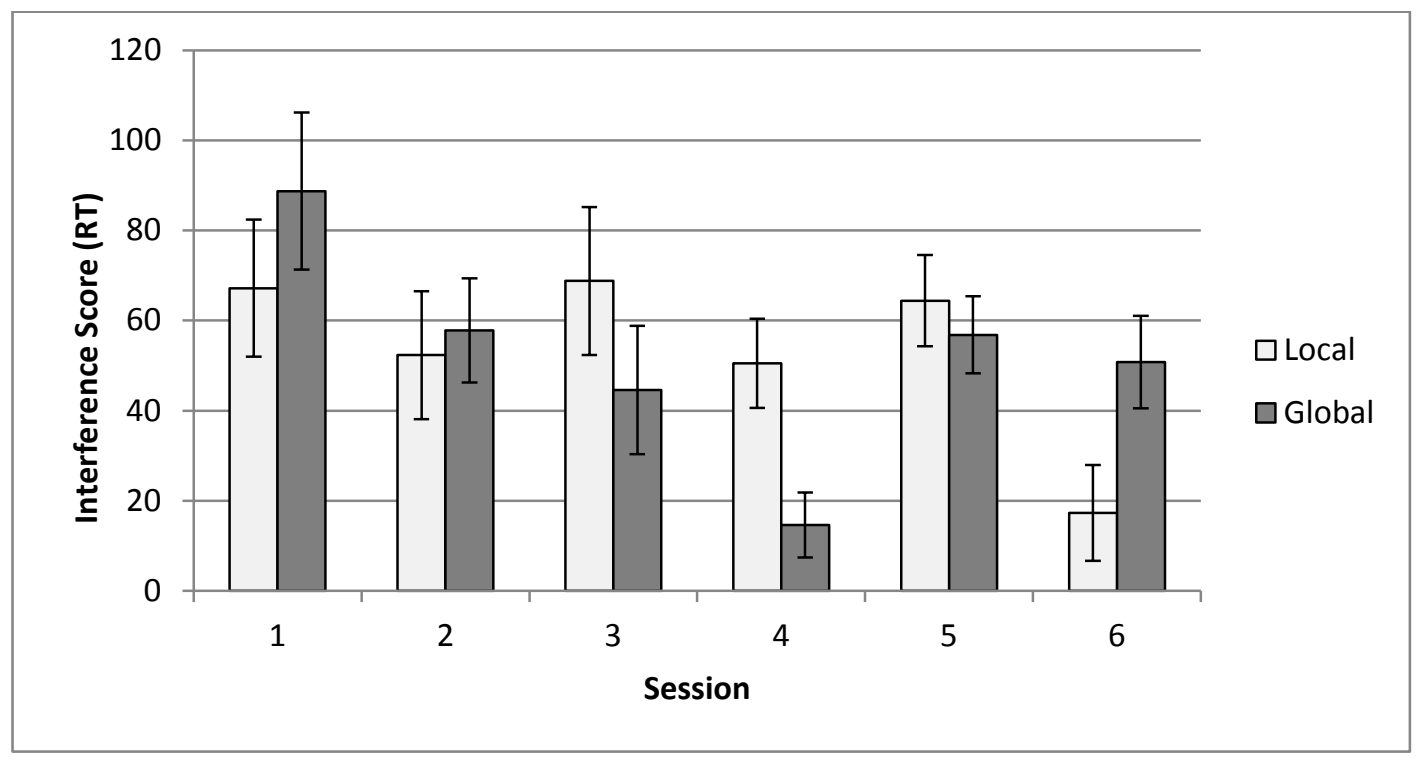

Figure 12. RT interference scores in Local-Global separately for local and global dimension focus. Error bars represent the standard error.

For the local dimension, there was a significant linear session effect, $F(1,23)=$ 7.08, $p=.01, \eta^{2}=.24$ (linear slope $=-6.61$ ). Visual inspection of Figure 12, however, suggests that this effect may be carried by session 6 . To evaluate the role of session 6 in driving the session effect in the local dimension it was removed and the analysis was rerun. After removing session 6, the linear session effect disappeared $(p=.80)$.

For the global dimension, the session effect was approaching significance in linear contrast, $F(1,23)=3.80, p=.06, \eta^{2}=.14$ (linear slope $=-6.36$ ), and significant in quadratic contrast, $F(1,23)=20.96, p<.001, \eta^{2}=.48$. After removing session 6 , as was done in the local dimension, the session effect reached significance in the linear contrast, 


\section{PLASTICITY OF THREE SUB-FUNCTIONS OF INHIBITION}

$F(1,23)=7.48, p=.01, \eta^{2}=.25$, and stayed significant in the quadratic contrasts, $F(1,23)=16.45, p<.001, \eta^{2}=.42$

Taken together, the significant session by dimension interaction in the RT interference scores is likely due to the incremental reduction in global interference scores and the relatively stable local interference scores across at least five of the six retest practice sessions. This suggests that with practice older adults are able to improve upon their ability to keep to-be-ignored information from disrupting their performance. This effect was shown to be most pronounced when the interfering information originated from the local - as opposed to the global - features of a stimulus.

N-Back. Overall accuracy scores were submitted to a 3 (condition: 1-Back, 2-Back, 3-Back) × 6 (session) repeated measures ANOVA. There was a significant session effect in both linear, $F(1,23)=39.56, p<.001, \eta^{2}=.63$, and quadratic contrasts, $F(1,23)=8.14, p=.01, \eta^{2}=.26$ (see Figure 13 )

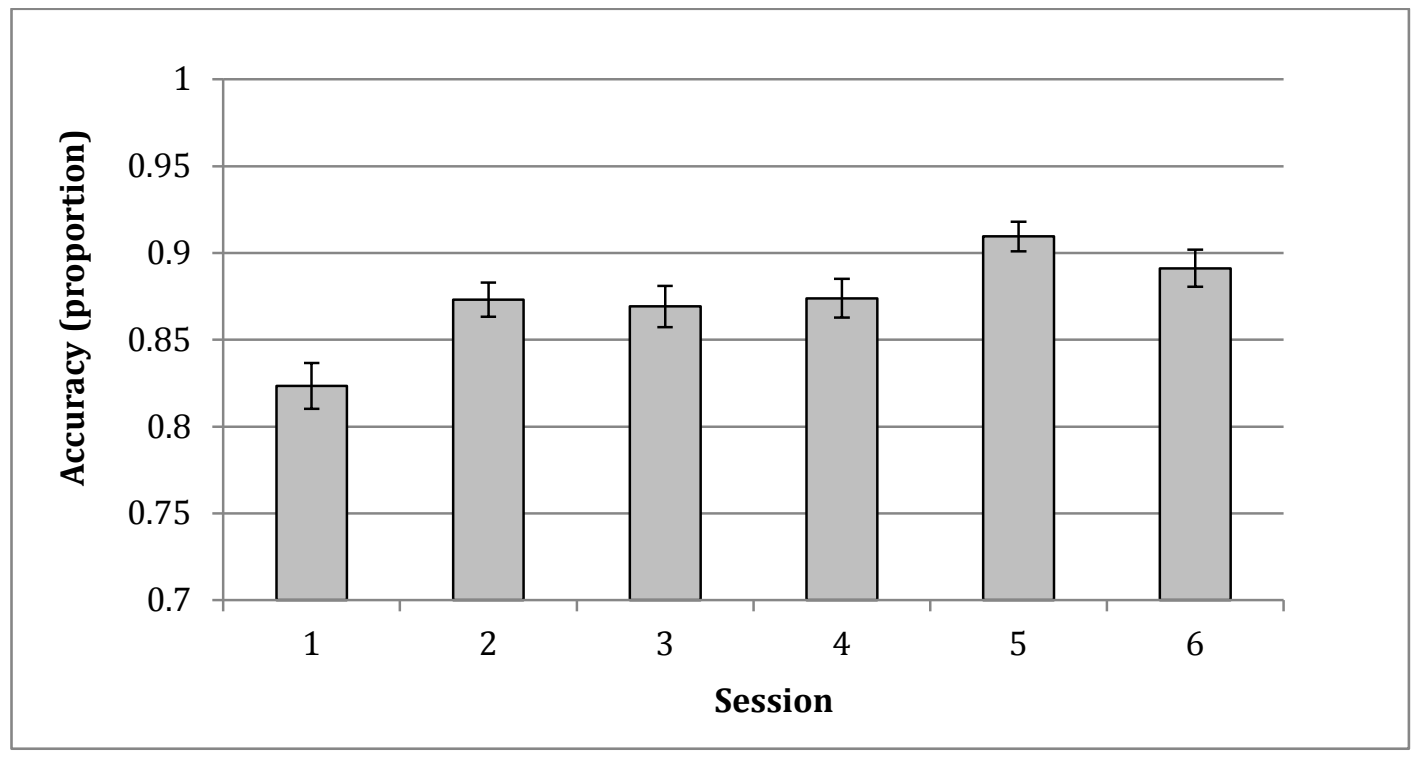

Figure 13. Proportion of accurate responses in N-Back - collapsed across condition across six practice sessions. Error bars represent the standard error. 


\section{PLASTICITY OF THREE SUB-FUNCTIONS OF INHIBITION}

There was also a main effect of condition, $F(2,46)=97.78, p<.001, \eta^{2}=.81$. Specifically, accuracy significantly worsened from 1-Back $(M=.95, S D=.04)$ to 2-Back $(M=.85, S D=.07)$ to 3 -Back $(M=.81, S D=.05)$, all $p s<.001$. Both main effects were qualified by a significant session $\times$ condition interaction, $F(10,230)=1.97, p=.04$, $\eta^{2}=.08$ (see Figure 14). To further explore this interaction, repeated measure (session) ANOVAs were run for each condition separately. The linear effect was significant for all three N-Back conditions, all $p s \leq .001$; however, the quadratic session effect was only significant for the 2-Back condition, $F(1,23)=9.08, p=.01, \eta^{2}=.28$. The linear regression slope was 0.01 for the 1-Back and 2-Back conditions, and 0.02 for the 3-Back condition. For the 2-Back condition only, the quadratic curve analysis (specifying the slope of initial portion of the curve) was examined. This slope was 0.05. Taken together, these slope analyses suggest slightly better incremental improvement in the most difficult condition (i.e., 2-Back and 3-Back).

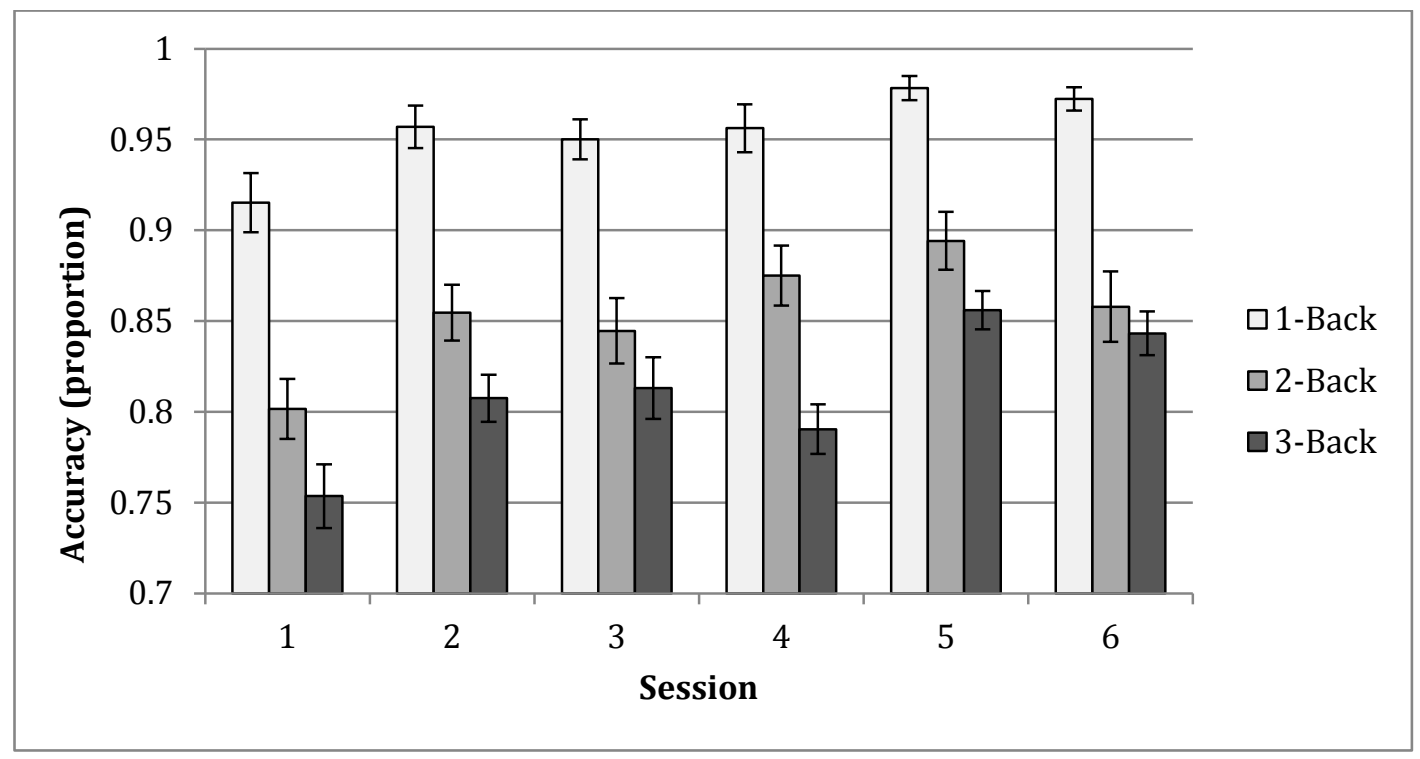

Figure 14. Proportion of accurate responses in N-Back for 1-Back, 2-Back and 3-Back conditions across six practice sessions. Error bars represent the standard error. 


\section{PLASTICITY OF THREE SUB-FUNCTIONS OF INHIBITION}

Go-No Go. The false alarm rate was submitted to a 6 (session) repeated measures ANOVA. This analysis revealed significant session effects in linear, $F(1,23)=13.20$, $p=.001, \eta^{2}=.37$, and quadratic contrasts, $F(1,23)=20.94, p<.001, \eta^{2}=.48$, indicating a significant reduction in false alarms rates with practice (see Figure 15). However, the miss rates did not change across sessions, $F \mathrm{~s}<1.01$, ps $>.32$ for both linear and quadratic session effects. This suggests that the practice-induced performance improvement (reduced false alarms) was independent of miss rates. As a side note, this also indicates that missed responses (i.e., failing to press the 'Go' button for targets) were likely primarily due to a program limitation and did not seem to get better with practice.

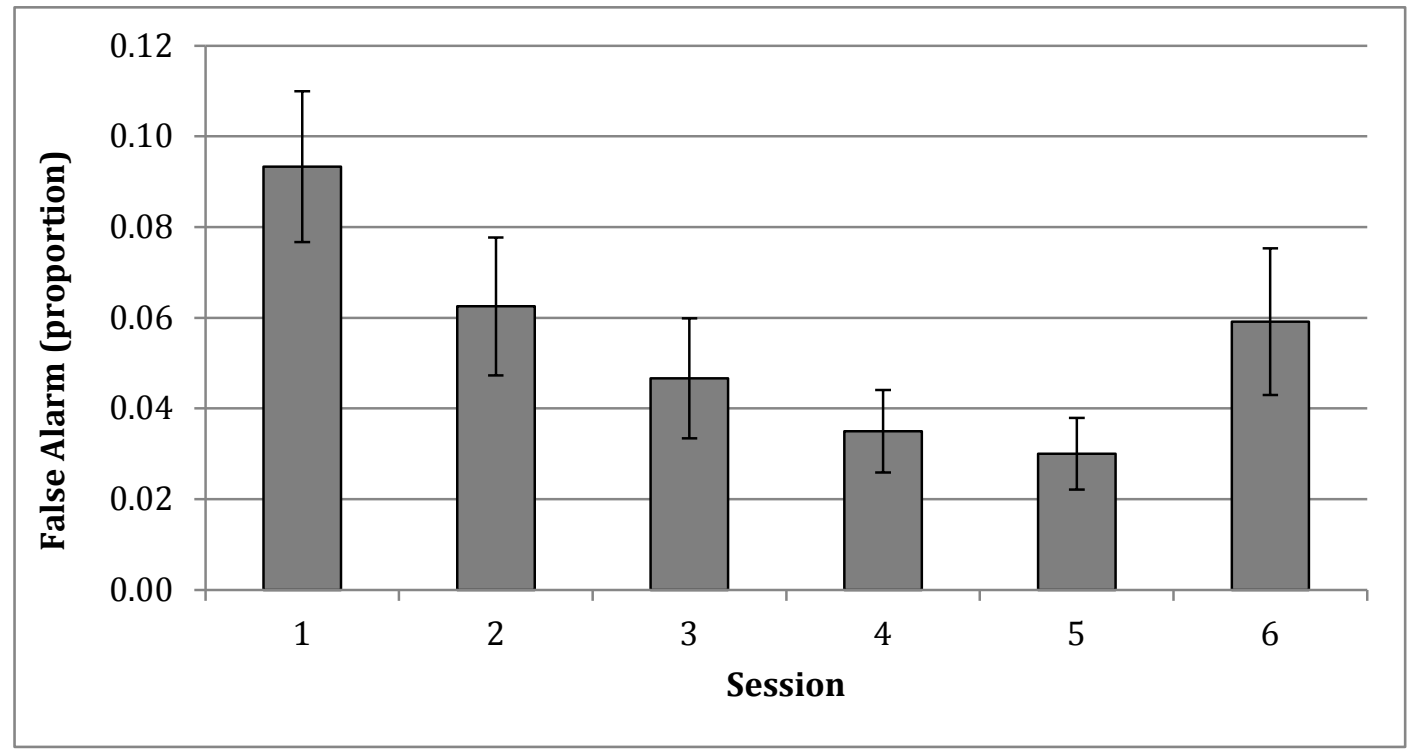

Figure 15. Proportion of false alarms in Go - No Go across six practice sessions. Error bars represent the standard error.

An examination of the miss rates, however, revealed some high values (range: .00 to 1.00). The unsystematically inflated miss rates suggest that E-Prime was not registering the 'spacebar' response on some trials for some participants in a random fashion. In order to conservatively evaluate the effect of this issue on the current data, 


\section{PLASTICITY OF THREE SUB-FUNCTIONS OF INHIBITION}

participant data with miss rates of .30 or higher at any of the six sessions were removed ( $n=10)$ and the analysis was re-run. Using this reduced sample, the 6 (session) repeatedmeasures ANOVA on the false alarm rate revealed the same linear and quadratic contrasts, $p s \leq .02$ (see Figure 16), suggesting that the high miss rates were not biasing our initial findings of a reduced

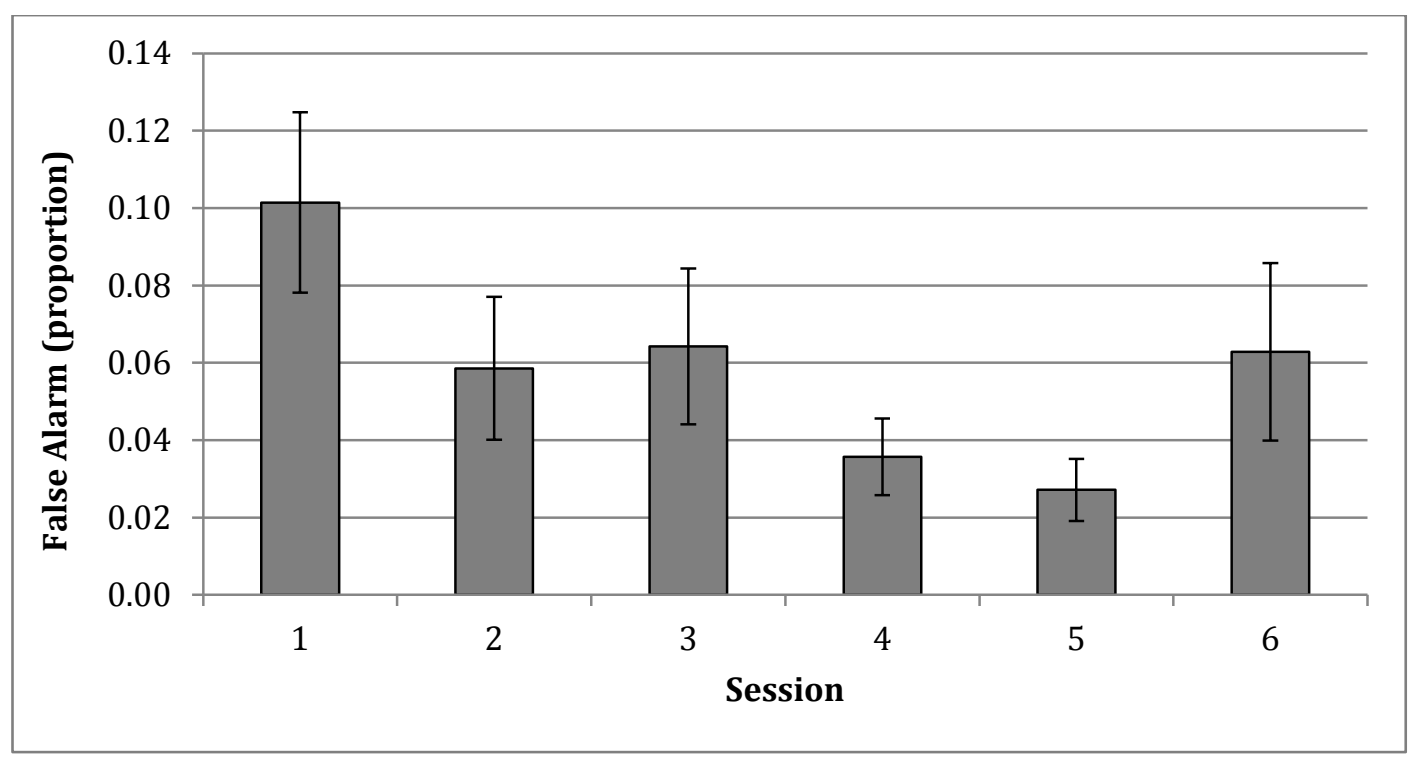

Figure 16. Proportion of false alarms in Go - No Go across six practice sessions with reduced sample $(n=14)$. Error bars represent the standard error.

\section{Transfer Effects}

Transfer effects were established with a significant 2 (session: pretest vs. posttest) $\times 2$ (group: training vs. control) interaction on the outcome variables of interest, and categorized as near-near transfer, near transfer and far transfer tasks.

Near-near transfer. Near-near transfer effects were assessed using parallel versions of the training tasks (i.e., Local-Global, N-Back, and Go - No Go) with digits instead of letters as testing stimuli.

Local-Global. One participant in the training group responded incorrectly to all incongruent trials in the global block at the pretest session, probably due to 


\section{PLASTICITY OF THREE SUB-FUNCTIONS OF INHIBITION}

misunderstanding of the instruction and/or the response key assignment; so, this participant was excluded from the analysis. The overall RT data were submitted to a 2 (session: pretest vs. posttest) $\times 2$ (group: training vs. control) $\times 2$ (dimension: local vs. global) $\times 3$ (trial type: congruent vs. incongruent vs. neutral) mixed model ANOVA. There was a significant session effect, $F(1,45)=20.15, p=.001, \eta^{2}=.31$. Specifically, overall RT was significantly faster at posttest $(M=646.65, S D=254.96)$ relative to pretest $(M=706.18, S D=254.96)$. In addition, there was a main effect of trial type, $F(2,90)=48.50, p<.001, \eta^{2}=.52$. Post hoc paired $t$ tests revealed that congruent trials $(M=636.84, S D=240.46)$ were responded to significantly faster than incongruent $(M=698.32, S D=266.98)$ and neutral trials $(M=694.10, S D=246.54), p s<.001$. The latter two did not differ, $p=.60$. In addition, there was a main effect of dimension, $F(1,45)=5.33, p=.03, \eta^{2}=.11$. Specifically, trials in the local dimension $(M=652.48$, $S D=244.01$ ) were responded to significantly faster than those in the global dimension $(M=700.36, S D=274.46)$, which supports a local precedence effect.

There was also a significant trial type $\times$ dimension interaction, $F(2,90)=6.18$, $p=.003, \eta^{2}=.12$. To tease this apart, the local and global dimensions were compared for each trial type separately using paired $t$ tests. Results revealed that the difference between the local and global dimension was only significant for the incongruent trials, $t(46)=3.33, p=.002$ (all other $p s>.06$ ). In particular, the incongruent trials in the local condition $(M=663.14, S D=255.29)$ were responded to significantly faster than in the global condition $(M=733.50, S D=296.43)$, suggesting that the to-be-ignored dimension was more disruptive when it was coming from the local dimension. This also supports a local precedence effect. 


\section{PLASTICITY OF THREE SUB-FUNCTIONS OF INHIBITION}

Last, the important session $\times$ group interaction was significant, $F(1,45)=8.93$, $p=.01, \eta^{2}=.17$. Follow-up paired $t$ tests revealed that the training group showed significantly smaller RTs at posttest $(M=578.76, S D=103.91)$ relative to pretest $(M=679.35, S D=146.94), p<.001$; however, the control group did not show any differences (posttest: $M=711.72, S D=332.65$; pretest: $M=731.89, S D=328.55$ ), $p=.15$ (see Figure 17). No other effects were significant, $p s>.13$. Following the analyses on the practice effect data, the change in interference scores from pretest to posttest were next explored.

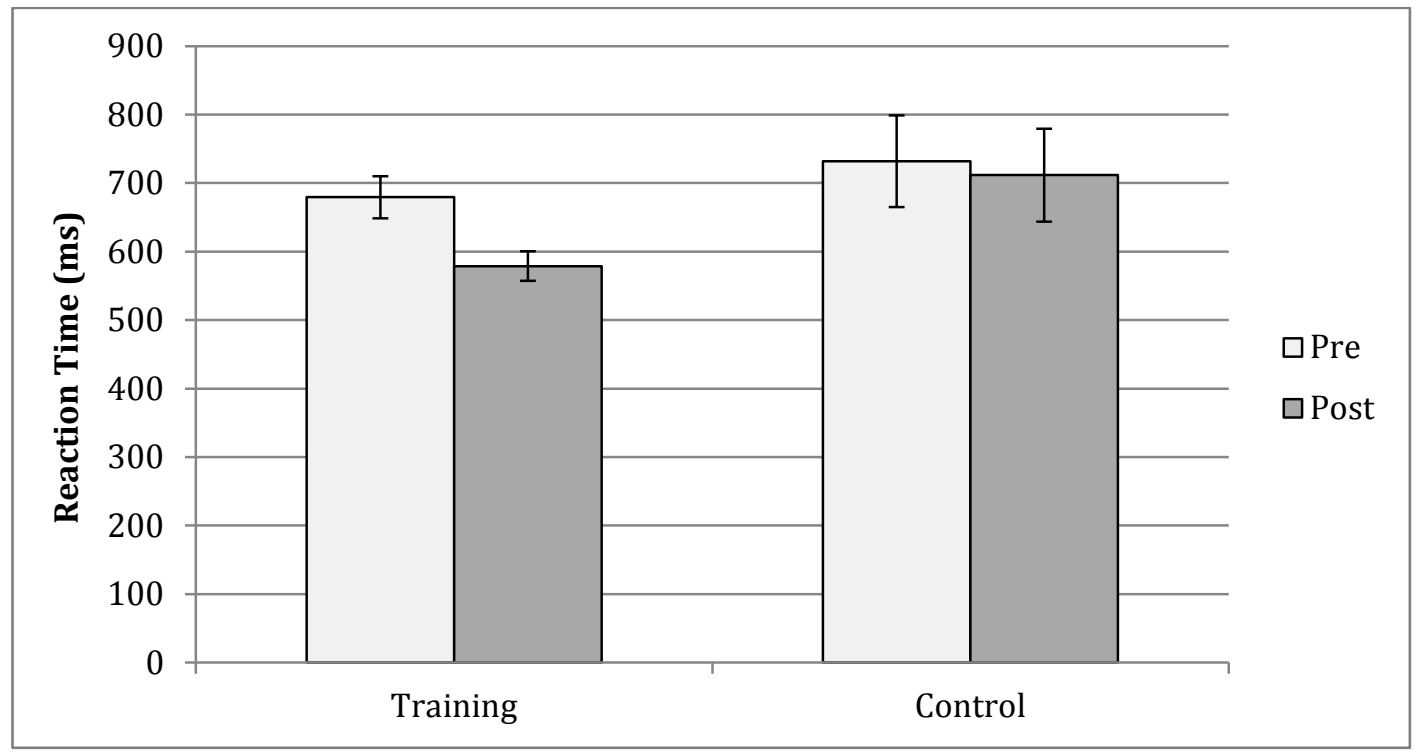

Figure 17. Overall RT in Local-Global at pretest and posttest sessions separately for Training $(n=23)$ and Control $(n=24)$. Error bars represent the standard error.

Interference score (RT). Interference scores (i.e., incongruent RT - congruent RT) were submitted to a 2 (dimension) $\times 2$ (session) $\times 2$ (group) mixed model ANOVA. There was a main effect of session, $F(1,45)=3.94, p=.05, \eta^{2}=.08$, with lower interference at posttest $(M=54.94, S D=47.01)$ relative to pretest $(M=68.03$, $S D=42.86)$. There was also a main effect of dimension, $F(1,45)=14.25, p<.001$, $\eta^{2}=.24$, with lower interference for local $(M=39.06, S D=40.91)$ than for global 


\section{PLASTICITY OF THREE SUB-FUNCTIONS OF INHIBITION}

dimension $(M=83.90, S D=39.06)$. Last, the important session $\times$ group interaction was significant, $F(1,45)=4.16, p=.05, \eta^{2}=.09$ (see Figure 18). Specifically, the training group showed significant reductions in interference from pretest $(M=70.59, S D=45.33)$ to posttest $(M=43.45 ; S D=26.67), p=.01$, while the control group showed no differences (pretest: $M=65.57, S D=41.18$; posttest: $M=65.95, S D=59.00$ ), $p=.97$. This finding supports the presence of near-near transfer to the Local-Global task in older adults. No other effects were significant, $p \mathrm{~s}>.31$.

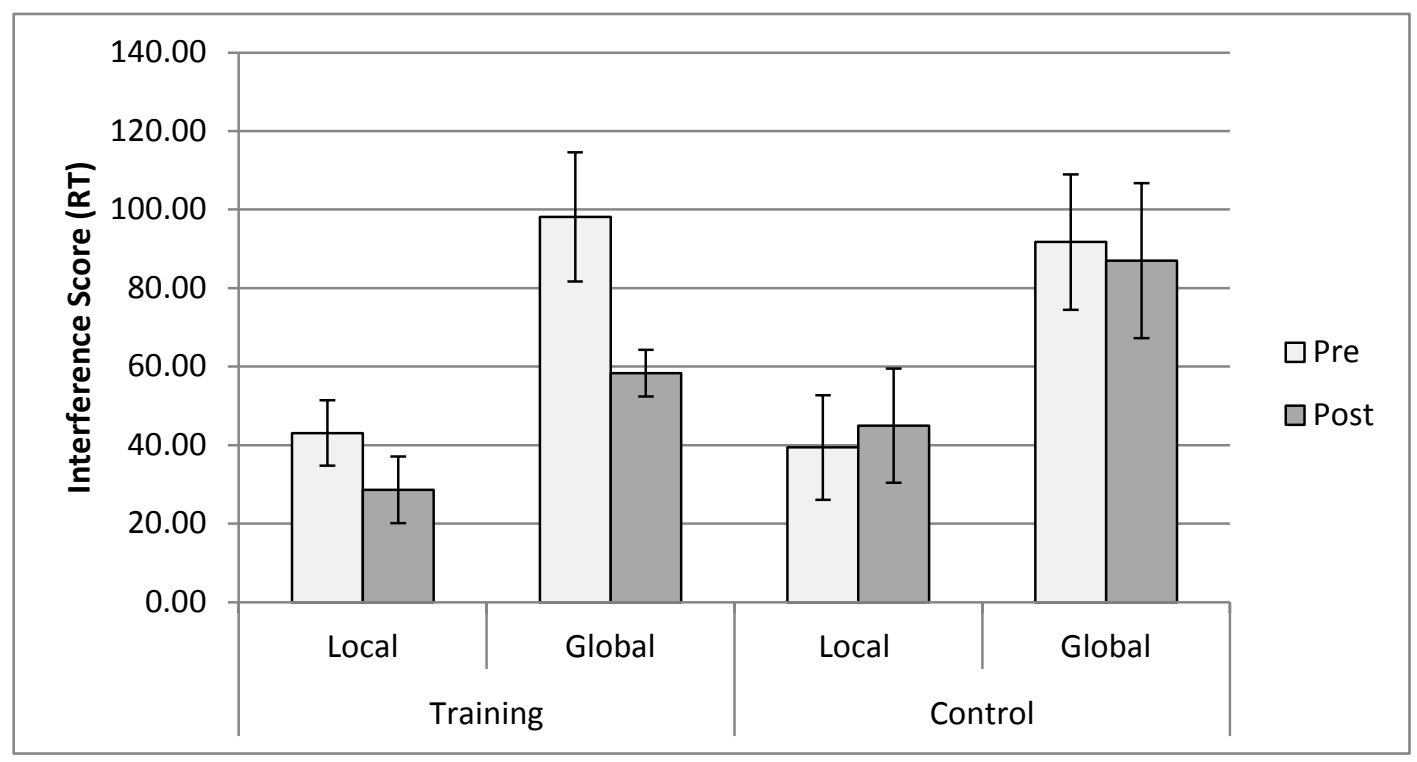

Figure 18. RT interference scores in Local-Global for local and global dimension focus at pretest and posttest sessions separately for Training $(n=23)$ and Control $(n=24)$. Error bars represent the standard error.

Even though the 3-way interaction was not significant, the retest practice effects were shown to be particularly robust during global dimension focus; therefore, the global interference scores were examined, in particular, to explore whether there was any evidence of transfer specifically during global dimension focus.

Global interference score (RT). Interferences scores from the global block were submitted to a 2 (session) $\times 2$ (group) mixed model ANOVA. There was a main effect of 


\section{PLASTICITY OF THREE SUB-FUNCTIONS OF INHIBITION}

session $F(1,45)=4.18, p=.05, \eta^{2}=.09$, with lower interference at posttest $(M=72.94$, $S D=72.54)$ relative to pretest $(M=94.86, S D=81.04)$. The important session $\times$ group interaction did not reach significance, $p=.12$; however, visual inspection of the data reveal that the pattern is in the right direction (see Figure 18). To follow this up, paired $t$ tests were run to compare pretest to posttest performance for the training and control groups separately. These results revealed that, for the training group, the interference scores at posttest $(M=58.31, S D=28.52)$ were significantly smaller than at pretest $(M=98.10, S D=78.98), p=.02$, while there was no difference for the control group (posttest: $M=86.97, S D=96.58$; pretest: $M=91.75, S D=84.54$ ), $p=.75$. Due to a lack of significant interaction, these findings must be interpreted with caution. ${ }^{3}$

$\boldsymbol{N}$-Back. To assess near-near transfer as evidenced with the N-Back task, proportions of accurate responses for each condition were submitted to a 2 (group: training vs. control) $\times 2$ (session: pre vs. post) $\times 4$ (condition: 0-Back, 1-Back, 2-Back, 3-Back) mixed model ANOVA. There was a main effect of session, $F(1,44)=16.55$, $p<.001, \eta^{2}=.27$, whereby overall accuracy was significantly higher at posttest $(M=.91$, $S D=.05)$ relative to pretest $(M=.88, S D=.05)$. There was also a main effect of condition, $F(3,132)=139.15, p<.001, \eta^{2}=.76$. Post hoc paired $t$ tests showed that accuracy continuously dropped from 0 -Back $(M=.99, S D=.03)$ to 1-Back $(M=.96$, $S D=.05)$ to 2 -Back $(M=.84, S D=.09)$ to 3-Back $(M=.79, S D=.07)$, all $p s<.001$. Qualifying these two main effects, there was a significant session $\times$ condition interaction, $F(3,132)=3.96, p=.01, \eta^{2}=.08$. To follow up this interaction, paired $t$ tests were run to

\footnotetext{
${ }^{3}$ No significant effects were found for the same ANOVA run on the local interference scores (all $p s>.19$ ), and similar follow-up $t$ tests revealed no pretest to posttest change for either the training $(p=.10)$ or the control group $(p=.79)$.
} 


\section{PLASTICITY OF THREE SUB-FUNCTIONS OF INHIBITION}

compare pre and posttest performance for each condition separately. Posttest accuracy was significantly greater than pretest for 2-Back, $p=.01$, and 3-Back, $p<.001$, but not for the 0 -Back or 1 -Back conditions, $p s \geq .11$. The results stayed the same after Bonferroni corrections for multiple comparisons (see Figure 19).

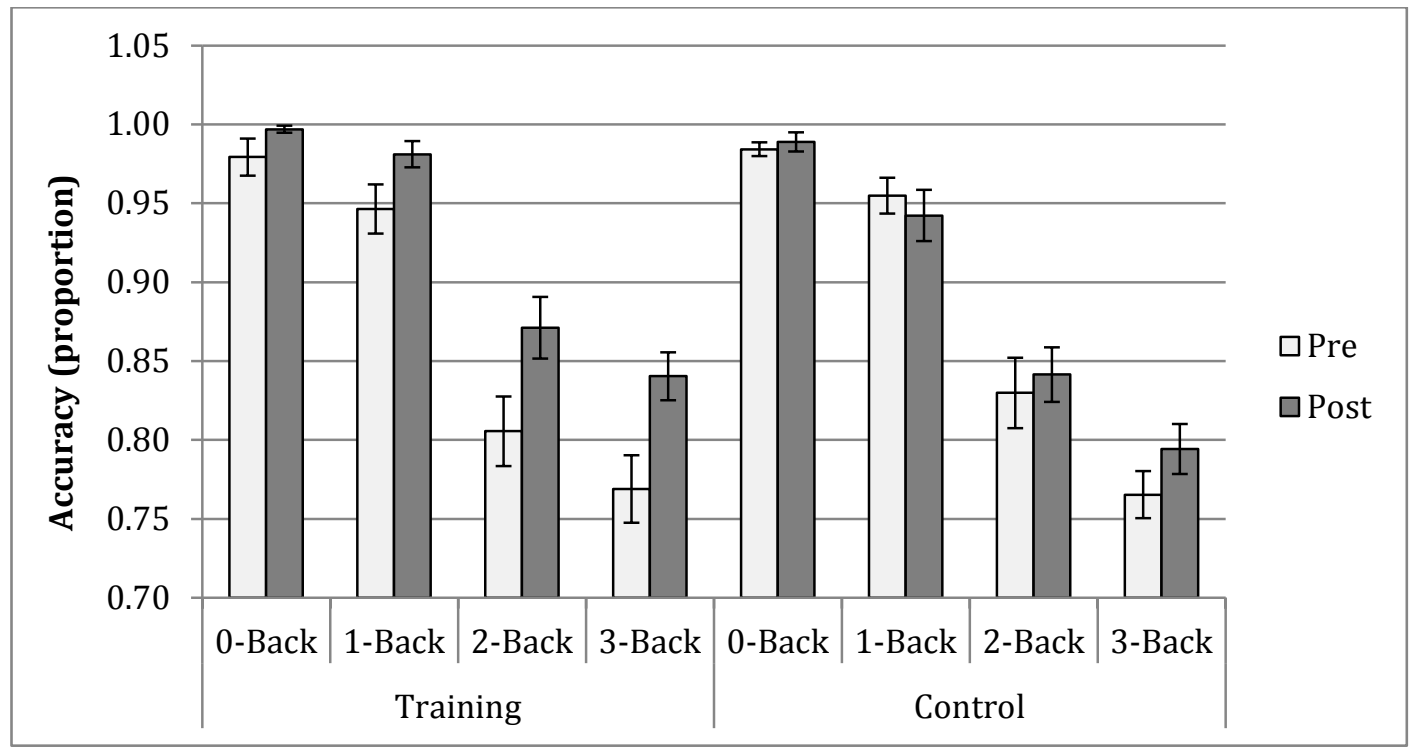

Figure 19. Proportion of accurate responses in N-Back for 0-Back, 1-Back, 2-Back, and 3-Back conditions at pretest and posttest sessions separately for Training and Control. Error bars represent the standard error.

Importantly, there was a significant session $\times$ group interaction, $F(1,44)=8.49$, $p=.01, \eta^{2}=.16$. Paired sample $t$ tests, run separately for each group, showed that the training group performed more accurately at posttest $(M=.92, S D=.05)$ relative to pretest $(M=.88, S D=.05), p=.002$, while the control group showed no difference (pretest: $M=.88, S D=.04$; posttest: $M=.89, S D=.05$ ), $p=.33$, suggesting the presence of near-near transfer (see Figure 20). No other effects were significant, all ps > .35. 


\section{PLASTICITY OF THREE SUB-FUNCTIONS OF INHIBITION}

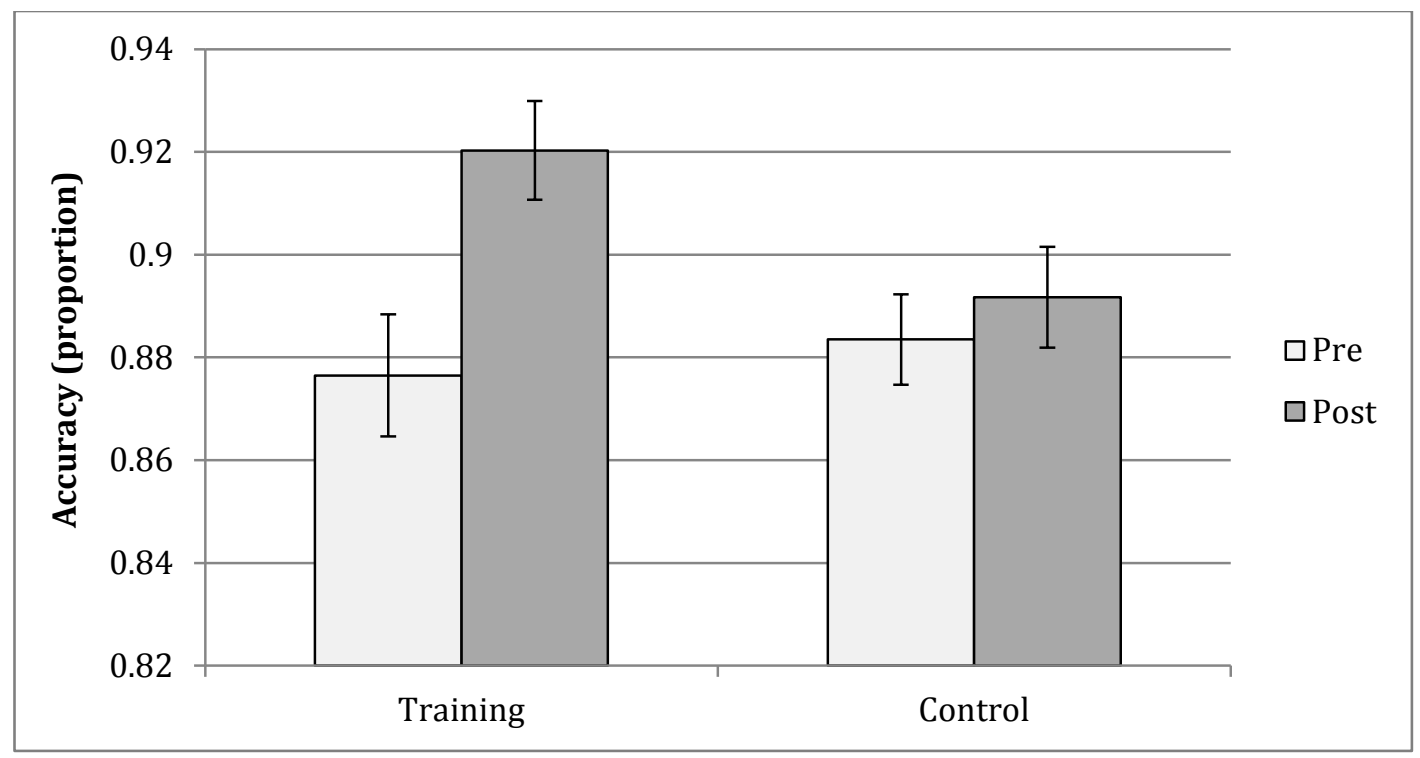

Figure 20. Proportion of accurate responses in N-Back - collapsed across condition - at pretest and posttest sessions separately for Training and Control. Error bars represent the standard error.

Go-No Go. The proportion of false alarms committed during the Go - No Go task were submitted to a 2 (group: training vs. control) $\times 2$ (session: pre vs. post) mixed model ANOVA. There was a significant session effect, $F(1,46)=16.83, p<.001, \eta^{2}=.27$. In particular, significantly fewer false alarms were committed at posttest $(M=.03$, $S D=.06)$ relative to pretest $(M=.07, S D=.09)$. This session effect was qualified by a session $\times$ group interaction, $F(1,46)=8.21, p=.006, \eta^{2}=.15$ (see Figure 21). Post hoc $t$ tests showed that only the training group showed a reduced false alarm rate at posttest $(M=.02, S D=.03)$ as compared to pretest $(M=.08, S D=.09), p=.001$. The control group showed equivalent false alarm rates at posttest $(M=.05, S D=.08)$ and pretest $(M=.06, S D=.09), p=.20$. There was no main effect of group, $F(1,46)=.02, p=.89$, $\eta^{2}=.00$ 


\section{PLASTICITY OF THREE SUB-FUNCTIONS OF INHIBITION}

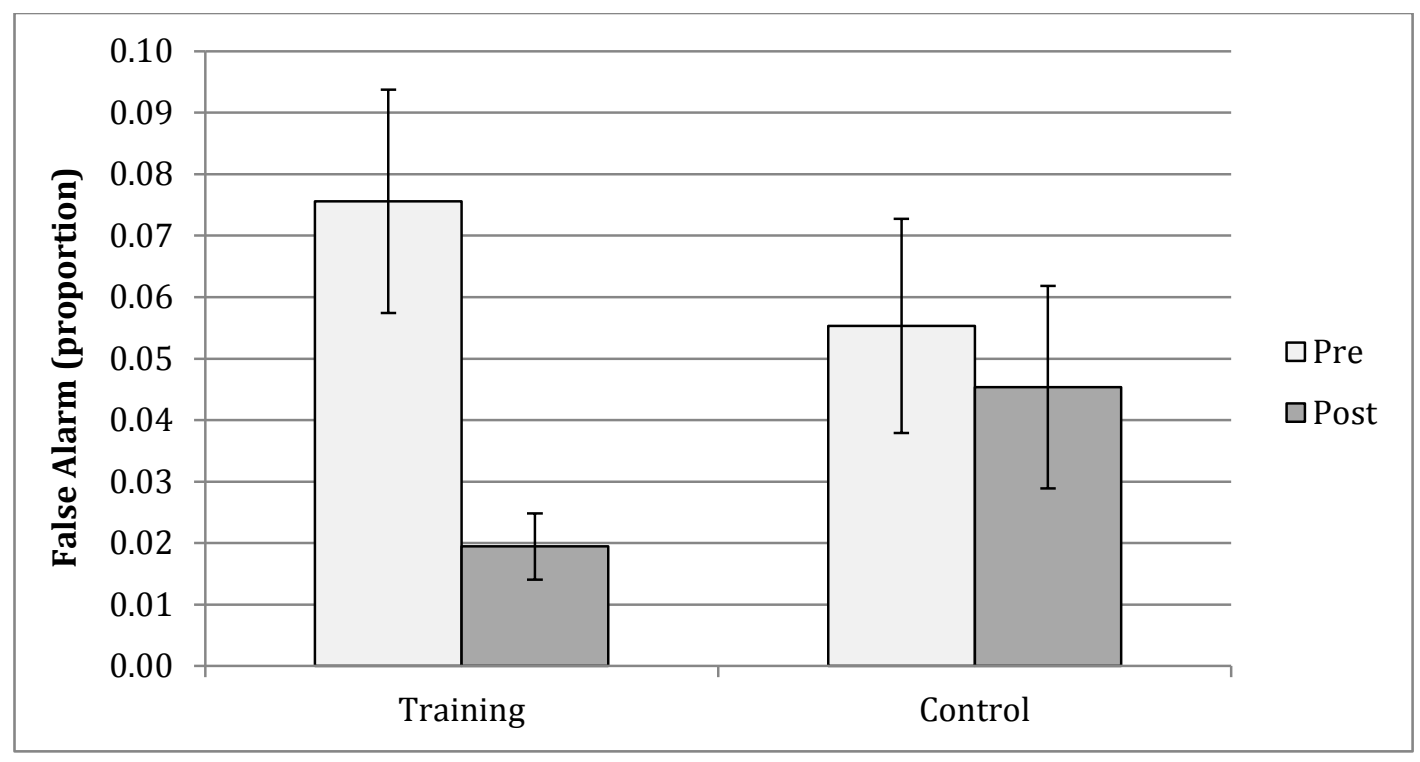

Figure 21. Proportion of false alarms in Go - No Go at pretest and posttest sessions separately for Training and Control. Error bars represent the standard error.

The above analyses were run again after removing individuals with more than a $30 \%$ miss rate ( $n=18$ for training, $n=17$ for control). The results were the same as with the full sample. Specifically, there was a significant session effect, $F(1,33)=13.64$, $p=.001, \eta^{2}=.29$, with fewer false alarms committed at posttest $(M=.04, S D=.01)$ relative to pretest $(M=.08, S D=.02)$. In addition, there was a significant session $\times$ group interaction, $F(1,33)=7.82, p=.01, \eta^{2}=.19$ (see Figure 22). Post hoc $t$ tests were run separately for each group. The training group committed fewer false alarms at posttest $(M=.02, S D=.03)$ as compared to pretest $(M=.09, S D=.10), p=.001$. In contrast, the control group showed the same false alarm rate at posttest $(M=.06, S D=.09)$ and pretest $(M=.07, S D=.10), p=.39$. There was no main effect of group, $F(1,33)=.03, p=.87$, $\eta^{2}=.001$. These follow-up analyses suggest that the near-near transfer effect reported with the full sample was a true effect and not driven by the high miss rates in the data. 


\section{PLASTICITY OF THREE SUB-FUNCTIONS OF INHIBITION}

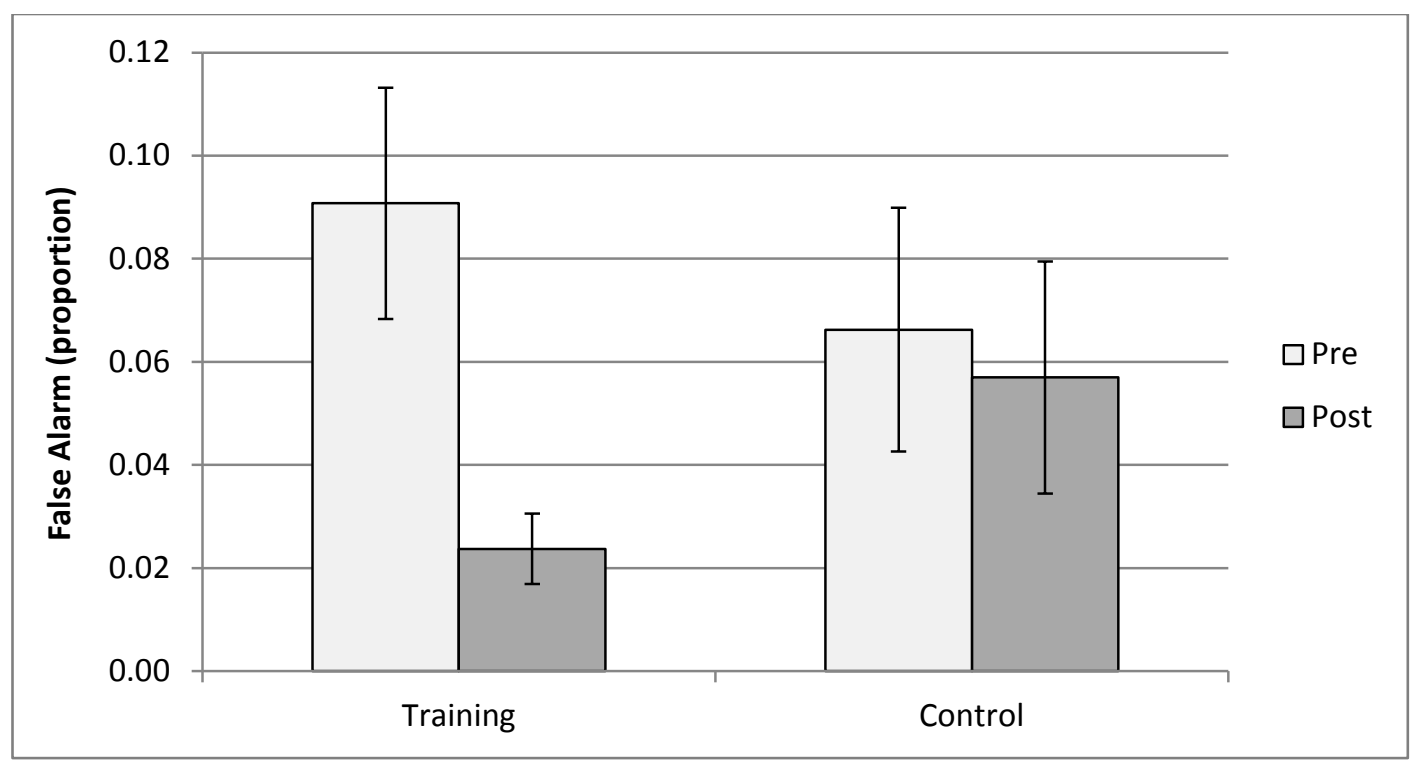

Figure 22. Proportion of false alarms in Go - No Go at pretest and posttest sessions separately for Training $(n=18)$ and Control $(n=17)$ - with reduced samples. Error bars represent the standard error.

Near transfer. Near transfer effects were assessed using tasks that were structurally different from the training tasks, but known in the literature to tap into the same sub-functions of inhibition. In particular, Reading with Distraction, Directed Forgetting and Stroop tasks were used to evaluate near transfer effects.

Reading with Distraction. Passage reading speed and accuracy on the multiplechoice questions were both examined to explore the presence of near transfer effects.

Passage reading speed. The average speed at which a passage was read (in seconds) was calculated separately for high versus low distraction conditions, for each participant and at each session. None $(0 \%)$ of the RTs for passage reading speed were beyond 2.5 SDs away from the cell means. For one participant, the passage reading speed for one (of two) high distracting passages was missing at pretest due to a technical glitch; therefore, only a single score was inputted into their high distracting condition RT at 


\section{PLASTICITY OF THREE SUB-FUNCTIONS OF INHIBITION}

pretest. Reading speed distraction scores were calculated by subtracting the RT of low distracting from that of high distracting passages.

The reading speed distraction scores were submitted to a 2 (session) $\times 2$ (group) mixed model ANOVA. The critical session $\times$ group interaction was not significant, $F(1,46)=.16, p=.69, \eta^{2}=.003$, indicating no near transfer to reading speed in the Reading with Distraction task (see Table 10). No other effects were significant, all ps $>.54$.

Multiple-choice performance accuracy. First, the overall accuracy on the multiple-choice questions was submitted to a 2 (session) $\times 2$ (condition) $\times 2$ (group) mixed model ANOVA. Although there were main effects of session (pre $[M=.81$, $S D=.09]>$ post $[M=.71, S D=.11], p<.001$ ), condition (low $[M=.83, S D=.11]>$ high $[M=.68, S D=.11], p<.001$ ), and group (training $[M=.78, S D=.08]>$ control $[M=.73, S D=.08], p=.02)$, there were no significant interactions, all $p s>.45$, indicating a lack of transfer effects in terms of overall accuracy performance.

Last, the proportion of distractor intrusions in the multiple-choice responses for the high distracting passage was examined. This proportion was submitted to a 2 (session) $\times 2$ (group) mixed model ANOVA. No effects were significant, $p$ s $>.23$ (see Table 10). 


\section{PLASTICITY OF THREE SUB-FUNCTIONS OF INHIBITION}

Table 10

Pretest - Posttest Performance in Reading with Distraction Separately for Training and No Training Control

\begin{tabular}{|c|c|c|c|c|c|c|}
\hline \multirow{2}{*}{ Variable } & \multicolumn{3}{|c|}{$\begin{array}{l}\text { Training } \\
(n=24)\end{array}$} & \multicolumn{3}{|c|}{$\begin{array}{l}\text { No Training Control } \\
(n=24)\end{array}$} \\
\hline & Pre & Post & $d$ & Pre & Post & $d$ \\
\hline \multicolumn{7}{|l|}{ Passage reading speed } \\
\hline Low distraction & $\begin{array}{l}71.57 \\
(17.39)\end{array}$ & $\begin{array}{l}68.57 \\
(13.72)\end{array}$ & .19 & $\begin{array}{l}70.71 \\
(19.80)\end{array}$ & $\begin{array}{l}68.73 \\
(18.00)\end{array}$ & .10 \\
\hline High distraction & $\begin{array}{l}100.37 \\
(34.91)\end{array}$ & $\begin{array}{l}95.60 \\
(33.22)\end{array}$ & .14 & $\begin{array}{l}95.94 \\
(35.57)\end{array}$ & $\begin{array}{l}93.59 \\
(40.13)\end{array}$ & .06 \\
\hline Distraction score & $\begin{array}{l}28.80 \\
(20.91)\end{array}$ & $\begin{array}{l}27.03 \\
(24.51)\end{array}$ & .08 & $\begin{array}{l}25.23 \\
(19.76)\end{array}$ & $\begin{array}{l}24.85 \\
(24.39)\end{array}$ & .02 \\
\hline \multicolumn{7}{|l|}{ Multiple-choice } \\
\hline Low distraction & $\begin{array}{l}.90 \\
(.10)\end{array}$ & $\begin{array}{l}.80 \\
(.14)\end{array}$ & .82 & $.85(.13)$ & $.77(.15)$ & .57 \\
\hline High distraction & $\begin{array}{l}.77 \\
(.11)\end{array}$ & $\begin{array}{l}.67 \\
(.16)\end{array}$ & .73 & $.71(.13)$ & $.59(.15)$ & .85 \\
\hline Distractor intrusion & $\begin{array}{l}.21 \\
(.09)\end{array}$ & $\begin{array}{l}.22 \\
(.13)\end{array}$ & .09 & $.21(.12)$ & $.26(.15)$ & .37 \\
\hline
\end{tabular}

Note. Standard deviations are in parentheses. Passage reading speed for high and low distraction conditions was measured in seconds; Distraction score was indexed by the difference in reading speed between the high and low distraction conditions; Multiple-choice responses denoted by the proportion of correct responses; Distractor intrusion was indexed by the proportion of to-be-ignored words selected during the multiple-choice questions following a high distraction condition passage.

$d=$ Cohen's $d$ effect size calculation for between subjects.

Directed Forgetting. Mean RT data (collected during recognition) was calculated separately for each word type (TBR, TBF, and NEW), session (pretest and posttest), and accuracy level (correct and incorrect). RTs were trimmed by removing those that were 


\section{PLASTICITY OF THREE SUB-FUNCTIONS OF INHIBITION}

2.5 SDs above or below the mean for each cell. Using this criterion, $4.51 \%$ of the trials were removed.

Overall RT. Overall RT for 'new' responses to TBF and NEW words were submitted to a 2 (session) $\times 2$ (group) $\times 2$ (word type: TBF vs. NEW). Only those participants who responded 'new' to a TBF words were included in this analysis $(n=10$ for training; and $n=13$ for control). The only significant effect was a main effect of word type, $F(1,21)=28.64, p<.001, \eta^{2}=.58$. Overall, responding 'new' to a TBF word $(M=1706.63, S D=524.06)$ took significantly longer than responding 'new' to a NEW word $(M=1228.58, S D=271.99)$, indicating inefficient deletion. This effect, however, was not modulated by inhibition training.

Hit rate. The proportion of hits were submitted to a 2 (session) $\times 2$ (group) $\times$ 2 (word type: TBR vs. TBF) mixed model ANOVA. There was a main effect of word type, $F(1,46)=47.58, p<.001, \eta^{2}=.51$. Post hoc $t$ tests revealed the presence of a directed forgetting effect, with significantly higher hits for TBR $(M=.89, S D=.13)$ than TBF words $(M=.76, S D=.17)$. However, no other effects were significant, $p s>.12$, indicating an absence of near transfer effects. Since the false alarm rate was the same for TBR and TBF words within a session, there was no added benefit in calculating and analyzing the discrimination index $\left(\mathrm{P}_{\mathrm{r}}=\right.$ hits - false alarms). Taken together, the current data failed to reveal near transfer of inhibition training to the Directed Forgetting task (see Table 11). 


\section{PLASTICITY OF THREE SUB-FUNCTIONS OF INHIBITION}

Table 11

Pretest - Posttest Performance in Directed Forgetting Separately for Training and No Training Control

\begin{tabular}{|c|c|c|c|c|c|c|}
\hline \multirow[b]{2}{*}{ Variable } & \multicolumn{3}{|l|}{$\begin{array}{l}\text { Training } \\
(n=24)\end{array}$} & \multicolumn{3}{|c|}{$\begin{array}{l}\text { No Training Control } \\
(n=24)\end{array}$} \\
\hline & Pre & Post & $d$ & Pre & Post & $d$ \\
\hline \multicolumn{7}{|l|}{ Reaction time } \\
\hline TBF ('new') & $\begin{array}{l}1837.20^{\gamma} \\
(546.15)\end{array}$ & $\begin{array}{l}1522.20^{\gamma} \\
(746.87)^{\gamma}\end{array}$ & .73 & $\begin{array}{l}1590.55^{¥} \\
(401.94)\end{array}$ & $\begin{array}{l}1705.38^{\ddagger} \\
(922.33)\end{array}$ & .10 \\
\hline NEW ('new') & $\begin{array}{l}1296.57^{\gamma} \\
(399.24)\end{array}$ & $\begin{array}{l}1134.25^{\gamma} \\
(338.05)\end{array}$ & .25 & $\begin{array}{l}1224.57^{¥} \\
(293.53)\end{array}$ & $\begin{array}{l}1224.97^{¥} \\
(327.06)\end{array}$ & .04 \\
\hline \multicolumn{7}{|l|}{ Hit rate } \\
\hline TBR & $.89(.17)$ & $.86(.16)$ & .18 & $.86(.15)$ & $.94(.10)$ & .63 \\
\hline $\mathrm{TBF}$ & $.75(.20)$ & $.77(.20)$ & .10 & $.75(.20)$ & $.77(.20)$ & .10 \\
\hline \multicolumn{7}{|l|}{ False alarm rate } \\
\hline NEW & $.05(.07)$ & $.08(.13)$ & .29 & $.06(.10)$ & $.07(.08)$ & .11 \\
\hline
\end{tabular}

Note. Standard deviations are in parentheses. Reaction time (in ms) for TBF words incorrectly identified as 'new' and NEW words correctly identified as 'new'; Hit rate denoted as the proportion of TBR or TBF words correctly identified as 'old'; False alarm rate denoted as the proportion of NEW words incorrectly identified as 'old'.

${ }^{x} n=10 .{ }^{¥} n=13$.

$d=$ Cohen's $d$ effect size calculation for between subjects.

Stroop. To calculate Stroop interference scores, mean RT was calculated for each trial type separately at each session. Only RTs of correct trials were included in the analyses. Furthermore, outliers - defined as 2.5 SDs above or below the mean for each trial type at each session - were removed from the data set. Using this criterion, $2.66 \%$ of the trials were trimmed. As mentioned previously, three participants presented as being colourblind and two other participants used the number keypad (instead of the keyboard) when responding during the Stroop task. The data were re-analyzed three times, once excluding the colourblind participants, once excluding the number keypad participants, 


\section{PLASTICITY OF THREE SUB-FUNCTIONS OF INHIBITION}

and once excluding both at the same time. In all cases, the results remained unchanged; therefore, the analyses reported below include all participants. For information on the raw data scores, see Table 12.

Table 12

Pretest - Posttest Performance in Stroop Separately for Training and No Training Control

\begin{tabular}{|c|c|c|c|c|c|c|}
\hline \multirow[b]{2}{*}{ Variable } & \multicolumn{3}{|l|}{$\begin{array}{l}\text { Training } \\
(n=24)\end{array}$} & \multicolumn{3}{|c|}{$\begin{array}{l}\text { No Training Control } \\
(n=24)\end{array}$} \\
\hline & Pre & Post & $d$ & Pre & Post & $d$ \\
\hline \multicolumn{7}{|l|}{ Reaction time } \\
\hline Congruent & $\begin{array}{l}906.17 \\
(328.29)\end{array}$ & $\begin{array}{l}793.58 \\
(227.33)\end{array}$ & .40 & $\begin{array}{l}921.55 \\
(287.68)\end{array}$ & $\begin{array}{l}890.07 \\
(251.48)\end{array}$ & .12 \\
\hline Incongruent & $\begin{array}{l}1117.56 \\
(457.68)\end{array}$ & $\begin{array}{l}952.25 \\
(270.96)\end{array}$ & .44 & $\begin{array}{l}1102.36 \\
(322.45)\end{array}$ & $\begin{array}{l}1013.62 \\
(245.03)\end{array}$ & .31 \\
\hline Neutral & $\begin{array}{l}907.78 \\
(294.94)\end{array}$ & $\begin{array}{l}829.58 \\
(240.89)\end{array}$ & .29 & $\begin{array}{l}935.55 \\
(276.74)\end{array}$ & $\begin{array}{l}877.17 \\
(216.90)\end{array}$ & .23 \\
\hline Interference & $\begin{array}{l}209.78 \\
(189.38)\end{array}$ & $\begin{array}{l}122.67 \\
(82.17)\end{array}$ & .60 & $\begin{array}{l}166.81 \\
(88.85)\end{array}$ & $\begin{array}{l}136.44 \\
(93.36)\end{array}$ & .33 \\
\hline \multicolumn{7}{|l|}{ Accuracy } \\
\hline Congruent & $.98(.03)$ & $.99(.02)$ & .39 & $.99(.02)$ & $.99(.02)$ & .00 \\
\hline Incongruent & $.95(.10)$ & $.98(.03)$ & .41 & $.95(.06)$ & $.96(.07)$ & .15 \\
\hline Neutral & $.99(.03)$ & $.98(.03)$ & .33 & $.98(.03)$ & $.98(.02)$ & .00 \\
\hline Interference & $.04(.07)$ & $.01(.02)$ & .58 & $.03(.04)$ & $.02(.05)$ & .22 \\
\hline
\end{tabular}

Note. Standard deviations are in parentheses. RT was denoted in ms. Accuracy was denoted by the proportion of correct responses; Interference scores were indexed by the difference in performance between incongruent and neutral trials. Reaction time (RT) interference = incongruent RT - neutral RT; Accuracy interference $=$ neutral accuracy - incongruent accuracy.

$d$ = Cohen's $d$ effect size calculation for between subjects .

Stroop interference (accuracy). Stroop interference scores were calculated with accuracy (neutral accuracy - incongruent accuracy) and submitted to a 2 (session) $\times$ 


\section{PLASTICITY OF THREE SUB-FUNCTIONS OF INHIBITION}

2 (group) mixed model ANOVA. The critical session $\times$ group interaction was not significant, $F(1,46)=1.80, p=.19, \eta^{2}=.04$, indicating no near transfer to accuracy interference scores in Stroop (see Table 12). No other effects were significant, all ps $>.08$.

Stroop interference (RT). Stroop interference scores were calculated with RT (incongruent RT- neutral RT) and submitted to a 2 (session) $\times 2$ (group) mixed model ANOVA. There was a main effect of session, $F(1,46)=8.41, p=.01, \eta^{2}=.16$. Specifically, RT interference scores were significantly reduced at posttest $(M=129.56$, $S D=87.28)$ relative to pretest $(M=188.30, S D=147.94)$. There was no session $\times$ group interaction, $F(1,46)=1.96, p=.17, \eta^{2}=.04$; however, visual inspection suggest that the interference scores reduced from pretest to posttest for the training group, but not for the control group (see Figure 23).

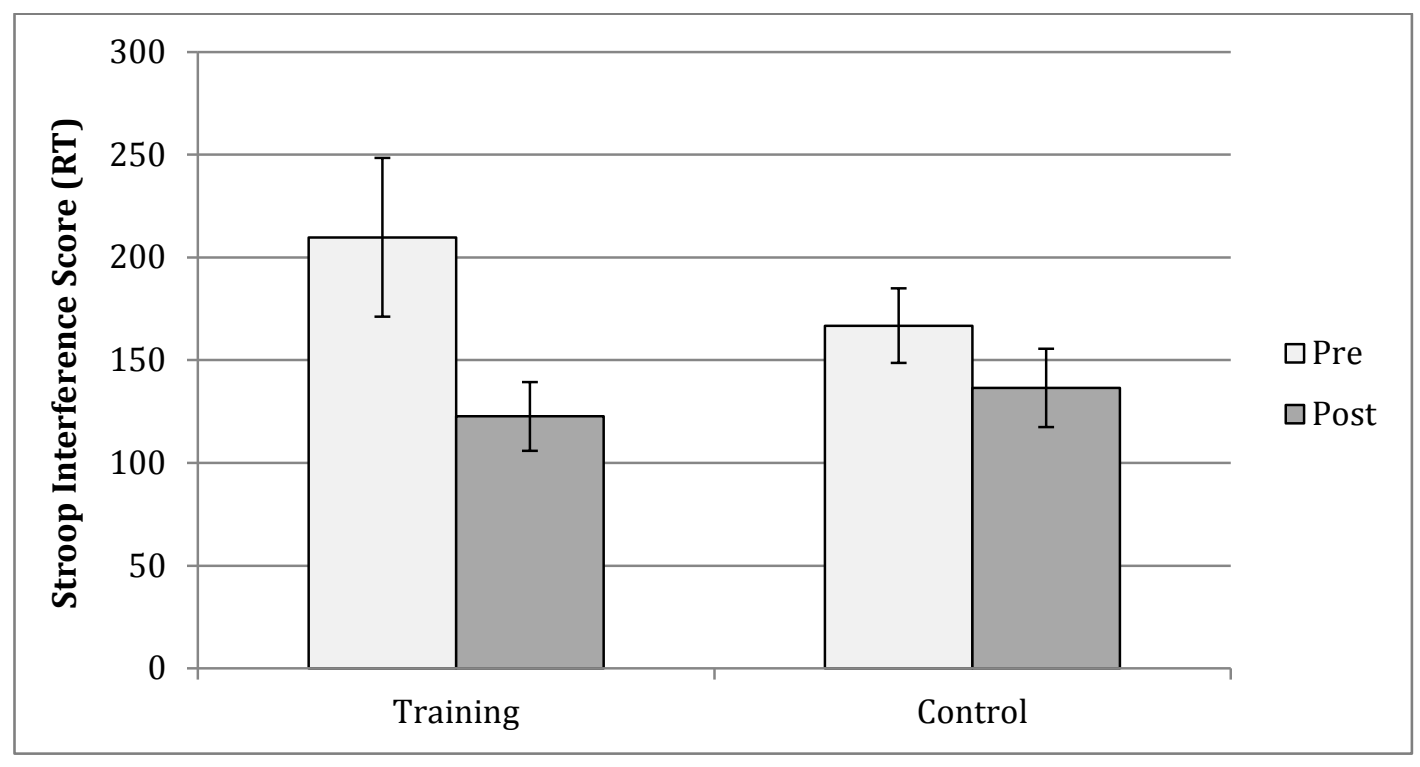

Figure 23. RT interference scores in Stroop at pretest and posttest sessions separately for Training and Control. Error bars represent the standard error.

In order to investigate this possibility, separate paired $t$ tests were run for the training and control groups. The training group showed a significant reduction in Stroop 


\section{PLASTICITY OF THREE SUB-FUNCTIONS OF INHIBITION}

interference from pretest $(M=209.78, S D=189.38)$ to posttest $(M=122.67$, $S D=82.17), p=.024, d=.60$, while the control group showed equivalent pretest $(M=166.81, S D=88.85)$ and posttest $(M=136.44 .78, S D=93.36)$ performance, $p=.12, d=.33$. Due to a lack of significant interaction, however, these findings must be interpreted with caution. No other effect was significant, $p=.61$.

Far transfer. Far transfer effects were assessed using tasks that were dissimilar in structure and tapped different cognitive domains than the tasks used in the training sessions. Here, far transfer of inhibition training to working memory (Corsi Block), episodic memory (Word List Recall), reasoning (Letter Series) and processing speed (Digit Symbol) was explored.

Corsi Block. First, the proportion of accurate responses within a trial was calculated and then averaged across each span length (i.e., 4-span, 5-span, 6-span and 7-span) at each session. These accuracy scores were submitted to a 2 (session) $\times$ 2 (group) $\times(4$ span length) mixed model ANOVA. The only significant effect was a main effect of span length, $F(3,138)=186.62, p<.001, \eta^{2}=.80$. Post hoc $t$ tests revealed that accuracy of the four different span conditions were significantly different from each other: 4 -span $(M=.82, S D=.17)$, 5 -span $(M=.77, S D=.16)$, 6-span $(M=.49, S D=.14)$ and 7-span $(M=.39, S D=.15)$, all $p s<.04$. The difference between span 4 and 5 , however, did not remain significant after correcting for multiple comparisons using Bonferroni corrections $(.05 / 6=.001)$. No other effects were significant $(p s>.18)$, indicating a lack of far transfer (see Table 13).

Following Rowe et al. (2009), overall accuracy performance was also examined as the proportion of trials that were correctly recalled in the right sequence. These 


\section{PLASTICITY OF THREE SUB-FUNCTIONS OF INHIBITION}

accuracy scores were submitted to a 2 (session) x 2 (group) mixed model ANOVA. No effects were significant, $p s>.60$, further supporting an absence of far transfer of inhibition training to the Corsi Block task (see Table 13).

Table 13

Pretest - Posttest Accuracy Performance in Corsi Block Separately for Training and No Training Control

\begin{tabular}{|c|c|c|c|c|c|c|}
\hline \multirow{2}{*}{ Variable } & \multicolumn{3}{|l|}{$\begin{array}{l}\text { Training } \\
(n=24)\end{array}$} & \multicolumn{3}{|c|}{$\begin{array}{l}\text { No Training Control } \\
(n=24)\end{array}$} \\
\hline & Pre & Post & $d$ & Pre & Post & $d$ \\
\hline Span 4 & $.82(.18)$ & $.83(.19)$ & .05 & $.83(.21)$ & $.79(.24)$ & .18 \\
\hline Span 5 & $.76(.16)$ & $.78(.20)$ & .11 & $.73(.25)$ & $.81(.17)$ & .37 \\
\hline Span 6 & $.50(.22)$ & $.53(.17)$ & .15 & $.46(.21)$ & $.45(.20)$ & .05 \\
\hline Span 7 & $.36(.13)$ & $.40(.21)$ & .23 & $.39(.18)$ & $.41(.18)$ & .11 \\
\hline Overall & $.35(.17)$ & $.37(.16)$ & .12 & $.34(.20)$ & $.34(.15)$ & .00 \\
\hline
\end{tabular}

Note. Standard deviations are in parentheses. Span 4/5/6/7 denotes the proportion of item-based correct responses within each span condition; Overall indexed the proportion of entire trials correctly recalled in the right sequence.

$d=$ Cohen's $d$ effect size calculation for between subjects.

Word List Recall. The proportion of correct responses, correct in right sequence, correct in wrong sequence, correct overall, and wrong words were submitted to separate 2 (session) $\times 2$ (group) mixed model ANOVAs. The critical session $\times$ group interaction was not significant for any of the dependent variables (all $p s>.38$ ), indicating no far transfer to episodic memory (see Table 14). No other effects were significant, all ps $>.09$. 


\section{PLASTICITY OF THREE SUB-FUNCTIONS OF INHIBITION}

Table 14

Pretest - Posttest Performance in Word List Recall Separately for Training and No Training Control

\begin{tabular}{|c|c|c|c|c|c|c|}
\hline \multirow[b]{2}{*}{ Variable } & \multicolumn{3}{|l|}{$\begin{array}{l}\text { Training } \\
(n=24)\end{array}$} & \multicolumn{3}{|c|}{$\begin{array}{l}\text { No Training Control } \\
(n=24)\end{array}$} \\
\hline & Pre & Post & $d$ & Pre & Post & $d$ \\
\hline Correct & $.40(.35)$ & $.47(.37)$ & .19 & $.45(.31)$ & $.44(.31)$ & .03 \\
\hline Correct Right Sequence & $.14(.21)$ & $.10(.16)$ & .21 & $.05(.11)$ & $.07(.13)$ & .17 \\
\hline Correct Wrong Sequence & $.07(.09)$ & $.07(.09)$ & .00 & $.08(.09)$ & $.10(.13)$ & .18 \\
\hline Correct Overall & $.61(.32)$ & $.64(.31)$ & .10 & $.58(.26)$ & $.60(.25)$ & .08 \\
\hline Wrong Word & $.04(.05)$ & $.05(.11)$ & .12 & $.06(.09)$ & $.07(.11)$ & .10 \\
\hline
\end{tabular}

Note. Standard deviations are in parentheses. All performance scores are denoted in proportion.

$d=$ Cohen's $d$ effect size calculation for between subjects.

Letter Series. The number of correct solutions was submitted to a 2 (session) $\times$ 2 (group) mixed model ANOVA. There was a main effect of session, $F(1,46)=6.35$, $p=.02, \eta^{2}=.12$. Specifically, collapsed across group, there were significantly more correct solutions at posttest $(M=11.46, S D=4.38)$ relative to pretest $(M=10.44$, $S D=4.18$ ). Importantly, the critical session $\times$ group interaction was not significant, $F(1,46)=2.88, \quad p=.10, \eta^{2}=.06$, indicating no far transfer to reasoning (see Table 15 ). There was also no effect of group, $p=.63$. 


\section{PLASTICITY OF THREE SUB-FUNCTIONS OF INHIBITION}

Table 15

Pretest - Posttest Performance in Letter Series and Digit Symbol Separately for Training and No Training Control

\begin{tabular}{|c|c|c|c|c|c|c|}
\hline \multirow[b]{2}{*}{ Task } & \multicolumn{3}{|l|}{$\begin{array}{l}\text { Training } \\
(n=24)\end{array}$} & \multicolumn{3}{|c|}{$\begin{array}{l}\text { No Training Control } \\
(n=24)\end{array}$} \\
\hline & Pre & Post & $d$ & Pre & Post & $d$ \\
\hline Letter Series & $10.50(4.28)$ & $10.83(4.68)$ & .07 & $10.37(4.17)$ & $12.08(4.06)$ & .42 \\
\hline Digit Symbol & $61.46(15.37)$ & 65.42 (15.65) & .26 & 62.25 (14.79) & $65.46(17.77)$ & .20 \\
\hline
\end{tabular}

Note. Standard deviations are in parentheses. Letter Series and Digit Symbol were scored by the average number of correct solutions.

$d=$ Cohen's $d$ effect size calculation for between subjects.

Digit Symbol. The number of correct solutions was submitted to a 2 (session) $\times$ 2 (group) mixed model ANOVA. There was a main effect of session, $F(1,46)=15.97$, $p<.001, \eta^{2}=.26$. Collapsed across group, performance was shown to be significantly better at posttest $(M=65.44, S D=16.56)$ relative to pretest $(M=61.85, S D=14.93)$. No other effects were significant, $p s>.67$ (see Table 15).

\section{Discussion}

There were two main purposes of this study: (a) examine the retest practice effects of three sub-functions of inhibition training in older adults, and (b) explore the hierarchical pattern of transfer effects (near-near, near, and far) associated with a multitask approach to inhibition training among older adults.

\section{Reliabilities}

One factor that could impact the ability to see training and transfer effects is the reliabilities of the tasks used. Therefore, test-retest reliabilities were calculated using Cronbach's alpha separately for pretest-posttest, as well as the six practice sessions 


\section{PLASTICITY OF THREE SUB-FUNCTIONS OF INHIBITION}

collapsed across training and control groups. Overall, the reliabilities of all the training tasks were consistently high $(\alpha>.78)$ and most outcome measures also showed a satisfactory reliability $(\alpha>.59$ for all measures except for the multiple-choice response index of the Reading with Distraction, 0-back and 1-back conditions of the N-Back task, as well as the RT variables of the Directed Forgetting task, where the $\alpha$ ranged from -.16 to .46). This suggests that caution needs to be taken in interpreting the transfer effects to those specific outcome measures. 


\section{PLASTICITY OF THREE SUB-FUNCTIONS OF INHIBITION}

Table 16

Test-retest Reliabilities using Cronbach's Alpha - Collapsed across Training and Control Groups

\begin{tabular}{|c|c|c|}
\hline Task & Pretest-posttest & Six Sessions \\
\hline \multicolumn{3}{|l|}{ Local-Global (RT) } \\
\hline Congruent - Local & .842 & .929 \\
\hline Congruent - Global & .935 & .902 \\
\hline Incongruent - Local & .773 & .928 \\
\hline Incongruent - Global & $.932^{\S}$ & .903 \\
\hline Neutral - Local & .708 & .932 \\
\hline Neutral - Global & .928 & .902 \\
\hline \multicolumn{3}{|l|}{ Reading with Distraction } \\
\hline Low distraction (Reading speed) & .911 & $\mathrm{n} / \mathrm{a}$ \\
\hline High distraction (Reading speed) & .955 & $\mathrm{n} / \mathrm{a}$ \\
\hline Multiple-choice (Distractor response) & .410 & $\mathrm{n} / \mathrm{a}$ \\
\hline \multicolumn{3}{|l|}{ N-Back (accuracy) } \\
\hline 0-Back & $.215^{\S}$ & $\mathrm{n} / \mathrm{a}$ \\
\hline 1-Back & .463 & .854 \\
\hline 2-Back & .637 & .902 \\
\hline 3-Back & $.615^{\S}$ & .784 \\
\hline \multicolumn{3}{|l|}{ Directed Forgetting } \\
\hline TBF (RT) & $-.163^{\omega}$ & $\mathrm{n} / \mathrm{a}$ \\
\hline New (RT) & .460 & $\mathrm{n} / \mathrm{a}$ \\
\hline TBR (Hit rate) & .669 & $\mathrm{n} / \mathrm{a}$ \\
\hline TBF (Hit rate) & .590 & $\mathrm{n} / \mathrm{a}$ \\
\hline \multicolumn{3}{|l|}{ Go - No Go } \\
\hline False Alarms (full sample) & .810 & .916 \\
\hline False Alarms (reduced sample) & $.808^{8}$ & $.893^{\circ}$ \\
\hline \multicolumn{3}{|l|}{ Stroop (RT) } \\
\hline Congruent & .933 & $\mathrm{n} / \mathrm{a}$ \\
\hline Incongruent & .876 & $\mathrm{n} / \mathrm{a}$ \\
\hline Neutral & .945 & $\mathrm{n} / \mathrm{a}$ \\
\hline
\end{tabular}

Note. $\mathrm{n} / \mathrm{a}=$ not applicable; $\mathrm{TBF}=$ to-be-forgotten; TBR = to-be-remembered.

$\S_{n=47 ;}{ }^{\gamma} n=35 ;{ }^{\omega} n=23 ;{ }^{\circ} n=14$. 


\section{PLASTICITY OF THREE SUB-FUNCTIONS OF INHIBITION}

\section{Retest Practice Effects}

The results of this study suggest that older adults are able to improve performance on tasks measuring three sub-functions of inhibition, including Local-Global (access), N-Back (deletion), and Go-No Go (restraint).

Local-Global. An examination of session 1 only (prior to any practice effects) revealed an absence of the global precedence effect in this older adult sample. This is in line with previous research, which shows that while young adults display a global processing bias older adults do not (e.g., Navon, 1977; Thomas et al., 2007). Importantly, the findings also indicate that older adults are able to reduce interference from to be ignored stimulus dimensions with practice. Furthermore, the improvement to the access sub-function of inhibition appears to be more pronounced when the disruption comes from the local features of a stimulus rather than from the global features. This suggests that the local dimension might be by default at older adults' focus of attention and thus is disruptive when trying to process global information. This supports a local precedence effect in older adults (e.g., Slavin et al., 2002, also see Chapter 2).

Using a Local-Global paradigm, Slavin et al. (2002) showed a local precedence in healthy older adults, as well as in individuals suffering from probable or possible Alzheimer's disease. Given that healthy older adults do not always show a local precedence - i.e., sometimes they demonstrate a lack of global precedence (Slavin et al., 2002; Thomas et al., 2007), future research should explore the association between a narrowing of visual attention, changes to underlying brain mechanisms, and cognitive risk factors for probable or possible Alzheimer's disease in older adults. Important in this context, the current study shows that distraction from local dimensions of a stimulus can 


\section{PLASTICITY OF THREE SUB-FUNCTIONS OF INHIBITION}

be improved upon in older adults. This finding may have implications for protection against cognitive decline in later years, but future research would first need to explore this possibility.

Additionally, these findings have implications for driving safety in an older adult population. In particular, road safety programs geared towards older adults could implement training programs aimed at reducing local biases in focused attention. Evidence gathered from multiple research studies - using a meta-analytic approach demonstrated the dangers of narrowing visual attention during driving (Clay et al., 2005). The results from this study offer support for the success of this type of training approach.

N-Back. For the N-Back task, the change in overall accuracy across six retest practice sessions was examined within the 1-Back, 2-Back and 3-Back conditions. As expected, given previous research, performance worsened as demands on deletion increased (i.e., 1-Back > 2-Back $>$ 3-Back) - an effect that is exacerbated with age (Dobbs \& Rule, 1989; Kirchner, 1958; Verhaeghen \& Basak, 2005). Although all three conditions showed improved accuracy across sessions, the most challenging 2-Back and 3-Back conditions demonstrated the biggest benefits. This suggests that, with practice, older adults become more efficient at removing no longer relevant information from their working memory, thereby making more room to store and process task relevant information.

Go-No Go. Using the Go-No Go task, older adults demonstrated reductions in the production of false alarms across six retest practice sessions. This suggests that older adults are able to improve upon their ability to withhold an automatic motoric response that is inappropriate for the task at hand, indexing better restraint. This pattern of 


\section{PLASTICITY OF THREE SUB-FUNCTIONS OF INHIBITION}

enhanced performance was maintained after removing subjects with high miss rates (i.e., > .30). The malleability of the restraint sub-function of inhibition is in line with previous work, which shows that with practice older adults are able to increase performance by better withholding an automatic word reading response - as measured by the Stroop task (i.e., Wilkinson \& Yang, 2012). Taken together, these studies show that older adults can learn to withhold automatic responses (motoric or cognitive) that are inappropriate for the task at hand.

In sum, the current retest practice effect data demonstrate plasticity of three distinct sub-functions of inhibition: access, deletion, and restraint in older adults.

\section{Transfer Effects}

The data presented here show evidence in support of near-near transfer effects following three sub-functions of inhibition training. Although there is also some evidence suggesting near transfer to the Stroop task, overall, near and far transfer following three sub-functions of inhibition training is not supported.

Near-near transfer. Near-near transfer was demonstrated in all three subfunctions of inhibition among older adults. This is in line with previous research using executive function training (e.g., Bherer et al., 2005; Karbach \& Kray, 2009). Specifically, near-near transfer is evaluated by tasks that are parallel versions with the ones used during training - except the items are typically varied. In the current study, the digit versions of Local-Global, N-Back and Go-No Go were used to assess near-near transfer (letter versions of the same tasks were used during training). The findings from each task will be described below. 


\section{PLASTICITY OF THREE SUB-FUNCTIONS OF INHIBITION}

Local-Global. The data from the Local-Global task at pretest and posttest sessions revealed that, overall, responding to the local dimension was faster than to the global dimension. This supports a local precedence effect. Taken together with the absence of a global precedence effect revealed at session 1 of practice, these data strongly support the absence of a global precedence effect with increasing age and even suggest a local precedence effect, which is in line with previous research (e.g., Slavin et al., 2002; Thomas et al., 2007). Also, near-near transfer to the digit Local-Global task in older adults following training was shown. Additional follow-up analyses highlighted training related benefits in older adults' ability to keep irrelevant information from entering their focus of attention tended to be specific to local information during global processing. This is in line with the patterns seen in the retest practice effect data. Although interesting, the specificity of transfer during global dimension focus must be interpreted with caution, given the lack of a significant group by session interaction. Regardless, reduction in interference scores at posttest was only evident in the training group, and this supports near-near transfer to the digit Local-Global task in older adults.

$\boldsymbol{N}$-Back. Near-near transfer effects were also revealed with the N-Back task, as a measure of the deletion sub-function of inhibition. In those who received three subfunctions of inhibition training, pretest-posttest improvement in accuracy was seen specifically for condition that most require efficient deletion: 2-Back and 3-Back. These two conditions are the most cognitively demanding considering they require keeping multiple trials in working memory (the current one, plus the two or three previously presented trials), while simultaneously needing manipulate this information by removing pieces that are no longer relevant. Our findings show that training inhibition leads to 


\section{PLASTICITY OF THREE SUB-FUNCTIONS OF INHIBITION}

improvements in the ability to delete no longer relevant information from working memory - as measured by a digit N-Back task that is structurally equivalent to the letter N-Back task used during training.

Go-No Go. Last, the current study also demonstrated near-near transfer effects to a task that assesses the restraint sub-function of inhibition. This shows that following inhibition training, older adults are better able to withhold automatic responses that are task inappropriate - as measured by a Go-No Go task similar to the one used at training, but with digit (instead of letter) stimuli.

In sum, the results suggest clear near-near transfer effects in older adults following three sub-functions of inhibition training, with solid effects for all three subfunctions: access, deletion and restraint. These results are in line with previous work, which demonstrates that older adults maintain the capacity to transfer trained skills to structurally similar tasks that use varying items (e.g., Bherer et al., 2005; Karbach \& Kray, 2009). This indicates that structural similarities between the training and transfer tasks are important for eliciting transfer effects in older adults. Consistent with a previous study showing non-item-specific retest learning in older adults (Yang et al., 2009a), the current findings suggest that the inhibition training benefits are not item specific, but occur at a more conceptual level and can be generalized to stimuli other than those used during the training. These findings are also in line with the finding of the ACTIVE project as described in Ball et al. (2002), where it was demonstrated that transfer was most reliably shown in the target domain that was trained. In this study separate groups of older adults received training in either memory, reasoning or processing speed. The results demonstrated that the posttest improvements were limited to the trained domain 


\section{PLASTICITY OF THREE SUB-FUNCTIONS OF INHIBITION}

(e.g., those that received memory training showed posttest improvements in memory, but not in reasoning or speed).

Near transfer. The current study demonstrates limited near transfer effects of three sub-functions of inhibition training in older adults, which is in line with previous research (Dahlin et al., 2008a; Dahlin et al., 2008b). Specifically, near transfer is evaluated with outcome measures that tap into the same cognitive functions as the training task but using structurally distinct tasks (e.g., Willis et al., 1981). In the current study, Reading with Distraction was used as the near transfer task for the access subfunction of inhibition (trained with Local-Global), Directed Forgetting for deletion (trained with N-Back) and the Stroop task for restraint (trained with Go-No Go). Although the omnibus analyses did not reveal near transfer to any of the tasks mentioned above among older adults, visual inspection and a follow-up test demonstrated that only the training group, but not controls, tended to show improved performance on the Stroop task following training.

For the access and deletion sub-functions of inhibition, the Local-Global and N-Back tasks were implemented at training and the Reading with Distraction and Directed Forgetting tasks were used to assess near transfer effects, respectively. Even though the training and transfer tasks share overlapping cognitive domains (Hasher et al., 1999; Hasher et al., 2007), the current study provides no evidence of near transfer. According to Dahlin et al. (2008b), a lack of near transfer may result from age-related changes to task specific brain regions. Although the current study did not measure brain activation patterns, previous research suggests that brain regions involved in tasks that utilize the access or deletion sub-functions of inhibition (including the lingual gyrus and 


\section{PLASTICITY OF THREE SUB-FUNCTIONS OF INHIBITION}

parahippocampal regions for access tasks [see Fink et al., 1997; Gazzaley et al., 2005], and various frontal and parietal regions during deletion tasks [see Owen et al., 2005; Wylie et al., 2008]) undergo substantial age-related changes (e.g., Dempster, 1992; Raz, 2000; Hedden \& Gabrieli, 2004; West, 1996). This may have limited the ability of older adults to transfer the training benefits to a structurally different task measuring the same cognitive ability (Dahlin et al., 2008a; Dahlin et al., 2008b).

Another possible explanation for the limited near transfer lies in the differences in the type and quality of the stimuli used during training as compared to the pretest and posttest sessions. Specifically, individually presented number stimuli were presented at pretest and posttest for the Local-Global and N-Back tasks, while word stimuli were used in the Reading with Distraction and Directed Forgetting tasks. In this way, differences in basic task features (number vs. letter), as well as task processing demands (single number vs. semantic word processing) between the training and transfer tasks may account for the lack of near transfer effects in older adults.

Last, for the restraint sub-function of inhibition, the Go-No Go task was used at training and the Stroop task was used to assess transfer. These tasks are similar in that they both require participants to withhold automatic urges - although one is a motor response (i.e., withhold a key press in the Go-No Go task), while the other is cognitive (i.e., withhold a reading response in the Stroop task). The follow-up test, in combination with Cohen's $d$ values, suggest that the training group has a larger pretest-posttest improvement than the control group, indicating promising near transfer of inhibition training to the Stroop task. However, this conclusion should be interpreted with caution given the lack of session by group interaction. 


\section{PLASTICITY OF THREE SUB-FUNCTIONS OF INHIBITION}

Several speculations are offered to explain this trending transfer effect to the Stroop task. First, in both the Stroop and Go-No Go task, the stimuli are presented one at a time, thereby requiring that participants make item-by-item responses. The other near transfer tasks involved a sizable amount of information to process - i.e., a whole passage or a series words, which requires higher-level conceptual integration. This distinction in cognitive complexity may explain why there was trending near transfer patterns to the Stroop task, but not to Reading with Distraction or Directed Forgetting. Second, it is possible to conceive of the Stroop task as requiring various types of inhibition, including withholding an automatic word reading response (restraint), as well as keeping irrelevant word information outside one's focus of attention (access). Given that the Stroop task potentially incorporates two types of inhibition, it may have greatly benefited from a multi-task approach to inhibition training.

Furthermore, Dahlin et al. (2008b) explained their lack of near transfer in older adults using a neuroimaging framework, whereby age-related changes in task-associated brain regions limit the generalizability of training task improvement to other tasks that were not trained. Future research should endeavour to collect brain imaging data to ensure overlap between the near transfer tasks and the training tasks. In addition, a young adult sample should also be tested to inform the hypothesis that age-related brain changes are limiting near transfer in three sub-function of inhibition training among older adults.

In sum, near transfer effects were limited in older adults following three subfunctions of inhibition training. Training gains do not easily generalize to alternative untrained tasks, such as Reading with Distraction and Directed Forgetting, believed to tap into the same sub-functions of inhibition as the training tasks; however, some promising 


\section{PLASTICITY OF THREE SUB-FUNCTIONS OF INHIBITION}

evidence suggests near transfer to the Stroop task. I offer several interpretations for this discrepancy, including basic task features and processing requirements. Taken together, these findings suggest that to elicit near transfer in older adults, the structural overlap between the training and the transfer tasks may be just as important as the cognitive overlap.

Far transfer. The absence of far transfer-effects in older adults following executive function training is in line with previous studies (e.g., Dahlin et al., 2008a; Li et al., 2008). In general, far transfer is assessed when the training and transfer tasks do not overlap in cognitive ability or task structure (Karbach \& Kray, 2009). In the current study, generalizability of the training tasks to visuospatial working memory (Corsi Block), episodic memory (Word List Recall), reasoning (Letter Series), and processing speed (Digit Symbol) was evaluated; however, far transfer effects were not elicited in any of the above-mentioned cognitive domains.

Training-related improvements in the three sub-functions of inhibition do not lead to performance gains in cognitively distant domains, such as working memory, episodic memory, reasoning, and processing speed. This finding can be interpreted in two ways: (a) the association between these cognitive domains and inhibition is limited, or (b) far transfer effects are hard to elicit in older adults. First, the association between working memory and inhibition, as outlined by the inhibitory deficit hypothesis of aging (Hasher et al., 1999; Hasher et al., 2007), suggests that inhibitory processes allow one to control and manipulate the contents of working memory. Enhanced inhibition should thus result in a more efficient use of the working memory storage space by de-cluttering or minimizing the presence of task-irrelevant information, which improves efficiency and 


\section{PLASTICITY OF THREE SUB-FUNCTIONS OF INHIBITION}

expands capacity (Hasher et al., 1999; Hasher et al., 2007). However, the current study suggests that the link between inhibitory efficiency and working memory capacity does not easily translate from theory to practice. While the inhibitory deficit hypothesis of aging stipulates that de-cluttering working memory storage space increases the speed and accuracy with which one can retrieve information from memory, the current results do not support this. For example, in the episodic memory task used in the current study, Word List Recall, the pretest to posttest improvement was equivalent for the training and control group. However, these effects may be task specific and may not hold if other tasks assessing working memory and episodic memory were administered.

Second, far transfer may be hard to elicit in older adults. This may be due to the extent of overlap in task-relevant brain regions between the transfer and training tasks and/or age-related changes in these brain regions (Dahlin et al., 2008b). Perhaps the various frontal brain regions involved in inhibition and memory tasks (e.g., Casey et al., 1997; Chikazoe et al., 2009; Gazzaley et al., 2005; Milham et al., 2002; Mottaghy et al., 2000; Owen et al., 2005; Rubia et al., 2001; Wylie et al., 2008) do not overlap substantially enough for transfer effects to be seen. Furthermore, brain related changes to frontal regions, including a reduction in size and synaptic density, as well as the mechanisms that control dopamine regulation (e.g., Dempster, 1992; Erixon-Lindroth et al., 2005; Raz, 2000; Salat et al., 1999; West, 1996), may have further limited the possibility of far transfer effects in older adults (Dahlin et al., 2008b). Additional considerations should be given to the design of the training program. For example, previous work that has successfully demonstrated far transfer to older adults used a training program that continuously adapted task difficulty based on the participant's 


\section{PLASTICITY OF THREE SUB-FUNCTIONS OF INHIBITION}

individual level of performance (Brehmer et al., 2012; Mahncke et al., 2006; Smith et al., 2009; van Muijden, Band, \& Hommel, 2012). Therefore, training programs that are perpetually challenging may keep engagement levels high and thus promote far transfer.

\section{Limitations}

Although the current study makes strong contributions to the cognitive training literature, one limitation warrants mention. The current study did not collect neuroimaging data. This limits our ability to interpret the lack of near and far transfer effects in the context of the amount of overlap between the training and transfer tasks, as well as any age-related changes in the brain structures involved in the completion of various cognitive tasks. Although information about which brain areas are active during completion of the tasks used in this study can be taken from literature, additional value is garnered when assessing activated brain regions across various tasks within the same set of individuals. Future research should endeavor to do this - especially among the six cognitive tasks that target the three sub-functions of inhibition. Furthermore, brainimaging data on these tasks in both a young and older adult sample would provide further insight into the transfer effect data. For example, if older adults - as compared to young showed reduced activation in task-related brain regions, which was also associated with dampened training gains, and an absence of near and/or far transfer effects, this would support Dahlin et al.’s (2008b) hypothesis that transfer is limited in older adults due to changes in task related brain regions.

\section{Implications}

The current study demonstrates that all three sub-functions of inhibition: access, deletion, and restraint are modifiable in older adults; however, the transfer data are 


\section{PLASTICITY OF THREE SUB-FUNCTIONS OF INHIBITION}

limited mainly to near-near transfer - although there is some suggestive support for near transfer as well. Given that the goal of training studies is to generalize gains to tasks other than those used during the retest practice, the limited near and lack of far transfer in older adults found in this study - and commonly found in many other studies (e.g., Dahlin et al., 2008a; Dahlin et al., 2008b; Li et al., 2008) - urges researchers to investigate new approaches to cognitive training geared towards an aging population. Innovative approaches to training may need to focus more on changing thinking patterns as well as use more real-world materials, and focus less on changing specific cognitive abilities using lab designed computer programs.

A recent pilot study, using a unique real-world approach to cognitive training, investigated the generalizability of gist reasoning training in older adults and showed promising results (Anand et al., 2011). In general, gist training refers to the ability to utilize small bits of information and abstract it to inform general/global meaning. The training utilized real-world materials, such as books and newspaper articles, and participants were encouraged to use the strategies in their everyday life. This 'gist' thinking strategy is believed to incorporate many executive functions, including selective attention and inhibition. Pretest to posttest improvements were seen in untrained measures, such as Trail Making Test Part B (executive control), Similarities (concept abstraction) and Controlled Oral Word Association Test (verbal fluency). Although no control group was included in this pilot study, the results are very promising.

Considering the general findings of limited near and far transfer in older adults overall (also see Dahlin et al., 2008a; Dahlin et al., 2008b; Li et al., 2008), novel approaches to enhancing cognition in this population need to be explored. Additional 


\section{PLASTICITY OF THREE SUB-FUNCTIONS OF INHIBITION}

suggestions are made in the General Conclusions section. Alternatively, if sticking to a more lab-based computerized approach, matching training and transfer tasks structurally and conceptually as closely as possible is encouraged to maximize transfer in older adults. Furthermore, including programs that adapt difficulty levels to each individual participant's performance level from session to session may also be beneficial to promote the transfer effects. 


\section{GENERAL CONCLUSIONS}

\section{General Conclusions}

This dissertation adds to the current literature by demonstrating several novel findings. First, older adults are able to maintain single-task inhibition training gains for one and three years, and show durability above baseline levels at the 1-year follow-up session and trending effects three years after training. These findings offer promising evidence for the durability of executive function training benefits in older adults across a long time-interval, and extend the literature to include maintenance of inhibition.

Furthermore, age differences in all three sub-functions of inhibition were established, with performance differentially favouring young over older adults. Importantly, however, it was shown that age-related deficits in access, deletion, and restraint, when tested with the same group of individuals, are not consistently represented across various tasks. Researchers should pay careful attention when selecting tasks to evaluate age differences in inhibition as some tasks do not show reliable deficits. Additional consideration should be given to small changes in task design that may uncover age differences that would otherwise have been masked - for example, using a tagging procedure in the Directed Forgetting task (Thompson et al., 2011) or extending the duration of stimuli presentation in the Go-No Go task.

Last, novel contributions were made to the cognitive training area of research by extending the literature and demonstrating that older adults show plasticity in all three sub-functions of inhibition. This has implications for the design of future training studies geared towards older adults - e.g., to help improve functioning in everyday activities of daily living, such as driving. Furthermore, it was shown that after training multiple subfunctions of inhibition, older adults demonstrate clear transfer to tasks that are 


\section{GENERAL CONCLUSIONS}

structurally and cognitively identical to those used at training. This shows that the training effects in inhibition are not item specific and can be transferred to a different set of stimuli; however, the general lack of near and far transfer adds evidence against the generalizability of lab-based approaches to untrained cognitive or everyday functions in older adults. Although the current dissertation cannot speak to whether or not age-related brain changes are underlying the limited generalizability of training to transfer tasks in older adults, this is a plausible theory (Dahlin et al., 2008b).

Future research investigating cognitive training in older adults needs to explore other avenues in the pursuit of transfer effects - e.g., mindfulness meditation (mental exercise that requires one to focus on the sensations of the body and breathing, while keeping the mind free from distraction). In young adults, following brief mindfulness training (four 20-minute sessions), cognitive benefits were demonstrated in attention, and executive functions (including inhibition) using a variety of different tasks - e.g., N-Back (Zeidan, Johnson, Diamond, David, \& Goolkasian, 2010). To date, no research has evaluated the benefits of a structured mindfulness-based training program on cognitive outcomes in older adults. Mindfulness meditation may be an alternative approach to training that could globally benefit an older adult sample and should be explored further. In addition, aerobic training has been demonstrated to show promising cognitive benefits in older adults (for a meta-analytic review see Colcombe \& Kramer, 2003). Ideally, an efficient and valuable training program would be one that shows benefits that extend beyond the tasks used at training. Using approaches, such as mindfulness and aerobic exercise training, that affect underlying brain mechanisms - e.g., through increased 


\section{GENERAL CONCLUSIONS}

cerebral blood flow - may offer more generalized cognitive benefits in older adults and warrants further investigation to refine training techniques and optimize outcomes. 


\section{Appendix A}

\section{REB Approval Form for Wilkinson and Yang (2012; Chapter 1)}

\section{RYERSONUNIVERSITY RESEARCH ETHICS BOARD}

To: Andrea Wilkinson

Psychology

Re: REB 2008-195: Cognitive Plasticity of Inhibition among Older Adults

Date: September 23, 2008

Dear Andrea Wilkinson,

The review of your protocol REB File REB 2008-195 is now complete. The project has been approved for a one year period. Please note that before proceeding with your project, compliance with other required University approvals/certifications, institutional requirements, or governmental authorizations may be required.

This approval may be extended after one year upon request. Please be advised that if the project is not renewed, approval will expire and no more research involving humans may take place. If this is a funded project, access to research funds may also be affected.

Please note that REB approval policies require that you adhere strictly to the protocol as last reviewed by the REB and that any modifications must be approved by the Board before they can be implemented. Adverse or unexpected events must be reported to the REB as soon as possible with an indication from the Principal Investigator as to how, in the view of the Principal Investigator, these events affect the continuation of the protocol.

Finally, if research subjects are in the care of a health facility, at a school, or other institution or community organization, it is the responsibility of the Principal Investigator to ensure that the ethical guidelines and approvals of those facilities or institutions are obtained and filed with the REB prior to the initiation of any research.

Please quote your REB file number (REB 2008-195) on future correspondence.

Congratulations and best of luck in conducting your research.

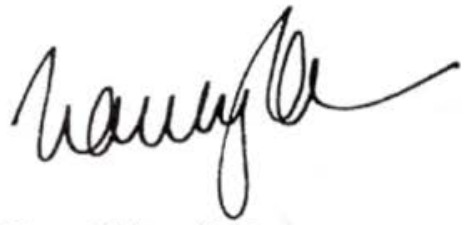

Nancy Walton, Ph.D.

Chair, Research Ethics Board 


\title{
Appendix B
}

\section{REB Approval Form for the 'Age Differences in Three Sub-functions of Inhibition'}

\author{
Study (Chapter 2)
}

\section{RYERSONUNIVERSITY}

To: Andrea Wilkinson

Psychology

Re: REB 2012-098: Breaking Down Inhibition: Validating a Three-component Structure of Inhibition

Date: May 2, 2012

Dear Andrea Wilkinson,

The review of your protocol REB File REB 2012-098 is now complete. The project has been approved for a one year period. Please note that before proceeding with your project, compliance with other required University approvals/certifications, institutional requirements, or governmental authorizations may be required.

This approval may be extended after one year upon request. Please be advised that if the project is not renewed, approval will expire and no more research involving humans may take place. If this is a funded project, access to research funds may also be affected.

Please note that REB approval policies require that you adhere strictly to the protocol as last reviewed by the REB and that any modifications must be approved by the Board before they can be implemented. Adverse or unexpected events must be reported to the REB as soon as possible with an indication from the Principal Investigator as to how, in the view of the Principal Investigator, these events affect the continuation of the protocol.

Finally, if research subjects are in the care of a health facility, at a school, or other institution or community organization, it is the responsibility of the Principal Investigator to ensure that the ethical guidelines and approvals of those facilities or institutions are obtained and filed with the REB prior to the initiation of any research.

Please quote your REB file number (REB 2012-098) on future correspondence.

Congratulations and best of luck in conducting your research.

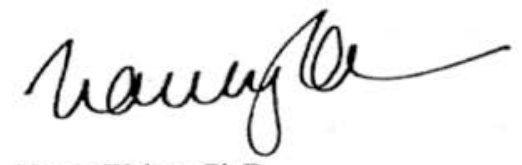

Nancy Walton, Ph.D.

Chair, Research Ethics Board 
APPENDICES

\section{Appendix C}

REB Approval Form for the 'Plasticity of Three Sub-functions of Inhibition in Older

Adults’ Study (Chapter 3)

\section{RYERSONUNIVERSITY \\ RESEARCH ETHICS BOARD}

To: Andrea Wilkinson

Psychology

Re: REB 2010-313: Plasticity of inhibition: Retest practice and transfer effect of 3 inhibitory sub-type training

Date: February 4, 2011

\section{Dear Andrea Wilkinson,}

The review of your protocol REB File REB 2010-313 is now complete. The project has been approved for a one year period. Please note that before proceeding with your project, compliance with other required University approvals/certifications, institutional requirements, or governmental authorizations may be required.

This approval may be extended after one year upon request. Please be advised that if the project is not renewed, approval will expire and no more research involving humans may take place. If this is a funded project, access to research funds may also be affected.

Please note that REB approval policies require that you adhere strictly to the protocol as last reviewed by the REB and that any modifications must be approved by the Board before they can be implemented. Adverse or unexpected events must be reported to the REB as soon as possible with an indication from the Principal Investigator as to how, in the view of the Principal Investigator, these events affect the continuation of the protocol.

Finally, if research subjects are in the care of a health facility, at a school, or other institution or community organization, it is the responsibility of the Principal Investigator to ensure that the ethical guidelines and approvals of those facilities or institutions are obtained and filed with the REB prior to the initiation of any research.

Please quote your REB file number (REB 2010-313) on future correspondence.

Congratulations and best of luck in conducting your research.

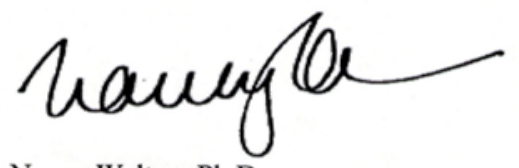

Nancy Walton, Ph.D.

Chair, Research Ethics Board 
Appendix D

Consent Agreement for the 1-year Follow-up Sample (Chapter 1)

\author{
Ryerson University Consent Agreement \\ Cognitive Plasticity of Inhibition among Older Adults: 1-year follow-up \\ PhD Student: Andrea Wilkinson \\ Supervising Faculty Member: Dr. Lixia Yang \\ Department of Psychology, Ryerson University \\ Email: a5wilkin@psych.ryerson.ca \\ Phone Number: (416)979-5000 ext. 4987
}

You are being asked to participate in a research study. Before you give your consent to participate, please read the following information. If you have any questions, please feel free to ask.

Investigators: Andrea Wilkinson (principal investigator, M.A. Student) \& Lixia Yang (supervising investigator, Ph.D.), Department of Psychology, Ryerson University.

Purpose of the Study: The purpose of the original study was to train elderly people to efficiently focus their attention on relevant information and inhibit irrelevant information. The purpose of the 1-year follow-up session is to evaluate the long term maintenance of the training effects.

Description of the Study: The study will take place in the Cognitive Aging Lab (SBB 240) of Ryerson University located at 105 Bond St., Toronto. The followup session will consist of one approximately $1-1.5$ hour session. At this session you will be asked to complete one Stroop task training session (approximately 30 minutes), and some computerized and paper-pencil cognitive tasks to evaluate the long term maintenance of the training effects. You may refuse to participate in any of the tasks at any point without any negative consequences.

Risks and Benefits: Study risk is minimal. The risk, if any, will be no greater than that encountered in everyday life. The stimuli used in this study are commonly encountered in books, newspapers, radio, TV, or movies. Every effort will be taken to ensure that you are as comfortable as possible throughout this training program; however, you may withdraw from the study at any time without any penalty. The major benefit from participating in this study is the opportunity to learn and practice skills in inhibitory processing. You will also contribute to the process of scientific research.

Confidentiality: We will take great care to ensure protection of your confidentiality and privacy. Any information learned about you during this study will be kept confidential, and neither your name nor any other identifying information will be made available to anyone other than the investigators. This 


\section{APPENDICES}

information will be secured in a locked filing cabinet at all times. All physical copies of the documents and data will be stored in a secure area and will be shredded when no longer required. In any reports, publications or presentation, no individual will be identified, and only group results will be presented.

Voluntary Nature of Participation: Participation in this study is voluntary. Your choice of whether or not to participate has no bearing on your past, present or future relationship with Ryerson University. If you decide to participate, you are free to withdraw your participation at any time before completion. Since the data will be coded, participants may withdraw up until the time of data analysis. Moreover, you may refuse to answer particular questions, or part of any questionnaires without any penalty.

Compensation: You will be compensated \$10/hour for participation in this follow-up session. Compensation will be provided fully to all participants, even those who are not able to complete the session.

Questions about the Study: If you have any questions about the research now, please ask. If you have questions about the research later, you may contact Andrea Wilkinson at (416) 979-5000 (ext.4987). If you have questions regarding your rights as a human subject and participant in this study, you may contact the Ryerson University Research Ethics Board for information.

Research Ethics Board
c/o Office of the Associate Vice President, Academic
Ryerson University
350 Victoria Street
Toronto, Ontario M5B 2K3

(416) $979-5042$

Agreement: Your signature below indicates that you have read the information in this agreement and have had the chance to ask any questions you have about the study. Your signature also indicates that you agree to be in the study and have been told that you can change your mind and withdraw your consent to participate at any time.

By signing below, I acknowledge that I have read and understood all of the above statements and agree to voluntarily participate in this study.

Name of Participant (please print)

Signature of Participant

Date

Signature of Investigator

Date 


\title{
APPENDICES
}

\section{Appendix E}

Consent Agreement for the 3-year Follow-up Sample (Chapter 1)

\author{
Ryerson University Consent Agreement \\ Cognitive Plasticity of Inhibition in Older Adults: 3-year Follow-up \\ PhD Student: Andrea Wilkinson \\ Supervising Faculty Member: Dr. Lixia Yang \\ Department of Psychology, Ryerson University \\ Email: a5wilkin@psych.ryerson.ca \\ Phone Number: (416)979-5000 ext. 4987
}

You are being asked to participate in a research study. Before you give your consent to participate, please read the following information. If you have any questions, please feel free to ask.

Investigators: Andrea Wilkinson (principal investigator, Ph.D. Student) \& Lixia Yang (supervising investigator, Ph.D.), Department of Psychology, Ryerson University.

Purpose of the Study: The purpose of the original study was to train older adults to efficiently focus their attention on relevant information and inhibit irrelevant information. The purpose of the 3-year follow-up session is to evaluate the long term maintenance of the training effects.

Description of the Study: The study will take place in the Cognitive Aging Lab (SBB 240) of Ryerson University located at 105 Bond St., Toronto. The followup session will consist of one session. At this session you will be asked to complete one Stroop task training session (approximately 15 minutes).You may discontinue participation at any point without any negative consequences.

Risks and Benefits: Study risk is minimal. The risk, if any, will be no greater than that encountered in everyday life. The stimuli used in this study are commonly encountered in books, newspapers, radio, TV, or movies. Every effort will be taken to ensure that you are as comfortable as possible throughout this follow-up session; however, you may withdraw from the session at any time without any penalty. The major benefit from participating in this study is the opportunity to learn and practice skills in inhibitory processing. You will also contribute to the process of scientific research.

Confidentiality: We will take great care to ensure protection of your confidentiality and privacy. Any information learned about you during this study will be kept confidential, and neither your name nor any other identifying information will be made available to anyone other than the investigators. This information will be secured in a locked filing cabinet at all times. All physical copies of the documents and data will be stored in a secure area and will be 


\title{
APPENDICES
}

shredded when no longer required. In any reports, publications or presentation, no individual will be identified, and only group results will be presented.

Voluntary Nature of Participation: Participation in this study is voluntary. Your choice of whether or not to participate has no bearing on your past, present or future relationship with Ryerson University. If you decide to participate, you are free to withdraw your participation at any time before completion. Since the data will be coded, participants may withdraw up until the time of data analysis. Moreover, you may discontinue your participation at any time without any penalty.

Compensation: You will be compensated \$10/hour for participation in this follow-up session. Compensation will be provided fully to all participants, even those who are not able to complete the session.

Questions about the Study: If you have any questions about the research now, please ask. If you have questions about the research later, you may contact Andrea Wilkinson at (416) 979-5000 (ext.4987). If you have questions regarding your rights as a human subject and participant in this study, you may contact the Ryerson University Research Ethics Board for information.

\author{
Research Ethics Board \\ c/o Office of the Associate Vice President, Academic \\ Ryerson University \\ 350 Victoria Street \\ Toronto, Ontario M5B 2K3
}

(416) $979-5042$

Agreement: Your signature below indicates that you have read the information in this agreement and have had the chance to ask any questions you have about the study. Your signature also indicates that you agree to be in the study and have been told that you can change your mind and withdraw your consent to participate at any time.

By signing below, I acknowledge that I have read and understood all of the above statements and agree to voluntarily participate in this study.

Name of Participant (please print)

Signature of Participant

Date

Signature of Investigator

Date 


\section{Appendix F}

Oral Debriefing for the 1 and 3-year Follow-up (Chapter 1)

\section{One-year Follow-up}

The purpose of this 1-year follow-up was to evaluate the maintenance of the training and transfer effects one year post training. Specifically, because we found overall improvement in inhibitory processing across training sessions, we wanted to determine whether this improvement is maintained one year following training. Furthermore, we found that there was enhanced performance at posttest (relative to pretest) on certain cognitive tasks (e.g., attention, speed of processing, and reasoning), and therefore, we also wanted to determine whether these transfer effects were maintained 1-year later.

\section{Three-year Follow-up}

The purpose of this three-year follow-up is to evaluate the maintenance of the practice effects three years following initial training as well the performance maintenance evidenced one-year following training. Recall that we were studying training gains in the ability to keep irrelevant information outside our focus of attention (i.e., inhibition). Specifically, because we found overall improvements in inhibitory processing across training sessions (three years ago), we wanted to determine whether this improvement could be preserved at various time intervals (i.e., one and three years later). At the one-year followup, participants not only maintained the inhibitory performance levels from the training; but, they performed even better! Given this, we want to determine whether this robust maintenance of training gains is still shown three years later. Looking at the preservation of training gains across time will help in the development of effective programs that not only improve mental functions in the short-term; but, display lasting benefits over time. 
Appendix G

Consent Agreement for the Young Adult Sample (Chapter 2)

RYERSON

UNIVERSITY

DEPARTMENT OF PSYCHOLOGY FACULTY OF ARTS

\author{
Ryerson University Consent Agreement \\ Breaking Down Inhibition: Validating a Three-component Structure of Inhibition \\ Andrea Wilkinson \\ Department of Psychology, Ryerson University \\ Email: a5wilkin@psych.ryerson.ca \\ Phone Number: (416) 979-5000 ext. 4987
}

You are being asked to participate in a research study. Before you give your consent to participate, please read the following information. If you have any questions, please feel free to ask.

Investigators: Andrea Wilkinson (principal investigator), Ph.D. student, Psychology Department, Ryerson University and Dr. Lixia Yang (faculty supervisor), Associate Professor, Psychology Department, Ryerson University.

Purpose of the Study: The purpose of this study is to examine the composition of a mental ability named inhibition in younger and older adults. This ability is suggested to have three pieces: access - keeps unimportant information outside your focus of attention, deletion - gets rid of no longer important information, and restraint - withholds urges. The main goal of the current study is to determine whether inhibition has three separable pieces or something else. Given that research shows this mental ability tends to decline as we get older, a secondary goal of this study is to evaluate age effects on the composition of inhibition.

Description of the Study: The study will take place in the Psychology Research Training Centre of Ryerson University located at 105 Bond Street. Participation in this experiment will consist of a single, two-hour session. During the session, you will complete six computerized tasks that assess the various types of inhibition as well as several paper-and-pencil tasks that tests on a broad range of skills, including speed of processing and vocabulary. You will also be asked to complete a set of questionnaires that include queries on your demographic information (e.g., age, sex, and native language), education (i.e., the number of years in educational category), and personal health (e.g., illness, vision or hearing problems, any previous head injuries, and current medication use). We request this information in order to assess factors that affect the experimental tasks you are going to be asked to perform in this study. You may refuse to answer any 


\section{APPENDICES}

particular questions or any questionnaires - including those pertaining to your health and personal information - without any negative consequences.

Risks and Benefits: Study risk is minimal. The risk, if any, will be no greater than that encountered in everyday life. The materials used in this study are commonly encountered in books, newspapers, radio, TV, or movies. Every effort will be taken to ensure that you are as comfortable as possible throughout this experiment; however, you may withdraw from the study at any time without any penalty. The major benefit from participating in this study is the opportunity to learn and practice skills in inhibitory processing. However, we cannot guarantee any individual benefit to you as a result of participating.

When we look at all of the information we collect, sometimes some participants' scores are such that we cannot use them in the final analysis. It may happen that your information is not used in the final study; however, we will not be directly informing participants about whether or not their individual information is used.

Confidentiality: We will take great care to ensure protection of your confidentiality and privacy. Any information learned about you during this study will be kept confidential, and neither your name nor any other identifying information will be made available to anyone other than individuals involved in the research team. As additional security, each participant will receive a numerical ID. Your data will be assigned to this numerical ID to prevent this information from being linked back to you. Only the ID number will appear on electronic data files. Only individuals involved in the research team will have access to a central password-protected electronic file. All identifying information will be stored separately from this coded information and will be secured in a locked filing cabinet located in the Cognitive Aging Laboratory at Ryerson University at all times. All physical copies of the documents and data will be stored in a secure area and will be shredded when no longer required (i.e., up to 10 years). In any reports, publications or presentation, no individual will be identified, and only group results will be presented.

Voluntary Nature of Participation: Participation in this study is voluntary. Your choice of whether or not to participate has no bearing on your past, present or future relationship with Ryerson University. If you decide to participate, you are free to withdraw your participation at any time before completion of the study. You also have the option for a "walk through" session by completing some or all of the tasks, but withdrawing your data from the final data set. Moreover, you may refuse to answer particular questions, or part of any questionnaires without any penalty.

Compensation: Participants recruited from the undergraduate psychology student participant pool (i.e., SONA) will receive $2 \%$ course credit for their Introductory Psychology course. Compensation will be provided fully to all 


\section{APPENDICES}

participants, even those who do not complete the study or choose to just "walk through" the session.

Information about the Study: If you have any questions about the research now, please ask the experimenter. If you have questions or concerns about the research later, you may contact the principal investigator, Andrea Wilkinson, at (416) 979-5000 ext.4987 or a5wilkin@psych.ryerson.ca or the faculty supervisor, Dr. Lixia Yang, at (416) 979-5000 ext.6522 or lixiay@psych.ryerson.ca. If you have questions regarding your rights as a human subject and participant in this study, you may contact the Ryerson University Research Ethics Board (REB) at (416) 979-5042 for information. The REB mailing address is: Research Ethics Board, c/o Office of the Associate Vice President, Research and Innovation, Ryerson University, 350 Victoria Street, Toronto, Ontario M5B 2K3.

Agreement: Your signature below indicates that you have read the information in this agreement and have had the chance to ask any questions you have about the study. Your signature also indicates that you agree to be in the study and have been told that you can change your mind and withdraw your consent to participate at any time.

By signing below, I acknowledge that I have read and understood all of the above statements and agree to voluntarily participate in this study.

Name of Participant (please print)

Signature of Participant

Date

Signature of Investigator

Date 


\title{
APPENDICES
}

\section{Appendix H}

\author{
Debriefing Form for the Young Adult Sample (Chapter 2)
}

\section{DEBRIEFING FORM - for SONA participants}

Breaking Down Inhibition: Validating a Three-component Structure of Inhibition

Thank you very much for participating in this experiment! This document explains what we are trying to investigate.

The purpose of this study is to explore age differences in what makes up the mental ability inhibition. One group of researchers suggest that it is composed of three pieces: access - keeps unimportant information outside your focus of attention, deletion - gets rid of no longer important information and restraint - withholds urges. Research also shows that inhibition tends to decline as we get older (e.g., Hasher, Zacks, and May, 1999).

There is no current agreement on what pieces make up inhibition. Given this, the main goal of the current study is to determine whether inhibition has three separable pieces or something else, and, furthermore, to evaluate age effects on the composition of inhibition. Based on previous studies, we expect to find that inhibition has three separable pieces in younger adults (e.g., Healey et al., in press); however, with older adults, we do not have any expectations, because this is a new research question.

The independent variable will be age (younger and older), and the dependent variables will be reaction time and accuracy measures on the various computerized tasks used in the current study.

The implications of this study are in furthering our knowledge of the composition of the mental function inhibition. This will aid in the creation of effective training studies geared at improving this ability in both younger and older adults.

If you are interested in further information, you are encouraged to take a look at the references provided below. Please do not hesitate to ask the experimenter questions and/or to contact the principle investigator, Andrea Wilkinson, at 416-979-5000 (ext. 4987), if you have any questions or concerns regarding this project. Thank you for your participation!

\section{REFERENCES}

Gorfein, D. S., \& MacLeod, C.M. (Eds.). (2007). Inhibition in Cognition. Washington, DC: American Psychological Association.

Hasher, L., Zacks, R.T., \& May, C.P. (1999). Inhibitory control, circadian arousal, and age. In D. Gopher, and A. Koriat (Eds.), Attention and Performance XVII (pp. 653-675). Cambridge, MA: MIT Press.

Healey, M. K., Zacks, R. T., Hasher, L., \& Helder, E. (submitted). Inhibition and the relationship between working memory and fluid intelligence.

Nigg, J. T. (2000). On inhibition/disinhibition in development psychopathology: Views from cognitive and personality psychology and a working memory inhibition taxonomy. Psychological Bulletin, 126, 220246. 
Appendix I

Consent Agreement for the Training Group (Chapter 3)

\section{Ryerson University Consent Agreement}

Cognitive Plasticity of Inhibition

PhD Student/Principal Investigator: Andrea Wilkinson

Supervising Faculty Member: Dr. Lixia Yang

Department of Psychology, Ryerson University

Email: a5wilkin@psych.ryerson.ca; Phone Number: (416)979-5000 ext. 4987

You are being asked to participate in a research study. Before you give your consent to participate, please read the following information. If you have any questions, please feel free to ask.

Investigators: Andrea Wilkinson (principal investigator, PhD Student) \& Lixia Yang (supervising investigator, PhD), Department of Psychology, Ryerson University.

Purpose of the Study: The purpose of this project is to determine whether training or practice on cognitive tasks improves performance.

Description of the Study: The study will take place in the Cognitive Aging Lab (SBB 240) located in the Psychology Training and Research Centre on 105 Bond Street at Ryerson University. The study involves 8 sessions spread over 4 weeks: one 1.5 to 2-hour pretest session, six 1-hour practice sessions, and one 1.5 to 2hour posttest session. As such, this study can take up to 10 hours in total.

At the pretest and posttest sessions, you will take tests on a broad range of skills including those involved in the training sessions to determine the benefits of training.

Also, at the pretest session, you will complete a set of questionnaires that include queries on your demographic information (e.g., age, sex, and native language), education (i.e., the number of years at each educational institution), and personal health (e.g., illness, vision or hearing problems, any previous head injuries, and current medication use). We request this information in order to assess what factors have effects on the experimental tasks you are going to be asked to perform in this study. You may refuse to answer any particular questions or any questionnaires including those pertaining to your health and personal information, without any negative consequences.

The 6 practice sessions will be spread over 2 weeks (3 times per week). At each practice session, you will be asked to complete various computerized tasks (you will be given the opportunity to practice on each of the tasks).

Risks and Benefits: Study risk is minimal. The risk, if any, will be no greater than that encountered in everyday life. The stimuli used in this study are commonly encountered in books, newspapers, radio, TV, or movies. Every effort will be taken to ensure that you are as comfortable as possible throughout this research study; however, you may withdraw from the study at any time without 


\section{APPENDICES}

any penalty. The major benefit from participating in this study is the opportunity to learn and practice skills in mental processing. You will also contribute to the process of scientific research.

Confidentiality: We will take great care to ensure protection of your confidentiality and privacy. Any information learned about you during this study will be kept confidential, and neither your name nor any other identifying information will be made available to anyone other than the investigators. This information will be secured in a locked filing cabinet at all times. All physical copies of the documents and data will be stored in a secure area and will be shredded when no longer required. In any reports, publications or presentation, no individual will be identified, and only group results will be presented.

Voluntary Nature of Participation: Participation in this study is voluntary. Your choice of whether or not to participate has no bearing on your past, present or future relationship with Ryerson University. If you decide to participate, you are free to withdraw your participation at any time before completion. Since the data will be coded, participants may withdraw up until the time of data analysis. Moreover, you may refuse to answer particular questions, or part of any questionnaires without any penalty.

Compensation: You will be compensated \$10/hour for participation in this study. A prorated amount of compensation is available should you discontinue participation.

Questions about the Study: If you have any questions about the research now, please ask. If you have questions about the research later, you may contact Andrea Wilkinson at (416) 979-5000 (ext.4987). If you have questions regarding your rights as a human subject and participant in this study, you may contact the Ryerson University Research Ethics Board for information.

Research Ethics Board c/o Office of the Associate Vice President, Academic Ryerson University, 350 Victoria Street

Toronto, Ontario, M5B 2K3

(416) $979-5042$

Agreement: Your signature below indicates that you have read the information in this agreement and have had the chance to ask any questions you have about the study. Your signature also indicates that you agree to be in the study and have been told that you can change your mind and withdraw your consent to participate at any time.

By signing below, I acknowledge that I have read and understood all of the above statements and agree to voluntarily participate in this study.

Name of Participant (please print)

Signature of Participant

Date

Signature of Investigator

Date 
Appendix J

Consent Agreement for the Control Group (Chapter 3)

\section{Ryerson University Consent Agreement}

Cognitive Plasticity of Inhibition

PhD Student/Principal Investigator: Andrea Wilkinson

Supervising Faculty Member: Dr. Lixia Yang

Department of Psychology, Ryerson University

Email: a5wilkin@psych.ryerson.ca; Phone Number: (416)979-5000 ext. 4987

You are being asked to participate in a research study. Before you give your consent to participate, please read the following information. If you have any questions, please feel free to ask.

Investigators: Andrea Wilkinson (principal investigator, PhD Student) \& Lixia Yang (supervising investigator, PhD), Department of Psychology, Ryerson University.

Purpose of the Study: The purpose of this project is to determine whether training or practice on cognitive tasks improves performance.

Description of the Study: The study will take place in the Cognitive Aging Lab (SBB 240) located in the Psychology Training and Research Centre on 105 Bond Street at Ryerson University. The study involves 2 sessions, one 2.5 - 3 hour session, and one $2-2.5$ hour session. At both sessions, you will be asked to complete some cognitive tasks, and some paper-pencil questionnaires. At the first session, you will also be asked to complete a set of questionnaires that include queries on your demographic information (e.g., age, sex, and native language), education (i.e., the number of years at each educational institution), and personal health (e.g., illness, vision or hearing problems, any previous head injuries, and current medication use). We request this information in order to assess what factors have effects on the experimental tasks you are going to be asked to perform in this study. You may refuse to answer any particular questions or any questionnaires including those pertaining to your health and personal information, without any negative consequences.

Risks and Benefits: Study risk is minimal. The risk, if any, will be no greater than that encountered in everyday life. The stimuli used in this study are commonly encountered in books, newspapers, radio, TV, or movies. Every effort will be taken to ensure that you are as comfortable as possible throughout this research study; however, you may withdraw from the study at any time without any penalty. The major benefit from participating in this study is the opportunity to learn and practice skills in mental processing. You will also contribute to the process of scientific research.

Confidentiality: We will take great care to ensure protection of your confidentiality and privacy. Any information learned about you during this study will be kept confidential, and neither your name nor any other identifying 


\title{
APPENDICES
}

information will be made available to anyone other than the investigators. This information will be secured in a locked filing cabinet at all times. All physical copies of the documents and data will be stored in a secure area and will be shredded when no longer required. In any reports, publications or presentation, no individual will be identified, and only group results will be presented.

Voluntary Nature of Participation: Participation in this study is voluntary. Your choice of whether or not to participate has no bearing on your past, present or future relationship with Ryerson University. If you decide to participate, you are free to withdraw your participation at any time before completion. Since the data will be coded, participants may withdraw up until the time of data analysis. Moreover, you may refuse to answer particular questions, or part of any questionnaires without any penalty.

Compensation: You will be compensated \$10/hour for participation in this study. A prorated amount of compensation is available should you discontinue participation.

Questions about the Study: If you have any questions about the research now, please ask. If you have questions about the research later, you may contact Andrea Wilkinson at (416) 979-5000 (ext.4987). If you have questions regarding your rights as a human subject and participant in this study, you may contact the Ryerson University Research Ethics Board for information.

\author{
Research Ethics Board \\ c/o Office of the Associate Vice President, Academic \\ Ryerson University, 350 Victoria Street \\ Toronto, Ontario, M5B 2K3
}

(416) $979-5042$

Agreement: Your signature below indicates that you have read the information in this agreement and have had the chance to ask any questions you have about the study. Your signature also indicates that you agree to be in the study and have been told that you can change your mind and withdraw your consent to participate at any time.

By signing below, I acknowledge that I have read and understood all of the above statements and agree to voluntarily participate in this study.

Name of Participant (please print)

Signature of Participant

Date

Signature of Investigator

Date 


\title{
APPENDICES
}

\author{
Appendix K \\ Debriefing Form for the Training Group (Chapter 3)
}

\section{DEBRIEFING FORM}

\section{Three Pieces of Inhibition - Practice Study}

Thank you for participating in this experiment! This document explains what we are trying to investigate.

According to previous research, inhibition is a mental ability that has three main functions: access - the ability to keep unimportant information outside your focus of attention, deletion - getting rid of no longer important information, and restraint withholding an urge. Research shows that these functions tend to decline as we get older (Hasher, Zacks, and May, 1999).

The current study aims to determine whether or not we can use practice to boost older adults' performance on all three functions of inhibition (i.e., access, deletion and restraint). In addition, we are interested in learning whether practicing inhibition will help memory performance and speed in processing information. Based on previous studies, we expect that practice on these inhibition tasks will improve performance on them, and will also lead to better memory and mental speed.

The implications of this study are in the development of effective training programs for mental abilities geared towards older adults.

Please do not hesitate to ask the experimenter questions and/or to contact the principle investigator, Andrea Wilkinson, at 416-979-5000 (ext. 4987), if you have any questions or concerns regarding this project. Thank you for your participation!

Hasher, L., Zacks, R. T., \& May, C. P. (1999). Inhibitory control, circadian arousal, and age. In D. Gopher, \& A. Koriat (Eds.), Attention and performance XVII: Cognitive regulation of performance: Interaction of theory and application (pp. 653-675). Cambridge, MA, US: The MIT Press.

Wilkinson, A. J. \& Yang, L. (in press). Plasticity of inhibition in older adults: retest practice and transfer effects. Psychology and Aging. 


\section{APPENDICES}

Appendix L

Debriefing Form for the Control Group (Chapter 3)

\section{DEBRIEFING FORM}

Three Pieces of Inhibition

Thank you for participating in this experiment! This document explains what we are trying to investigate.

According to previous research, inhibition is a mental ability that has three main functions: access - the ability to keep unimportant information outside your focus of attention, deletion - getting rid of no longer important information, and restraint withholding an urge. Research shows that these functions tend to decline as we get older (Hasher, Zacks, and May, 1999).

The current study aims to determine whether or not we can use practice to boost older adults' performance on all three functions of inhibition (i.e., access, deletion and restraint). Some individuals received some additional practice on inhibition. Specifically, we want to determine whether this extra practice adds to performance improvements from session 1 to session 2 . In the individuals who received extra practice, we found that they had better performance at session 2 relative to session 1 on some measures of mental abilities, e.g., speed of processing, and we expect that the group that did not get extra practice will also show improvements, but it may not be as much.

The implications of this study are in the development of effective training programs for mental abilities geared towards older adults.

Please do not hesitate to ask the experimenter questions and/or to contact the principal investigator, Andrea Wilkinson, at 416-979-5000 (ext. 4987), if you have any questions or concerns regarding this project. Thank you for your participation!

Hasher, L., Zacks, R. T., \& May, C. P. (1999). Inhibitory control, circadian arousal, and age. In D. Gopher, \& A. Koriat (Eds.), Attention and performance XVII: Cognitive regulation of performance: Interaction of theory and application (pp. 653-675). Cambridge, MA, US: The MIT Press.

Wilkinson, A. J. \& Yang, L. (in press). Plasticity of inhibition in older adults: retest practice and transfer effects. Psychology and Aging. 


\section{REFERENCES}

\section{References}

Allen, P. A., Weber, T. A., \& Madden, D. J. (1994). Adult age differences in attention: Filtering or selection? Journal of Gerontology, 49, 213-222.

Anand, R., Chapman, S. B., Rackley, A., Keebler, M., Zientz, J., \& Hart Jr., J. (2011). Gist reasoning training in cognitive normal seniors. International Journal of Geriatric Psychiatry, 26, 961-968.

Anderson, M. C. (2001). Active forgetting: Evidence for functional inhibition as a source of memory failure. Journal of Aggression, Maltreatment, \& Trauma, 4, 185-210.

Anderson, N. D. \& Craik, F. I. M. (2000). Memory in the aging brain. In E. Tulving \& F. I. M. Craik (Eds.), The Oxford handbook of memory (pp. 411-425). NY, New York: Oxford University Press, Inc.

Aron, A. R., Robbins, R. W., \& Poldrack, R. A. (2004). Inhibition and the right inferior frontal cortex. Trends in Cognitive Sciences, 8, 170-177.

Bäckman, L., Jones, S., Berger, A.-K., Jonsson Luakka, E., \& Small, B. J. (2005). Cognitive impairment in preclinical Alzheimer's disease: A meta-analysis. Neuropsychology, 19, 520-531.

Badre, D. (2012). Opening the gate to working memory. Proceedings of the National Academy of Sciences of the United States of America, 109, 19878-19879.

Ball, K., Berch, D. B., Helmers, K. F., Jobe, J. B., Leveck, M. D., Marsiske, M., ..., Willis, S. L. (2002). Effects of cognitive training interventions with older adults: A randomized controlled trial. The Journal of the American Medical Association, 288, 2271-2281. 


\section{REFERENCES}

Baltes, P. B., \& Lindenberger, U. (1988). On the range of cognitive plasticity in old age as a function of experience: 15 years of intervention research. Behavior Therapy, 19, 283-300.

Baltes, P. B., Sowarka, D., \& Kliegl, R. (1989). Cognitive training research on fluid intelligence in old age: What can older adults achieve by themselves? Psychology and Aging, 4, 217221.

Bartzokis, G., Cummings, J. L., Sultzer, D., Henderson, V. W., Nuechterlein, K. H., \& Mintz, J. (2003). White matter structural integrity in healthy aging adults and patients with Alzheimer Disease: A magnetic resonance imaging study. Archives of Neurology, 60, 393398.

Beck, A. T., Epstein, N., Brown, G., \& Steer, R. A. (1988). An inventory for measuring clinical anxiety: Psychometric properties. Journal of Consulting and Clinical Psychology, 56, 893897. doi:10.1037/0022-006X.56.6.893

Bherer, L., Kramer, A. F., Peterson, M. S., Colcombe, S., Erickson, K., \& Becic, E. (2005). Training effects on dual-task performance: Are there age-related differences in plasticity of attentional control? Psychology and Aging, 20, 695-709.

Bjorklund, D. F. \& Kipp Harnishfeger, K. (1990). The resources construct in cognitive development: Diverse sources of evidence and a theory of inefficient inhibition. Developmental Review, 10, 48-71.

Blieszner, R., Willis, S. L., \& Baltes, P. B. (1981). Training research in aging on the fluid ability of inductive reasoning. Journal of Applied Developmental Psychology, 2, 247-265. doi:10.1016/0193-3973(81)90005-8

Borella, E., Carretti, B., Riboldi, F., \& De Beni, R. (2010). Working memory training in older adults: Evidence of transfer and maintenance effects. Psychology and Aging, 25, 767-778. 


\section{REFERENCES}

Bradley, M. M., \& Lang, P. J. (1999). Affective norms for English words (ANEW). Gainesville, FL: University of Florida, NIMH Center for the Study of Emotion and Attention.

Braver, T. S., Barch, D. M., Keys, B. A., Carter, C. S., Cohen, J. D., Kaye, J. A., . ., Reed, B. R. (2001). Context processing in older adults: Evidence for a theory relating cognitive control to neurobiology in healthy aging. Journal of Experimental Psychology: General, 130, 746763.

Braver, T. S. \& Cohen, J. D. (2000). On the control of control: The role of dopamine in regulating prefrontal function and working memory. In S. Monsell \& J. Driver (Eds.), Control of cognitive processes (pp. 713-737). Cambridge, MA: The MIT Press.

Braver, T.D., Cohen, J.D., Nystrom, L.E., Jonides, J., Smith, E.E., \& Noll, D.C. (1997). A parametric study of prefrontal cortex involvement in human working memory. NeuroImage, 5, 49-62.

Brehmer, Y., Westerberg, H., \& Bäckman, L. (2012). Working-memory training in younger and older adults: Training gains, transfer and maintenance. Frontiers in Human Neuroscience, $6,1-7$.

Brookmeyer, R., Johnson, E., Ziegler-Graham, K., \& Arrighi, H. M. (2007). Forecasting the global burden of Alzheimer’s disease. Alzheimer's \& Dementia, 3, 186-191.

Buitenweg, J. I. V., Murre, J. M. J., \& Ridderinkhof, K. R. (2012). Brain training in progress: A review of trainability in healthy seniors. Frontiers in Human Neuroscience, 6, 1-11.

Cabeza, R. \& Dennis, N. A. (2013). Frontal lobes and aging: Deterioration and compensation. In D. T. Stuss \& R. T. Knight (Eds.). Principles of frontal lobe function, 2nd Edition (pp. 628-652). New York, NY: Oxford University Press. 


\section{REFERENCES}

Case, R. (1985). Intellectual development: Birth to adulthood. New York: Academic Press.

Case, R., Kurland, M., \& Goldberg, J. (1982). Operational efficiency and the growth of shortterm memory span. Journal of Experimental Child Psychology, 33, 386-404.

Casey, B. J., Trainor, R. J., Orendi, J. L., Schubert, A. B., Nystrom, L. E., Giedd, J. N., . . ., Rapoport, J. L. (1997). A developmental functional MRI study of prefrontal activation during performance of a Go-No-Go task. Journal of Cognitive Neuroscience, 9, 835-847.

Chikazoe, J., Jimura, K., Asari, T., Yamashita, K., Morimoto, H., Hirose, S., . . ., Konishi, S. (2009). Functional dissociation in right inferior frontal cortex during performance of Go/No-Go task. Cerebral Cortex, 19, 146-152.

Clay, O. J., Wadley, V. G., Edwards, J. D., Roth, D. L., Roenker, D. L., \& Ball, K. K. (2005). Cumulative meta-analysis of the relationship between useful field of view and driving performance in older adults: Current and future implications. Optometry and Vision Science, 82, 724-731.

Cohen, J. (1988). Statistical power analysis for the behavioral sciences, $2^{\text {nd }}$ Edition. Hillsdale, NJ: Lawrence Erlbaum Associates, Inc.

Cohen, J. (1992). A power primer. Psychological Bulletin, 112, 155-159.

Colcombe, S. \& Kramer, A. F. (2003). Fitness effects on the cognitive function of older adults: A meta-analytic study. Psychological Science, 14, 125-130.

Connelly, S. L., Hasher, L., \& Zacks, R. T. (1991). Age and reading: The impact of distraction. Psychology and Aging, 6, 533-541.

Conway, M. A. \& Fthenaki, A. (2003). Disruption of inhibitory control of memory following lesions to the frontal and temporal lobes. Cortex, 39, 667-686. 


\section{REFERENCES}

Corsi, P. M. (1972). Human memory and the medial temporal region of the brain. Dissertation Abstracts International, 34 (02), 819B. (University Microfilms No. AA105-77717).

Craik, F. I. M. (1983). On the transfer of information from temporary to permanent memory. Philosophical Transactions of the Royal Society of London, B 302, 341-359.

Craik, F. I. M. (1986). A functional account of age differences in memory. In F. Klix \& Hagendorf H. (Eds.), Human memory and cognitive capabilities: Mechanisms and performances (pp. 409-422). Amsterdam, North-Holland: Elsevier Science Publishing Company.

Craik, F. I. M. \& Byrd, M. (1982). Aging and cognitive deficits: The role of attentional resources. In Craik F. I. M. \& Trehub S. E. (Eds.), Aging and cognitive processes (pp. 191211). New York, NY: Plenum Press.

Craik, F. I. M. \& Jennings, J. M. (2000). Human memory. In F. I. M. Craik \& T. A. Salthouse (Eds.), The handbook of aging and cognition (pp.51-110). Hillsdale, NJ: Lawrence Erlbaum Associates, Inc.

Dahlin, E., Nyberg, L., Bäckman, L., \& Stigsdotter-Neely, A. (2008a). Plasticity of executive functioning in young and older adults: Immediate training gains, transfer, and long-term maintenance. Psychology and Aging, 23, 720-730.

Dahlin, E., Stigsdotter-Neely, A., Larsson, A., Bäckman, L. \& Nyberg, L. (2008b). Transfer of learning after updating training mediated by the striatum. Science, 320, 1510-1512.

D’Ardenne, K., Eshel, N., Luka, J., Lenartowicz, A., Nystrom, L. E., \& Cohen, J. D. (2012). Role of prefrontal cortex and the midbrain dopamine system in working memory updating. Proceedings of the National Academy of Sciences, 109, 19900-19909. 


\section{REFERENCES}

Davidson, D. J., Zacks, R. T., \& Williams, C. C. (2003). Stroop interference, practice, and aging. Aging, Neuropsychology, and Cognition, 10, 85-98. doi:10.1076/anec.10.2.85.14463

DeCarli, C., Murphy, D. G., Gillette, J. A., Haxby, J. V., Teichberg, D., Schapiro, M. B., \& Horwitz, B. (1994). Lack of age-related differences in temporal lobe volume of very healthy adults. American Journal of Neuroradiology, 15, 689-696.

Delis, D. C., Roberston, L. C., \& Efron, R. (1986). Hemispheric specialization of memory for visual hierarchical stimuli. Neuropsychologia, 24, 205-214.

Dempster, F. N. (1992). The rise and fall of the inhibitory mechanism: Toward a unified theory of cognitive development and aging. Developmental Review, 12, 45-75.

Dempster, F. N. (1993). Resistance to interference. Developmental changes in a basic processing dimension. In M. L. Howe \& R. Pasnak (Eds.), Emerging themes in cognitive development. Vol. 1: Foundations (pp.3-27). New York: Springer-Verlag.

Dennis, M. (2006). Prefrontal cortex: Typical and atypical development. In J. Risberg \& J. Grafman (Eds.), The frontal lobes: Development, function and pathology (pp. 128-162). United Kingdom: Cambridge University Press.

Dobbs, A. R. \& Rule, B. G. (1989). Adult age differences in working memory. Psychology and Aging, 4, 500-503.

Dolcos, F., Rice, H. J., \& Cabeza, R. (2002). Hemispheric asymmetry and aging: Right hemisphere decline or asymmetry reduction. Neuroscience and Biobehavioral Reviews, 26, 819-825.

Donders, F. C. (1969). On the speed of mental processes. In W. G. Koster (Ed.), Attention and performance II (pp. 412-431). Amsterdam: North-Holland Publishing Co. (Original work published in 1868). 


\section{REFERENCES}

Dulaney, C. L., \& Rogers, W. A. (1994). Mechanisms underlying reduction in Stroop interference with practice for young and old adults. Journal of Experimental Psychology: Learning, Memory, and Cognition, 20, 470-484. doi:10.1037/0278-7393.20.2.470

Dunlap, W. P., Cortina, J. M., Vaslow, J. B., \& Burke, M. J. (1996). Meta-analysis of experiments with matched groups or repeated measures designs. Psychological Methods, 1, $170-177$.

Dvorine Pseudo-Isochromatic Plates (2 ${ }^{\text {nd }}$ edition) [Apparatus]. (1953). Baltimore, United States: Harcourt, Brace \& World, Inc.

Edwards, J. D., Wadley, V. G., Vance, D. E., Wood, K., Roenker, D. L., \& Ball, K. K. (2005). The impact of speed of processing training on cognitive and everyday performance. Aging \& Mental Health, 9, 262-271.

English, H. B., \& English, A. C. (1958). A comprehensive dictionary of psychological and psychoanalytic terms: A guide to usage. New York: Longmans, Green \& Co., Inc.

Erixon-Lindroth, N., Farde, L., Wahlin, T.-B. R., Sovago, J., Halldin, C., \& Bäckman, L. (2005). The role of the striatal dopamine transporter in cognitive aging. Psychiatry Research: Neuroimaging, 138, 1-12.

Falkenstein, M., Hoormann, J., \& Hohnsbein, J. (2002). Inhibition-related ERP components: Variation with modality, age, and time-on-task. Journal of Psychophysiology, 16, 167-175.

Fan, J., McCandliss, B. D., Sommer, T., Raz, A., \& Posner, M. I. (2002). Testing the efficiency and independence of attentional networks. Journal of Cognitive Neuroscience, 14, 340-347. doi:10.1162/089892902317361886 


\section{REFERENCES}

Faul, F., Erdfelder, E., Lang, A.-G., \& Buchner, A. (2007). G*Power 3: A flexible statistical power analysis program for the social, behavioral, and biomedical sciences. Behavior Research Methods, 39, 175-191.

Feyereisen, P. \& Charlot, V. (2008). Are there uniform age-related changes across tasks involving inhibitory control through access, deletion, and restraint functions? A preliminary investigation. Experimental Aging Research, 34, 392-418.

Fink, G. R., Halligan, P. W., Marshall, J. C., Frith, C. D., Frackowiak, R. S. J., \& Dolan, R. J. (1997). Neural mechanisms involved in the processing of global and local aspects of hierarchically organized visual stimuli. Brain, 120, 1779-1791.

Fjell, A. M., Walhovd, K. B., Fennema-Notestine, C., McEvoy, L. K., Hagler, D., Holland, D., . . ., Dale, A. M. (2009). One-year brain atrophy evident in healthy aging. Journal of Neuroscience, 29, 15223-15231.

Friedman, N. P. \& Miyake, A. (2004). The relations among inhibition and interference control functions: A latent-variable analysis. Journal of Experimental Psychology: General, 133, 101-135.

Garavan, H. Ross, T. J., \& Stein, E. A. (1999). Right hemispheric dominance of inhibitory control: An event-related functional MRI study. Proceedings of the National Academy of Sciences, 96, 8301-8306.

Gazzaley, A., Cooney, J. W., Rissman, J., \& D'Esposito, M. (2005). Top-down suppression deficit underlies working memory impairment in normal aging. Nature Neuroscience, 8, 1298-1300. doi:10.1038/nn1543 


\section{REFERENCES}

Gazzaley, A., Rissman, J., Cooney, J., Rutman, A., Seibert, T., Clapp, W., \& D’Esposito, M. (2007). Functional interactions between prefrontal and visual association cortex contribute to top-down modulation of visual processing. Cerebral Cortex, 17, 125-135.

Gick, M. L., Craik, F. I. M., \& Morris, R. G. (1988). Task complexity and age differences in working memory. Memory \& Cognition, 16, 353-361.

Grafman, J. (2006). Human prefrontal cortex: processes and representations. In J. Risberg \& J. Grafman (Eds.), The frontal lobes: Development, function and pathology (pp. 69-91). United Kingdom: Cambridge University Press.

Günther, V. K., Schäfer, P., Holzner, J., \& Kemmler, G. W. (2003). Long-term improvements in cognitive performance through computer-assisted cognitive training: a pilot study in a residential home for older people. Aging \& Mental Health, 7, 200-206. doi: 10.1080/1360786031000101175

Hasher, L., Lustig, C., \& Zacks, R. (2007). Inhibitory mechanisms and the control of attention. In A. R. A. Conway, C. Jarrold, M. J. Kane, A. Miyake \& J. N. Towse (Eds.), Variation in working memory (pp. 227-249). New York, NY, US: Oxford University Press.

Hasher, L., Tonev, S. T., Lustig, C., \& Zacks, R. T. (2001). Inhibitory control, environmental support, and self-initiated processing in aging. In M. Naveh-Benjamin, M. Moscovitch, \& R. L. Roediger, III (Eds.), Perspectives on human memory and cognitive aging: Essays in honour of Fergus Craik (pp. 286-297). East Sussex, UK: Psychology Press.

Hasher, L. \& Zacks, R. T. (1979). Automatic and effortful processes in memory. Journal of Experimental Psychology: General, 108, 356-388. 


\section{REFERENCES}

Hasher, L., \& Zacks, R. T. (1988). Working memory, comprehension, and aging: A review and new view. In G.H. Bower (Ed.), The psychology of learning and motivation (Vol. 22, pp.193-225). New York: Academic Press.

Hasher, L., Zacks, R. T., \& May, C. P. (1999). Inhibitory control, circadian arousal, and age. In D. Gopher, \& A. Koriat (Eds.), Attention and performance XVII: Cognitive regulation of performance: Interaction of theory and application (pp. 653-675). Cambridge, MA, US: The MIT Press.

Head, D., Buckner, R. L., Shimony, J. S., Williams, L. E., Akbudak, E., Conturo, T. E., ...., Snyder, A. Z. (2004). Differential vulnerability of anterior white matter in nondemented aging with minimal acceleration in dementia of the Alzheimer type: Evidence from diffusion tensor imaging. Cerebral Cortex, 14, 410-423.

Hedden, T. (2007). Imaging cognition in the aging human brain. In D. R. Riddle (Ed.), Brain aging: Models, methods, and mechanisms (pp.251-276). Boca Raton, FL: Taylor \& Francis Group, LLC.

Hedden, T. \& Gabrieli, J. D. E. (2004). Insights into the ageing mind: A view from cognitive neuroscience. Nature Reviews Neuroscience, 5, 87 - 96.

Heilman, K. M. \& Van Den Abell, T. (1980). Right hemisphere dominance for attention: The mechanism underlying hemispheric asymmetries of inattention (neglect). Neurology, 30, 327-330. 


\section{REFERENCES}

Houghton, G., \& Tipper, S. P. (1994). A model of inhibitory mechanisms in selective attention.

In D. Dagenbach \& T. H. Carr (Eds.), Inhibitory processes in attention, memory, and language (pp. 53-112). San Diego, CA: Academic Press, Inc.

Jack, C. R., Jr., Petersen, R. C., Xu, Y., O’Brien, P. C., Smith, G. E., Ivnik, R. J., . . , Kokmen, E. (1998). Rate of medial temporal lobe atrophy in typical aging and Alzheimer's disease. Neurology, 51, 993-999.

Jonides, J., Badre, D., Curtis, C., Thompson-Schill, S. L., \& Smith, E. E. (2002). Mechanisms of conflict resolution in prefrontal cortex. In D. T. Stuss \& R. T. Knight (Eds.), Principles of frontal lobe function (pp. 233-245). New York, NY: Oxford University Press, Inc.

Karbach, J. \& Kray, J. (2009). How useful is executive control training? Age differences in near and far transfer of task-switching training. Developmental Science, 12, 978-990. doi: 10.1111/j.1467-7687.2009.00846.x

Katzman, R., Brown, T., Fuld, P., Peck, A., Schechter, R., \& Schimmel, H. (1983). Validation of a short orientation-memory-concentration test of cognitive impairment. The American Journal of Psychiatry, 140, 734-739.

Kemper, T. L. (1994). Neuroanatomical and neuropathological changes during aging and in dementia. In M. L. Albert \& J. E. Knoefel (Eds.), Clinical neurology of aging, $2^{\text {nd }}$ Edition (pp. 3-67). New York, NY: Oxford University Press, Inc.

Kipp Harnishfeger, K. (1995). The development of cognitive inhibition: Theories, definitions, and research evidence. In F. N. Dempster \& C. J. Brainerd (Eds.), Interference and inhibition in cognition (pp. 175-204). San Diego, CA: Academic Press. 


\section{REFERENCES}

Kipp Harnishfeger, K. \& Bjorklund, D. F. (1993). The ontogeny of inhibition mechanisms: A renewed approach to cognitive development. In M. L. Howe \& R. Pasnak (Eds.), Emerging themes in cognitive development. Vol. 1: Foundations (pp.3-27). New York: SpringerVerlag.

Kirchner, W. K. (1958). Age differences in short-term retention of rapidly changing information. Journal of Experimental Psychology, 55, 352-358.

Kotchoubey, B., Wascher, E., \& Verleger, R. (1997). Shifting attention between global features and small details: An event-related potential study. Biological Psychology, 46, 25-50.

Kramer, A. F., Hahn, S., \& Gopher, D. (1999). Task coordination and aging: Explorations of executive control processes in the task switching paradigm. Acta Psychologica, 101, 339378.

Leung, H.-C., Skudlarski, P., Gatenby, J. C., Peterson, B. S., \& Gore, J. C. (2000). An eventrelated functional MRI study of the Stroop colour word interference task. Cerebral Cortex, 10, 552-560.

Li, S.-C., Schmiedek, F., Huxhold, O., Röcke, C., Smith, J., \& Lindenberger, U. (2008). Working memory plasticity in old age: Practice gain, transfer, and maintenance. Psychology and Aging, 23, 731-742.

Little, D. M., \& Hartley, A. A. (2000). Further evidence that negative priming in the Stroop color-word task is equivalent in older and younger adults. Psychology and Aging, 15, 9-17. doi:10.1037/0882-7974.15.1.9

Lustig, C., Hasher, L., \& Tonev, S. T. (2006). Distraction as a determinant of processing speed. Psychonomic Bulletin \& Review, 13, 619-625. 


\section{REFERENCES}

MacLeod, C. M. (1991). Half a century of research on the Stroop effect: An integrative review. Psychological Bulletin, 109, 163-203. doi:10.1037/0033-2909.109.2.163

MacLeod, C. M. (1998). Directed forgetting. In J. M. Golding \& C. M. MacLeod (Eds.), Intentional forgetting: Interdisciplinary approaches (pp.1-57). Mahwah, New Jersey: Lawrence Erlbaum Associates, Publishers.

MacLeod, C. M. (1999). The item and list methods of directed forgetting: Test differences and the role of demand characteristics. Psychonomic Bulletin \& Review, 6, 123-129.

MacLeod, C. M., Dodd, M. D., Sheard, E. D., Wilson, D. E., \& Bibi, U. (2003). In opposition to inhibition. In G.H. Bower (Ed.), The psychology of learning and motivation (Vol. 43, pp.163-214). New York: Academic Press.

MacLeod, C. M., \& MacDonald, P. A. (2000). Interdimensional interference in the Stroop effect: Uncovering the cognitive and neural anatomy of attention. Trends in Cognitive Sciences, 4, 383-391.

Mahncke, H. W., Connor, B. B., Appelman, J., Ahsanuddin, O. N., Hardy, J. L., Wood, R. A., . . ., Merzenich, M. M. (2006). Memory enhancement in healthy older adults using a brain plasticity-based training program: A randomized, controlled study. Proceedings of the National Academy of Sciences of the United States of America, 103, 12523-12528.

May, C. P., Hasher, L., \& Kane, M. J. (1999). The role of interference in memory span. Memory \& Cognition, 27, 759-767.

McAvinue, L. P., Habekost, T., Johnson, K. A., Kyllingsbaek, S., Vangkilde, S., Bundesen, C., \& Robertson, I. H. (2012). Sustained attention, attentional selectivity, and attentional capacity across the lifespan. Attention, Perception, \& Psychophysics, 74, 1570-1582. 


\section{REFERENCES}

Milham, M. P., Erickson, K. I., Banich, M. T., Kramer, A. F., Webb, A., Wszalek, T., \& Cohen, N. J. (2002). Attentional control in the aging brain: Insights from an fMRI study of the Stroop task. Brain and Cognition, 49, 277-296.

Miller, E. K. \& Cohen, J. D. (2001). An integrative theory of prefrontal cortex function. Annual Review of Neuroscience, 24, 167-202.

Miyake, A., Friedman, N. P., Emerson, M. J., Witzki, A. H., Howerter, A., \& Wager, T. D. (2000). The unity and diversity of executive functions and their contributions to complex “frontal lobe” tasks: A latent variable analysis. Cognitive Psychology, 41, 49-100.

Mottaghy, F. M., Krause, B. J., Kemna, L. J., Töpper, R., Tellman, L., Beu, M., . . ., MüllerGärtner, H.-W. (2000). Modulation of the neuronal circuitry subserving working memory in healthy human subjects by repetitive transcranial magnetic stimulation. Neuroscience Letters, 280, 167-170.

Navon, D. (1977). Forest before trees: The precedence of global features in visual perception. Cognitive Psychology, 9, 353-383.

Nielson, K. A., Langenecker, S. A., \& Garavan, H. (2002). Differences in the functional neuroanatomy of inhibitory control across the adult life span. Psychology and Aging, 17, 5671.

Nigg, J. T. (2000). On inhibition/disinhibition in developmental in development psychopathology: Views from cognitive and personality psychology and a working inhibition taxonomy. Psychological Bulletin, 126, 220-246.

Owen, A. M., McMillan, K. M., Laird, A. R., \& Bullmore, E. (2005). N-Back working memory paradigm: A meta-analysis of normative functional neuroimaging studies. Human Brain Mapping, 25, 46-59. 


\section{REFERENCES}

Paus, T., Jech, R., Thompson, C. J., Comeau, R., Peters, T., \& Evans, A. C. (1997). Transcranial magnetic stimulation during positron emission tomography: A new method of studying connectivity of the human cerebral cortex. The Journal of Neuroscience, 17, 3178-3184.

Pfefferbaum, A., Adalsteinsson, E., \& Sullivan, E. V. (2005). Frontal circuitry degradation marks healthy adult aging: Evidence from diffusion tensor imaging. NeuroImage, 26, 891-899.

Radloff, L. S. (1977). The CES-D scale: A self-report depression scale for research in the general population. Applied Psychological Measurement, 1, 385-401.

Raz, N. (2000). Aging of the brain and its impact on cognitive performance: Integration of structural and functional findings. In F. I. M. Craik \& T. A. Salthouse (Eds.), The handbook of aging and cognition (2 ${ }^{\text {nd }}$ ed.) (pp.1-90). Mahwah, NJ: Lawrence Erlbaum Associates, Inc.

Raz, N. (2005). The aging brain observed in vivo: Differential changes and their modifiers. In R. Cabeza, L. Nyberg, \& D. Park (Eds.), Cognitive neuroscience of aging: Linking cognitive and cerebral aging (pp. 19-57.) New York, NY: Oxford University Press, Inc.

Raz, N., Gunning, F. M., Head, D., Dupuis, J. H., McQuain, J., Briggs, S. D., . ., Acker, J. D. (1997). Selective aging of the human cerebral cortex observed in vivo: Differential vulnerability of the prefrontal gray matter. Cerebral Cortex, 7, 268-282.

Resnick, S. M., Pham, D. L., Kraut, M. A., Zonderman, A. B., \& Davatzikos, C. (2003). Longitudinal magnetic resonance imaging studies of older adults: A shrinking brain. The Journal of Neuroscience, 23, 3295-3301.

Rizio, A. A. \& Dennis, N. A. (2013). The neural correlates of cognitive control: Successful remembering and intentional forgetting. Journal of Cognitive Neuroscience, 25, 297-312.

Robertson, L. C. \& Lamb, M. R. (1991). Neuropsychological contributions to theories of part/whole organization. Cognitive Psychology, 23, 299-330. 


\section{REFERENCES}

Robertson, I. H., Manly, T., Andrade, J., Baddeley, B. T., \& Yiend, J. (1997). 'Oops!’: Performance correlates of everyday attentional failures in traumatic brain injured and normal subjects. Neuropsychologia, 35, 747-758.

Rosenbaum Pocket Vision Screener [Apparatus]. (1986). Canada: Merck Sharp \& Dohme.

Rowe, G., Hasher, L., \& Turcotte, J. (2009). Age and synchrony effects in visuospatial working memory. The Quarterly Journal of Experimental Psychology, 62, 1873-1880. doi:10.1080/17470210902834852

Rubia, K., Russell, T., Overmeyer, S., Brammer, M. J., Bullmore, E. T., Sharma, T., . . T Taylor, E. (2001). Mapping motor inhibition: Conjunctive brain activations across different versions of Go/No-Go and Stop Tasks. NeuroImage, 13, 250-261.

Salat, D. H., Kaye, J. A. \& Janowsky, J. S. (1999). Prefrontal gray and white matter volumes in healthy aging and Alzheimer disease. Archives of Neurology, 56, 338-344.

Salat, D. H., Kaye, J. A. \& Janowsky, J. S. (2001). Selective preservation and degeneration within the prefrontal cortex in aging and Alzheimer disease. Archives of Neurology, 58, 1403-1408.

Salat, D. H., Tuch, D. S., Greve, D. N., van der Kouwe, A. J. W., Hevelone, N. D., Zaleta, A. K., ..., Dale, A. M. (2005). Age-related alterations in white matter microstructure measured by diffusion tensor imaging. Neurobiology of Aging, 26, 1215-1227.

Salthouse, T. A. \& Babcock, R. L. (1991). Decomposing adult age differences in working memory. Developmental Psychology, 27, 763-776.

Schneider, W. \& Shiffrin, R. M. (1977). Controlled and automatic human information processing: I. Detection, Search, and Attention. Psychological Review, 84, 1-66. 


\section{REFERENCES}

Sego, S. A., Golding, J. M., \& Gottlob, L. R. (2006). Directed forgetting in older adults using item and list methods. Aging, Neuropsychology, and Cognition, 13, 1-20.

Slavin, M.J., Mattingley, L.B., Bradshaw, J.L., \& Storey, E. (2002). Local-global processing in Alzheimer's disease: an examination of interference, inhibition and priming. Neuropsychologia, 40, 1173-1186.

Smith, G. E., Housen, P., Yaffe, K., Ruff, R., Kennison, R. F., Mahncke, H. W., \& Zelinski, E. M. (2009). A cognitive training program based on principles of brain plasticity: Results from the Improvement in Memory with Plasticity-based Adaptive Cognitive Training (IMPACT) study. Journal of the American Geriatrics Society, 57, 594-603.

Snodgrass, J. G. \& Corwin, J. (1988). Pragmatics of measuring recognition memory: Applications to dementia and amnesia. Journal of Experimental Psychology: General, 117, $34-50$.

Stigsdotter-Neely, A., \& Bäckman, L. (1993). Long-term maintenance of gains from memory training in older adults: Two 31ำ-year follow-up studies. Journal of Gerontology, 48, 233237.

Stroop, J. R. (1935). Studies of interference in serial verbal reactions. Journal of Experimental Psychology, 18, 643-662. doi:10.1037/h0054651

Suhara, T., Fukuda, H., Inoue, O., Itoh, T., Suzuki, K., Yamasaki, T., \& Tateno, Y. (1991). Agerelated changes in human D1 dopamine receptors measured by positron emission tomography. Psychopharmacology, 103, 41-45.

Thomas, R. C., Kim, S., Goldstein, D., Hasher, L., Wong, K., \& Ghai, A. (2007). Do you see what I see? The impact of age differences in time perspective on visual attention. Journal of Gerontology: Psychological Sciences, 62B, 247-252. 


\section{REFERENCES}

Thompson, K. M., Fawcett, J. M., \& Taylor, T. L. (2011). Tag, you're it: Tagging as an alternative to yes/no recognition in item method directed forgetting. Acta Psychologica, 138, 171-175.

Thompson, G., \& Foth, D. (2005). Cognitive-training programs for older adults: What are they and can they enhance mental fitness? Educational Gerontology, 31, 603-626.

Thorndike, E. L. \& Woodworth, R. S. (1901). The influence of improvement in one mental function upon the efficiency of other functions. Psychological Review, 8, 247-261.

Titz, C. \& Verhaeghen, P. (2010). Aging and directed forgetting in episodic memory: A metaanalysis. Psychology and Aging, 25, 405-411.

Ullsperger, M., Mecklinger, A. \& Müller, U. (2000). An electrophysiological test of directed forgetting: The role of retrieval inhibition. Journal of Cognitive Neuroscience, 12, 924940.

van Muijden, J., Band, G. P H., \& Hommel, B. (2012). Online games training aging brains: Limited transfer to cognitive control functions. Frontiers in Human Neuroscience, 6, 1-13.

Verhaeghen, P. \& Basak, C. (2005). Ageing and switching of the focus of attention in working memory: Results from a modified N-Back task. The Quarterly Journal of Experimental Psychology, 58A, 134-154.

Volkow, N. D., Gur, R. C., Wang, G.-J., Fowler, J. S., Moberg, P. J., Ding, Y.-S., . ., Logan, J. (1998). Association between decline in brain dopamine activity with age and cognitive and 


\section{REFERENCES}

motor impairment in healthy individuals. The American Journal of Psychiatry, 155, 344349.

Warriner, A. B., Kuperman, V., \& Brysbaert, M. (2013). Norms of valence, arousal, and dominance for 13,915 English lemmas. Behavior Research Methods. Advance online publication. doi: 10.3758/s13428-012-0314-X

Wechsler, D. (1981). WAIS-R manual. New York: Psychological Corporation.

West, R. L. (1996). An application of prefrontal cortex function theory to cognitive aging. Psychological Bulletin, 120, 272-292.

West, R., \& Alain, C. (2000). Age-related decline in inhibitory control contributes to the increased Stroop effect observed in older adults. Psychophysiology, 37, 179-189.

Wilkinson, A. J., \& Yang, L. (2012). Plasticity of inhibition in older adults: Retest practice and transfer effects. Psychology and Aging, 27, 606-615. doi: 10.1037/a0025926

Willis, S. L., Blieszner, R. \& Baltes, P. B. (1981). Intellectual training research in aging: Modification of performance on the fluid ability of figural relations. Journal of Educational Psychology, 73, 41-50.

Willis, S. L., \& Nesselroade, C. S. (1990). Long-term effects of fluid ability training in old-old age. Developmental Psychology, 26, 905-910. doi: 10.1037/0012-1649.26.6.905

Willis, S. L., Tennstedt, S. L., Marsiske, M., Ball, K., Elias, J., Mann Koepke, K., ..., Wright, E. (2006). Long-term effects of cognitive training on everyday functional outcomes in older adults. Journal of American Medical Association, 296, 2805-2814. doi:10.1001/jama.296.23.2805 


\section{REFERENCES}

Wilson, S. P. \& Kipp, K. (1998). The development of efficient inhibition: Evidence from directed-forgetting tasks. Developmental Review, 18, 86-123.

Wylie, G. R., Foxe, J. J., \& Taylor, T. L. (2008). Forgetting as an active process: An fMRI investigation of item-method-directed forgetting. Cerebral Cortex, 18, 670-682.

Yang, L, \& Krampe, R. T. (2009). Long-term maintenance of retest learning in young old and oldest old adults. Journal of Gerontology: Psychological Sciences, 64B, 608-611. doi: 10.1093/geronb/gbp063

Yang, L., Krampe, R. T., \& Baltes, P. B. (2006). Basic forms of cognitive plasticity extended into the oldest-old: Retest learning, age, and cognitive functioning. Psychology and Aging, 21, 372-378.

Yang, L., Reed, M., Russo, F. A., \& Wilkinson, A. (2009a). A new look at retest learning in older adults: Learning in the absence of item-specific effects. The Journals of Gerontology: Psychological Sciences and Social Sciences, 64B, 470-473.

Yang, L., Reed, M., \& Wilkinson, A. (2009b). Memory training in oldest old and young old adults using Method-of-Loci with self-generated loci. APA $117^{\text {th }}$ Annual Convention, August 6-9, Toronto, Ontario.

Zacks, R. T., Radvansky, G., \& Hasher, L. (1996). Studies of directed forgetting in older adults. Journal of Experimental Psychology: Learning, Memory, and Cognition, 22, 143-156.

Zeidan, F., Johnson, S. K., Diamond, B. J., David, Z., \& Goolkasian, P. (2010). Mindfulness meditation improves cognition: Evidence of brief mental training. Consciousness and Cognition, 19, 597-605. 